\title{
Passive separation control of a NACA0012 airfoil via a flexible flap
}

DOI:

$10.1063 / 1.5118933$

Document Version

Accepted author manuscript

Link to publication record in Manchester Research Explorer

\section{Citation for published version (APA):}

Fang, Z., Gong, C., Revell, A., Chen, G., Harwood, A., \& O'connor, J. (2019). Passive separation control of a NACA0012 airfoil via a flexible flap. Physics of Fluids, 31(10), 101904. https://doi.org/10.1063/1.5118933

\section{Published in:}

Physics of Fluids

\section{Citing this paper}

Please note that where the full-text provided on Manchester Research Explorer is the Author Accepted Manuscript or Proof version this may differ from the final Published version. If citing, it is advised that you check and use the publisher's definitive version.

\section{General rights}

Copyright and moral rights for the publications made accessible in the Research Explorer are retained by the authors and/or other copyright owners and it is a condition of accessing publications that users recognise and abide by the legal requirements associated with these rights.

\section{Takedown policy}

If you believe that this document breaches copyright please refer to the University of Manchester's Takedown Procedures [http://man.ac.uk/04Y6Bo] or contact uml.scholarlycommunications@manchester.ac.uk providing relevant details, so we can investigate your claim.

\section{OPEN ACCESS}




\title{
Passive Separation Control of a NACA0012 Airfoil via a Flexible Flap
}

\author{
Zhe Fang(方哲) ${ }^{\text {a) }}$, Chunlin Gong(龚春林) ${ }^{\text {a) }}$, Alistair Revell ${ }^{\text {b) }}$, Gang Chen(陈刚) ${ }^{\text {c) }}$, Adrian Harwood ${ }^{\text {b) }}$, Joseph \\ O’Connor b) \\ a) Shaanxi Aerospace Flight Vehicle Design Key Laboratory, School of Astronautics, Northwestern Polytechnical \\ University, Xi'an, Shaanxi, China \\ ${ }^{b)}$ School of Mechanical, Aerospace and Civil Engineering, University of Manchester, UK \\ ${ }^{c}$ State Key Laboratory for Strength and Vibration of Mechanical Structures, School of Aerospace, Xian Jiaotong \\ University, Xi'an, Shaanxi, China \\ leonwood@nwpu.edu.cn fangzhe@mail.nwpu.edu.cn alistair.revell@manchester.ac.uk aachengang@xjtu.edu.cn \\ adrian.harwood@manchester.ac.uk joseph.oconnor@manchester.ac.uk
}

\begin{abstract}
.
The incorporation of nature-inspired techniques to control or reduce boundary layer separation, to bring about performance enhancements on air/water vehicles, has been an active research area for many years. In this paper a baseline NACA0012 airfoil is modified using a short flap on its upper surface at Reynolds number $R e=1000$. The impact of the flap configuration - described by length, attachment position, deployment angle and material properties, on the aerodynamic performance of the airfoil - quantified by mean and fluctuating forces, is investigated and the flow field is analysed. Inspired by the observation of pop-up feathers on a bird's wing, the flap is first set to be rigid for a range of location, size and inclination angles. After the optimal location of a rigid flap has been established, the flap is then allowed to be flexible, its motion coupled to the encircling flow field, and it is tested for a range of mass ratios and bending stiffness values. The fluid motion is obtained by solving the lattice Boltzmann equation while the dynamics of the flexible flap are calculated using the finite element method (FEM) and the coupling between flow and flap handled by the immersed boundary method (IBM). For the flexible flap, two flapping patterns are observed and the mechanism of separation control via rigid/flexible flap is explained. Compared to the flapless NACA0012 airfoil case, the case with a flap of optimal configuration, the mean lift coefficient is improved by $13.51 \%$, the mean drag coefficient is decreased by $3.67 \%$, the mean lift-drag ratio is improved by $17.84 \%$, the maximum lift fluctuation is decreased by $40.90 \%$ and the maximum drag fluctuation is decreased by $56.90 \%$.
\end{abstract}

Key words: Passive separation control, flap, lattice Boltzmann, immersed boundary, finite element

\section{Introduction}

Due to their high flight efficiency, high manoeuverability and low flight noise, micro aerial vehicles (MAVs) are becoming more and more popular in recent decades and have been applied in many areas, such as environmental protection, traffic monitoring, earthquake search/rescue and movie photography. On most occasions, these MAVs have to operate in closed, narrow environments, which dictates their small sizes and must be capable of flying at low speeds. Yet they need to be agile enough to move around sharp corners and bends [1]. These operational limits cause many related challenges in the design of MAVs, an important one being the control of flow boundary-layer separation [2] since the flow separation is more likely on the upper surface of the wing at low Reynolds numbers [3]. To solve this problem, researchers have proposed biomimetic methodologies by observing the motion of birds in nature. During the long periods of evolution, birds have developed mature flow control mechanisms that can be applied to a wide variety of engineering designs. For example, during the landing approach, the secondary covert-feathers on the upper surface of bird wings tend to pop up, as shown in Figure 1.

Liebe [4] and Carruthers et al. [5] interpreted this self-adjusting behaviour as a biological high-lift device and assumed that the related delay in flow separation resulted in higher lift at lower flight speeds. Using a wind tunnel, Meyer et al. [6] demonstrated that the lift of an airfoil (HQ17) would increase by about $10 \%$ in nominally stalled conditions by setting self-deploying flexible flaps on its suction side. Similar results were also observed by Schluter and Jorg [1]. Inaoka et al. [7] investigated the feedback flow control of a symmetric airfoil by some mini electromagnetic flap actuators attached on its leading edge, under both steady and unsteady flow conditions in the low Reynolds numbers. They found that the active flap actuators worked well to increase the lift of airfoil and delay the stall angle of attack in the Reynolds number ranging from 30000 to 80000. Bechert et al. [8] conducted a series of wind tunnel experiments and extensively investigated the effects of adaptive flaps mounted on the wing. Their results indicated that for a large aspect ratio wing, adaptive flaps could suppress flow separation, which developed gradually from upstream of the trailing edge, enhancing the maximum lift by up to $20 \%$. John and Schluter [9] did similar work to investigate the three-dimensional effects on the performance of a passive flap on a finite wing, they found the chord-wise location and the size of the flap followed the results of twodimensional airfoil analysis, but the overall performance gain for three-dimensional geometries is lower than observed 
for two-dimensional airfoil analysis. Bramesfeld and Maughmer [10] mounted small, movable flaps on the upper surface of a S824 airfoil and tested its aerodynamic performance in a low-speed wind tunnel at a chord Reynolds number $R e=1.0 \times 10^{6}$. They discovered that the flaps act as pressure dams, which could reduce the adverse effects of the flow separation, allowing lower pressures upstream of their location than would occur otherwise. Compared to the clean airfoil, the maximum lift coefficient could be increased by approximately $20 \%$ with these simple devices.

For the further investigation of this passive separation control mechanism, Johnston et al. [11, 12] compared the effects of free-moving and fixed flaps mounted at different deployment angles over an angle of attack range from $12^{\circ}$ to $20^{\circ}$. They observed a progressive increase in the stall angle of attack with increasing flap angle, with diminishing returns beyond $60^{\circ}$. The oil flow visualization on the airfoil with and without the fixed-angle flaps proved that the effector (flap) caused the separation point to move aft on the airfoil, as compared to the clean airfoil. Bruecker and Weidner [13] investigated various configurations of self-adaptable hairy flaps located on the lower half of a NACA0020 airfoil at low Reynolds number and measured the flow evolution along the airfoil with and without hairy flaps over gradually increasing angles of attack. They interpreted the stall delay as a reduction of the backflow and re-organisation of the shear layer rollup process.

Besides wind tunnel experiments, some researchers have also used numerical methods to investigate the flow separation mechanisms behind this behaviour and have obtained many useful results. Favier et al. [14] numerically simulated flow past a two-dimensional circular cylinder with a cilia-like hairy coating at Reynolds number $R e=200$. They found the overall drag can be reduced by $15 \%$ compared to the clean cylinder. Similar work was done by Venkataraman and Bottaro [15] who simulated a NACA0012 airfoil with hairy coatings attached to its upper surface and found that the coatings could decrease drag oscillations by approximately $11 \%$ and increase lift by approximately $9 \%$ under separated flow conditions. Meyer et al. [6] used the finite volume method to solve the Navier-Stokes equations, simulating twodimensional flow past an HQ17 airfoil with a free-movable flap. They provided a detailed description of the lift enhancement phenomena and the effects of the flap on the flow. In their cases, the free-movable flap is modeled as a solid body and has only one degree of freedom. Rosti et al. did similar work and simulated two-dimensional [16][17] and threedimensional [18] turbulent flow around a NACA0020 airfoil with hairy flaps installed on the suction side and close to the trailing edge during an angle of attack (AoA) ramp-up. In their three-dimensional cases, each flap was still modeled as a lumped one dimensional flexible flap.

So far, the required analysis to find an optimal flap configuration at a given angle of attack and Reynolds number is scarce in the literature. Furthermore, there is a lack of investigations into the flap material properties and detailed explorations on the mechanisms of this kind of separation control. This paper numerically investigates the passive separation control of an airfoil via a flap by using a many-core fluid structure interaction (FSI) solver LUMA [19]. The fluid motion in LUMA is obtained by the lattice Boltzmann method (LBM), and the interaction between fluid and structure is handled by the immersed boundary method (IBM). Recently, the combination of these two methods (IB-LBM) is widely applied in FSI simulations of flexible structures with unsteady flow, such as the large deformation of rectangular plate with zero thickness [20]; the two dimensional flexible artificial heart model [21]; two dimensional [22] and three dimensional [23] flexible capsule; the flexible motion of flaps with finite mass [24], and the flapping dynamics of a flexible flag in a uniform incompressible flow [25]. However, when the Reynolds numbers become high $\left(\operatorname{Re}=10^{3} \sim 10^{5}\right)$, in order to achieve numerically stable simulations, the total computational cost of the standard LBM has to scale with $R e^{D+1}$, where $D$ is the number of dimensions [26], causing a heavy computational burden. For this reason, the Reynolds number in present paper is limited at $R e=1000$. To reduce the computational effort, several strategies have been applied to improve IB-LBM, the relevant work can be found in [26] and [27]. Except for the LBM and IBM, LUMA also introduces the finite element method (FEM) as a powerful structural solver in FSI simulation due to its great compatibility to handle unstructured meshes and good performance in nonlinear dynamic studies [28]. Similar work can also be found in [29] and [30].

In present paper, the NACA0012 airfoil is selected and both rigid and flexible flaps separately attached on the upper surface of the airfoil. The overall aerodynamic performance of the airfoil and flap combination is quantified by the mean lift, mean drag, mean lift-drag ratio, maximum lift fluctuation and maximum drag fluctuation. The effects of flap configurations (length, attachment position, deployment angle and material properties) on the aerodynamic performance are investigated and the separation control mechanism of rigid/flexible flap is explored. 


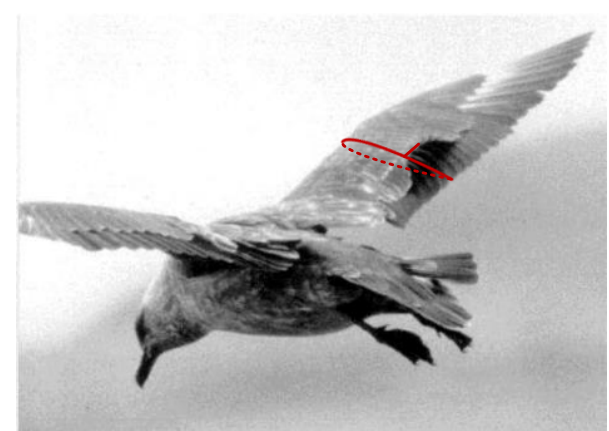

Figure 1: A bird with popped-up secondary covertfeathers (Picture from Rechenberg et al. [45])

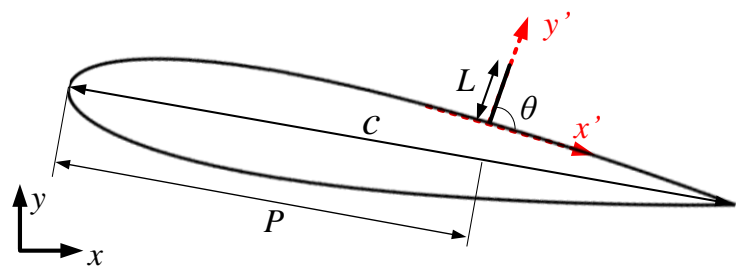

Figure 2: Configuration of a NACA0012 at $\mathrm{AoA}=10^{\circ}$ with a flap attached on its upper surface.

\section{Physical model and numerical method}

The physical model used in this paper is shown in Figure 2. The NACA0012 airfoil has a $10^{\circ}$ AoA and its chord length is $c$. A flap is attached on its upper surface with length $L$, the deployment angle between flap and airfoil upper surface is $\theta$, and the distance from the airfoil leading edge to the fixed end of flap measured along the chord $c$ is $P$.

As introduced in previous section, in the numerical simulation of this model, the fluid motion is obtained by solving the lattice Boltzmann equation, the dynamics of the flexible flap are calculated using the finite element method. The interaction between fluid and structure is handled by the immersed boundary method. In this section, the theoretical formulations of these three methods are described briefly.

\subsection{Lattice Boltzmann method}

As an explicit time-advancement method, the lattice Boltzmann method (LBM) has been proven to be a viable and efficient substitute for Navier-Stokes solver. Based on microscopic models and mesoscopic kinetic equations, the evolutionary process of the LBM is simple and clear, and its computational stencil is local and has natural parallelism, making it highly suited to parallel calculations [31]. The Boltzmann equation for incompressible viscous flow is:

$$
\frac{\partial f}{\partial t}+\mathbf{e} \cdot \nabla_{\mathbf{x}} f+\mathbf{F} \cdot \nabla_{\mathbf{e}} f=\Omega_{f}
$$

where $f$ is the distribution function of the particles located at spatial coordinate $\mathbf{x}$ and time $t$ with velocity e. The force term $\mathbf{F}$ accounts for any external force applied to the fluid, $\Omega_{f}$ represents the collision operator which includes a nonlinear distribution function term $f$. The collision operator can be simplified using the Bhatnagar, Gross, and Krook (BGK) approach [32], which is given by:

$$
\Omega_{f}=\frac{1}{\tau}\left(f^{(e q)}-f\right)
$$

where $\tau$ is the single relaxation time parameter related to the kinematic viscosity of the fluid $v$, and $f^{(e q)}$ is the equilibrium distribution function obtained by a Taylor series expansion of the Maxwell-Boltzmann equilibrium distribution [33]. Equation(1) can be discretised in time and space, and solved on a uniform Cartesian mesh. At each point on the mesh, each particle is assigned one of a finite number of discrete velocity values. In our case we use the D2Q9 model, which refers to a two-dimensional mesh with nine discrete velocities, referred to by subscript $\alpha$. The corresponding equilibrium distribution function $f^{(e q)}$ in $\mathrm{D} 2 \mathrm{Q} 9$ model can be obtained as:

$$
f_{\alpha}^{(e q)}=\rho w_{\alpha}\left[1+\frac{\mathbf{e}_{\alpha} \cdot \mathbf{u}}{c_{s}^{2}}+\frac{\left(\mathbf{e}_{\alpha} \cdot \mathbf{u}\right)^{2}}{2 c_{s}^{4}}-\frac{\mathbf{u}^{2}}{2 c_{s}^{2}}\right]
$$

where $\rho$ is the fluid density, $c_{s}=c / \sqrt{3}$ is the speed of sound, $c$ is the lattice speed defined by $c=\delta x / \delta t=1, \delta x$ and $\delta t$ represent the lattice size and time step, respectively. $\mathbf{e}_{\alpha}$ and $w_{\alpha}$ are nine discrete velocities and corresponding weight coefficients, which take standard values [31]. It should be noted that the Mach number of the macroscopic velocity $\mathbf{u}$ in lattice units must be far less than 1, which is equivalent to the CFL number in classical Navier-Stokes solvers. In this model, the relationship between single relaxation time $\tau$ and the kinematic viscosity of fluid $v$ is:

$$
v=\left(\tau-\frac{1}{2}\right) c_{s}^{2} \delta t
$$

For the discretization of the external forcing term $\mathbf{F}$, we use the representation proposed by Guo et al. [34] which accounts for the contribution of the additional force to the momentum and momentum flux. The expression can be written as: 


$$
F_{\alpha}=\left(1-\frac{1}{2 \tau}\right) w_{\alpha}\left[\frac{\mathbf{e}_{\alpha}-\mathbf{u}}{c_{s}^{2}}+\frac{\mathbf{e}_{\alpha} \cdot \mathbf{u}}{c_{s}^{4}} \mathbf{e}_{\alpha}\right] \cdot \mathbf{f}
$$

where $\mathbf{f}$ is the force density acting on the fluid. Then the macroscopic fluid variables can be calculated by:

$$
\begin{gathered}
\rho=\sum_{\alpha} f_{\alpha} \\
\rho \mathbf{u}=\sum_{\alpha} \mathbf{e}_{\alpha} f_{\alpha}+\frac{1}{2} \mathbf{f} \delta t
\end{gathered}
$$

\subsection{Finite element method}

The FEM is introduced as the structural solver to obtain the dynamic response of flexible flaps. In addition, the geometrical non-linearity is also taken into consideration. In the non-linear dynamic FEM [35], the kinetic equation of flexible flap can be written as:

$$
\mathbf{M} \ddot{\mathbf{X}}(t)+\mathbf{C X}(t)+\mathbf{F}_{i n t}(\mathbf{X})-\mathbf{F}_{\text {ext }}(t)=0
$$

where $\mathbf{X}, \dot{\mathbf{X}}$ and $\ddot{\mathbf{X}}$ represent the displacement, velocity and acceleration of boundary points (or Lagrangian points in IBM, see in Section 2.3), respectively. $\mathbf{M}$ and $\mathbf{C}$ are the mass and damping matrices of the flap, in present work we do not consider the flap damping, so matrix $\mathbf{C}$ equals zero. $\mathbf{F}_{\text {int }}$ represents the internal force of the flap, it is a non-linear function of the displacement $\mathbf{X}$. $\mathbf{F}_{\text {ext }}(t)$ represents the external forces such as the fluid force and gravity acting on the flap.

Equation(8) can be solved by the Newmark- $\beta$ method [36] which is also an implicit method. In this method, the acceleration and velocity of flap can be written as:

$$
\begin{aligned}
& \ddot{\mathbf{X}}_{t+\Delta t}=c_{0}\left(\mathbf{X}_{t+\Delta t}-\mathbf{X}_{t}\right)-c_{2} \dot{\mathbf{X}}_{t}-c_{3} \ddot{\mathbf{X}}_{t} \\
& \dot{\mathbf{X}}_{t+\Delta t}=c_{1}\left(\mathbf{X}_{t+\Delta t}-\mathbf{X}_{t}\right)-c_{4} \dot{\mathbf{X}}_{t}-c_{5} \ddot{\mathbf{X}}_{t}
\end{aligned}
$$

where $c_{0} \sim c_{5}$ are six variables which are related to the accuracy and stability requirements of the method, and their specific expressions can be found in [36].

In order to obtain the displacement of flap at time $t+\Delta t$, we use the Newton-Raphson method to process the non-linear item $\mathbf{F}_{\text {int }}(\mathbf{X})$ in Equation(8). In this method, we assume that the non-linear internal force of flap $\mathbf{F}_{\text {int }}$ at time $t+\Delta t$ can be expressed as:

$$
\mathbf{F}_{i n t, t+\Delta t}=\mathbf{F}_{i n t, t}+\frac{\partial \mathbf{F}_{i t t}}{\partial \mathbf{X}} \Delta \mathbf{X}=\mathbf{F}_{i n t, t}+K_{r, t} \Delta \mathbf{X}
$$

where $K_{T}$ is the global tangent stiffness matrix of the flap, it can be assembled by the element stiffness matrix:

$$
K_{T}=\sum_{e} K_{T}^{e}=\sum_{e}\left[K_{E}^{e}+K_{G}^{e}\right]=\sum_{e} T^{e T}\left[\bar{K}_{E}^{e}+\bar{K}_{G}^{e}\right] T^{e}
$$

where $T^{e}$ is the transformation matrix from local coordinate system to global coordinate system. $\bar{K}_{E}^{e}$ is the local element elastic stiffness matrix which corresponds to the elastic deformation of structure. $\bar{K}_{G}^{e}$ is the local element geometric stiffness matrix which is also a function of the displacement $\mathbf{X}$. The expressions of $\bar{K}_{E}^{e}$ and $\bar{K}_{G}^{e}$ are determined by the element type. For the flexible flap, the corresponding element chosen here is beam element, the expressions of $\bar{K}_{E}^{e}$ and $\bar{K}_{G}^{e}$ can be written as [35]:

$$
\begin{gathered}
\bar{K}_{E}^{e}=\frac{E A}{L}\left[\begin{array}{cccccc}
1 & 0 & 0 & -1 & 0 & 0 \\
0 & 0 & 0 & 0 & 0 & 0 \\
0 & 0 & 0 & 0 & 0 & 0 \\
-1 & 0 & 0 & 1 & 0 & 0 \\
0 & 0 & 0 & 0 & 0 & 0 \\
0 & 0 & 0 & 0 & 0 & 0
\end{array}\right]+\frac{E I}{L^{3}}\left[\begin{array}{cccccc}
0 & 0 & 0 & 0 & 0 & 0 \\
0 & 12 & 6 L & 0 & -12 & 6 L \\
0 & 6 L & 4 L^{2} & 0 & -6 L & 2 L^{2} \\
0 & 0 & 0 & 0 & 0 & 0 \\
0 & -12 & -6 L & 0 & 12 & -6 L \\
0 & 6 L & 2 L^{2} & 0 & -6 L & 4 L^{2}
\end{array}\right] \\
\bar{K}_{G}^{e}=\frac{\bar{F}_{x}}{L}\left[\begin{array}{cccccc}
0 & 0 & 0 & 0 & 0 & 0 \\
0 & 1 & 0 & 0 & -1 & 0 \\
0 & 0 & 0 & 0 & 0 & 0 \\
0 & 0 & 0 & 0 & 0 & 0 \\
0 & -1 & 0 & 0 & 1 & 0 \\
0 & 0 & 0 & 0 & 0 & 0
\end{array}\right]-\frac{\bar{F}_{y}}{L}\left[\begin{array}{cccccc}
0 & 1 & 0 & 0 & -1 & 0 \\
1 & 0 & 0 & -1 & 0 & 0 \\
0 & 0 & 0 & 0 & 0 & 0 \\
0 & -1 & 0 & 0 & 1 & 0 \\
-1 & 0 & 0 & 1 & 0 & 0 \\
0 & 0 & 0 & 0 & 0 & 0
\end{array}\right]
\end{gathered}
$$


where $L, A, E$ and $I$ are the length, cross section area, Young's modulus and cross sectional moment of inertia of the beam elements (flexible flap). $\bar{F}_{x}$ and $\bar{F}_{y}$ are the normal stress and shear stress of beam elements in local coordinate system. Based on the above discussion, the final expression of Equation(8) can be written as:

$$
\begin{aligned}
{\left[K_{T, t+\Delta t}^{i}+c_{0} \mathbf{M}+c_{1} \mathbf{C}\right] \Delta \mathbf{X}^{i+1}=} & F_{e x t, t+\Delta t}-F_{\mathrm{int}, t+\Delta t}^{i} \\
& -\mathbf{M}\left[c_{0}\left(\mathbf{X}_{t+\Delta t}^{i}-\mathbf{X}_{t}\right)-c_{2} \dot{\mathbf{X}}_{t}-c_{3} \ddot{\mathbf{X}}_{t}\right] \\
& -\mathbf{C}\left[c_{1}\left(\mathbf{X}_{t+\Delta t}^{i}-\mathbf{X}_{t}\right)-c_{4} \dot{\mathbf{X}}_{t}-c_{5} \ddot{\mathbf{X}}_{t}\right]
\end{aligned}
$$

Because $K_{T}, \mathbf{M}$ and $\mathbf{C}$ are symmetric matrices, the above iteration can be solved by the triangular decomposition method. When the above iteration converges, the displacement of flap $\mathbf{X}_{t+\Delta t}$ can be obtained. Substituting $\mathbf{X}_{t+\Delta t}$ into Equation(9), the acceleration $\ddot{\mathbf{X}}_{t+\Delta t}$ and velocity $\dot{\mathbf{X}}_{t+\Delta t}$ of flap can also be obtained.

\subsection{Immersed boundary method}

The basic idea of the immersed boundary method [37] is that the flow field is influenced by the force distribution generated by the boundary of the structure immersed in the fluid. The immersed boundary method uses independent grids to discretize the fluid and structure separately. As shown in Figure 3, the fluid is discretized by a set of Eulerian points, which are in fact the fixed regular Cartesian lattice points, while the boundary of the structure immersed in the fluid is discretized by a set of markers which are called Lagrangian points. The blue domain depicted by red dash lines is the fluid region which is influenced by one Lagrangian point. When the structure boundary has a small distortion, there will be a force acting on the Lagrangian point that tries to restore the boundary back to its original position. This force will be distributed on the Eulerian grid and then the flow field is updated.

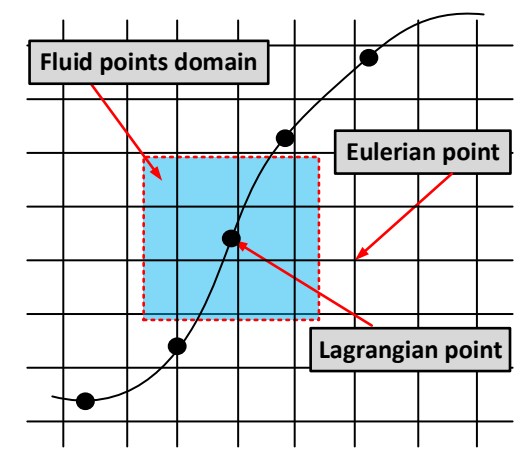

Figure 3: Immersed boundary illustration.

Since the fluid grid and structure grid are separate from each other, communication between them is crucial. Moreover, since they are non-conforming, this data transfer requires specialised interpolation/spreading operators. Specifically, the momentum field must be interpolated from the fluid grid (Eulerian) to the boundary grid (Lagrangian), and the resulting forces must be spread back from the boundary grid to the fluid grid. The operators defining these communication steps are given by [38]:

$$
\begin{gathered}
\Phi(\mathbf{X})=\Pi[\phi(\mathbf{x})]=\sum_{\Omega} \phi(\mathbf{x}) \tilde{\delta}(\mathbf{x}-\mathbf{X}) \Delta x \Delta y \Delta z \\
\phi(\mathbf{x})=\Psi[\Phi(\mathbf{X})]=\sum_{\Gamma} \Phi(\mathbf{X}) \tilde{\delta}(\mathbf{x}-\mathbf{X}) \grave{\mathrm{o}} \Delta q \Delta r \Delta s
\end{gathered}
$$

where $\mathrm{x}=(x, y, z)$ is the position of fluid point in Eulerian frame, $\mathrm{X}=(q, r, s)$ is the positon of structure boundary point in Lagrangian frame, $\Omega$ is the computational domain and $\Gamma$ is the boundary of the structure. $\phi$ is a quantity defined in the Eulerian frame, $\Phi$ is the same quantity defined in the Lagrangian frame and $\epsilon$ is a scaling factor which ensures reciprocity between the interpolation and spreading steps. Here, lower-case notation denotes values in the Eulerian frame, and uppercase notation denotes values in the Lagrangian frame. The present work adopts the three-point version of the discrete Dirac delta function $\tilde{\delta}$, proposed by Roma et al. [39].

Using the algorithm given by Li et al. [40] and $\mathrm{Wu} \& \mathrm{Shu}$ [41], the fluid velocity is decomposed into a predicted and a force-corrected term. Based on Equation(7), after one pass of the LBM equations, the macroscopic fluid velocity can be written as:

$$
\rho \mathbf{u}=\rho \mathbf{u}^{*}+\frac{1}{2} \mathbf{f} \delta t
$$


where $\mathbf{u}^{*}$ is the predicted velocity. The density and predicted velocity field are the only known quantities at this stage. Since the fluid velocity at the boundary must equal the velocity of the boundary $\mathbf{U}=\Pi[\mathbf{u}]$, then converting Equation(17) into the Lagrangian frame gives:

$$
\Pi[\rho(\mathbf{x}, t)] \mathbf{U}(\mathbf{X}, t)=\Pi\left[\rho \mathbf{u}^{*}(\mathbf{x}, t)\right]+\frac{\Delta t}{2} \mathbf{F}(\mathbf{X}, t)
$$

Since the velocity of the boundary $\mathbf{U}$ is known, Equation(18) can be rearranged and solved for the corrective force density F. The force is then transferred back to the Eulerian frame via the spreading operator $\Psi$. Finally, the velocity field is updated by adding the corrective force to the predicted velocity (Equation(17)).

\section{Results and discussion}

In this section, the flow around a NACA0012 airfoil with and without a rigid/flexible flap is investigated, and the effects of the flap configurations (length, attachment position, deployment angle and material properties) on the airfoil aerodynamic performance are analyzed. The airfoil aerodynamic performance is quantified by the overall mean lift and drag coefficients, mean lift-drag ratio, maximum lift and drag fluctuation.

\subsection{Flow around a NACA0012 airfoil}

Before exploring the effects of the flap on the aerodynamic performance of the NACA0012 airfoil, we first study the unsteady flow around a clean (or single) NACA0012 airfoil at $R e=1000$ and $\mathrm{AoA}=10^{\circ}$, which has been investigated extensively in the literature. As shown in Figure 4, in the present simulation the computational fluid domain is $\mathrm{X} \times \mathrm{Y}=50 c \times 40 c$, the leading edge of airfoil is put at the location $(20 c, 20 c)$ and the airfoil surface is represented by 500 Lagrangian points. There are four levels of grid refinement (Grid 1 to Grid 4) in the whole fluid domain and their relative positions are shown in this figure. Most region of fluid domain is covered by a coarse mesh with a resolution of $\Delta \mathrm{x}=\Delta \mathrm{y}=0.04 c$, while the region around the airfoil $(4 c \times 2 c)$ is discretized by a very fine uniform mesh with a resolution of $\Delta \mathrm{x}=\Delta \mathrm{y}=0.0025 \mathrm{c}$. In the lattice Boltzmann method, the lattice freestream velocity of flow is $U_{l b}=0.05$ and the fluid density is $\rho=1.0$. The Strouhal number $S t$ here is defined as $S t=f_{D} c / U_{\infty}$, where $f_{D}$ is the vortex shedding frequency, $U_{\infty}$ is the freestream velocity.

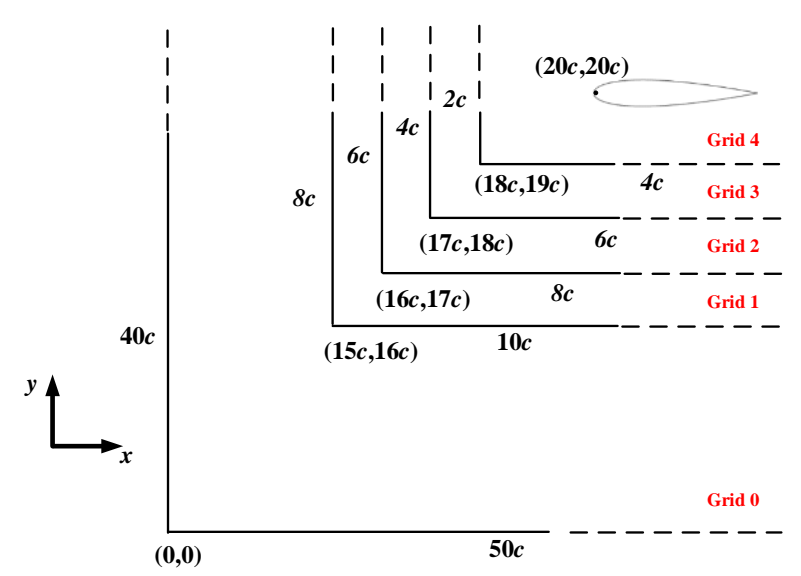

Figure 4: The sketch of grid refinement in computational fluid domain.

Figure 5a shows how the lift and drag coefficients of NACA0012 airfoil change with time in the present case. It can be seen from this figure that at $R e=1000$ and $\mathrm{AoA}=10^{\circ}$, the lift and drag coefficient variations in time are in phase with the maximum drag (occurring at maximum lift) and minimum drag (occurring at minimum lift). In Table 1 the obtained aerodynamic performance results are compared with results reported by Falagkaris et al. [42] and Mittal \& Tezduyar [43], it can be indicated from this table that our results agree well with them. The obtained time-averaged lift coefficient over the last six periods is compared with the results in [42] and [43] in Figure 5b, it can be seen that the present results agree well with these two references. Figure 5c shows the snapshot of fluid field vorticity contour, it can be observed in this figure that there are positive and negative vortices shed alternately from the tail of airfoil, which diffuse as they convect downstream forming a Von Karman vortex street. This behaviour causes the lift and drag of airfoil to periodically oscillate with time. 
Table 1: The aerodynamic performance results of clean NACA0012 airfoil at $R e=1000$ and AoA $=10^{\circ}$

\begin{tabular}{|c|c|c|c|}
\hline & Present & Falagkaris et al.[42] & Mittal \& Tezduyar [43] \\
\hline Mean lift coefficient $\bar{C}_{L}$ & 0.4203 & $\mathbf{0 . 4 1 7 8}$ & $\mathbf{0 . 4 2 2 1}$ \\
\hline Mean drag coefficient $\bar{C}_{D}$ & 0.1674 & 0.1667 & 0.1650 \\
\hline Mean lift-drag ratio $\bar{C}_{L} / \bar{C}_{D}$ & 2.5108 & 2.5063 & 2.5581 \\
\hline Maximum lift coefficient fluctuation $C_{L}^{\prime}$ & 0.0510 & 0.0460 & 0.0426 \\
\hline Maximum drag coefficient fluctuation $C_{D}^{\prime}$ & 0.0053 & 0.0049 & 0.0048 \\
\hline
\end{tabular}

(a)

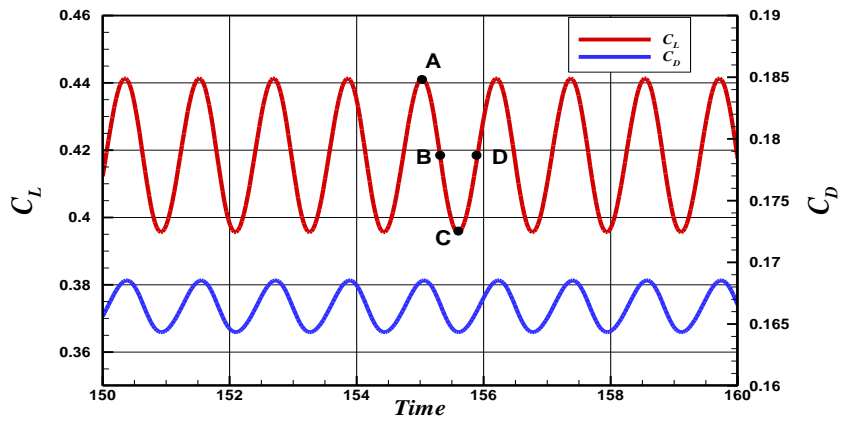

(b)

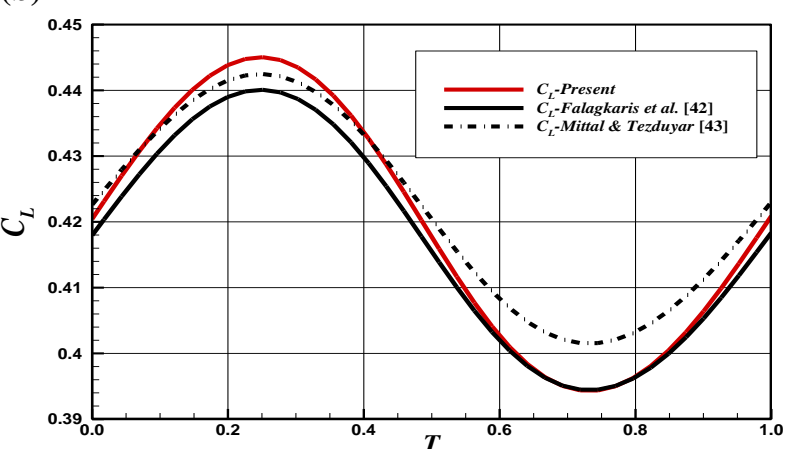

(c)

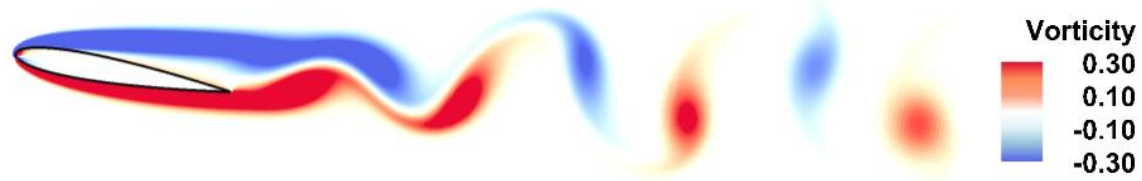

Figure 5. (a): Time evolution of lift and drag coefficients for the flow around NACA0012 airfoil at $R e=1000$ and AoA=10 . (b): Average lift coefficient with $T$. (c): Snapshot of fluid field vorticity contour.

\subsection{Flow around a NACA0012 airfoil with a rigid flap}

After evaluating the aerodynamic performance of the clean NACA0012 airfoil, in this section we attach one rigid flap on its upper surface and investigate the effects of flap configurations on its aerodynamic performance. To describe the length and attachment position of flap, we define two dimensionless parameters: the length ratio $L^{*}=L / c$ and the position ratio $P^{*}=P / c$, and their values are listed in Table 2. According to the experimental study of John and Schluter [9], the flap close to the airfoil leading edge has only minor effects on its aerodynamic performance, so we choose five different positions $\left(P^{*}=0.5,0.6,0.7,0.8\right.$ and 0.9$)$ on the rear part of airfoil upper surface. The length ratio $L^{*}$ of flap is chosen from 0.01 to 0.16 . The deployment angle of flap $\theta$ is fixed at $90^{\circ}$.

Table 2: The values of length ratio $L^{*}$ and position ratio $P^{*}$

\begin{tabular}{cccccccccccc}
$L^{*}$ & 0.01 & 0.02 & 0.03 & 0.04 & 0.05 & 0.06 & 0.08 & 0.10 & 0.12 & 0.14 & 0.16 \\
\hline$P^{*}$ & 0.5 & 0.6 & 0.7 & 0.8 & 0.9 & & & & & & \\
\hline
\end{tabular}

Figure $6 \mathrm{a}$ and $6 \mathrm{~b}$ show the overall mean lift coefficient $\bar{C}_{L}$ and mean drag coefficient $\bar{C}_{D}$ of the NACA0012 airfoil with the rigid flap for each case. For comparison, these results are normalized by the corresponding results of clean airfoil obtained in previous section. From these two figures, it can be seen that for a rigid flap of a fixed length, when it is closer to the trailing edge, the mean lift coefficient increases and the mean drag coefficient decreases. When the flap length is very small $\left(L^{*}=0.01,0.02,0.03\right.$ and 0.04$)$, wherever the flap is put, the airfoil with flap always have a higher mean lift coefficient and smaller mean drag coefficient than the clean airfoil, even though these differences are very small. Conversely, when the flap is long $\left(L^{*}=0.14\right.$ and 0.16$)$, wherever the flap is put, the mean lift coefficient is smaller and mean drag coefficient larger than the clean airfoil. For flap lengths between these two situations $\left(L^{*}=0.06,0.080 .1\right.$ and $0.12)$, when the flap is put close to the leading edge of airfoil $\left(P^{*}=0.5\right)$, it has negative effect on the aerodynamic 
performance of airfoil - smaller mean lift coefficient and larger mean drag coefficient than the clean airfoil. As the rigid flap is moved closer to the trailing edge of airfoil $\left(P^{*}=0.9\right)$, this negative effect will lessen, eventually becoming a positive effect on the aerodynamic performance.

For a rigid flap with a fixed position, when its length increases, the mean lift coefficient increases first and then decreases. Analogously, the mean drag coefficient decreases first and then increases. With the position of flap closer to the trailing edge, the optimal length of flap increases $\left(L^{*}{ }_{\text {opt }}=0.03\right.$ for $P^{*}=0.5$ and $L^{*}{ }_{\text {opt }}=0.1$ for $\left.P^{*}=0.9\right)$. This phenomenon is similar to the observation of John and Schluter [9] in their experimental studies, even though their research object is a three-dimensional SD8020 finite wing.

Figure $6 \mathrm{c}, 6 \mathrm{~d}$ and $6 \mathrm{e}$ show the mean lift-drag ratio $\bar{C}_{L} / \bar{C}_{D}$, maximum lift fluctuation $C_{L}^{\prime}$ and maximum drag fluctuation $C_{D}^{\prime}$ of NACA0012 airfoil together with rigid flap in these test cases, respectively, and these results are also normalized by the clean airfoil case for comparison. From these three figures, it can be found that the variation of $\bar{C}_{L} / \bar{C}_{D}$ is similar to the mean lift coefficient $\bar{C}_{L}$ (as shown in Figure 6a) while the variation of $C_{L}^{\prime}$ and $C_{D}^{\prime}$ are very similar to the mean drag coefficient $\bar{C}_{D}$ (as shown in Figure $6 \mathrm{~b}$ ), so we can draw similar conclusions here. Some combinations of length ratio $L^{*}$ and position ratio $P^{*}$ introduce smaller mean lift-drag ratio and stronger variations in lift and drag coefficient and compared with the clean airfoil, others introduce higher mean lift-drag ratio and smaller variations. These combinations correspond exactly to those which produce the improved and degraded aerodynamic performance illustrated by Figure 6a and $5 \mathrm{~b}$.

Thus, it can be concluded that when $L^{*}=0.1$ and $P^{*}=0.9$, the airfoil with a rigid flap achieves its optimal aerodynamic performance: highest mean lift coefficient, smallest mean drag coefficient, highest mean lift-drag ratio, smallest lift fluctuation and smallest drag fluctuation. Compared to the clean airfoil, the mean lift coefficient of present case is increased by $9.48 \%$, the mean drag coefficient is decreased by $2.55 \%$, the mean lift-drag ratio is increased by $12.34 \%$, the maximum lift fluctuation is decreased by $40.54 \%$ and the maximum drag fluctuation is decreased by $56.65 \%$. Focusing on this optimal case, in the next step we investigate the effects of deployment angle of flap $\theta$ on the aerodynamic performance of airfoil.

Figure 7a shows the overall mean lift coefficient $\bar{C}_{L}$, mean drag coefficient $\bar{C}_{D}$ and mean lift-drag ratio $\bar{C}_{L} / \bar{C}_{D}$ of the NACA0012 airfoil together with a rigid flap ( $L^{*}=0.1, P^{*}=0.9$ ) whose deployment angle $\theta$ changes from $10^{\circ}$ to $90^{\circ}$. These results are normalized by the clean airfoil case for comparison. From this figure, it can be seen that when the deployment angle $\theta$ is very small $\left(10^{\circ} \leqslant \theta \leqslant 20^{\circ}\right)$, the airfoil with flap has smaller mean lift coefficient $\bar{C}_{L}$, mean $\operatorname{drag}$ coefficient $\bar{C}_{D}$ and mean lift-drag ratio $\bar{C}_{L} / \bar{C}_{D}$ than the clean airfoil. As the deployment angle $\theta$ increases, the mean lift coefficient $\bar{C}_{L}$ and mean lift-drag ratio $\bar{C}_{L} / \bar{C}_{D}$ increase while the mean drag coefficient $\bar{C}_{D}$ decreases. In the range of $30^{\circ} \leqslant \theta \leqslant 60^{\circ}$, all $\bar{C}_{L}, \bar{C}_{D}$ and $\bar{C}_{L} / \bar{C}_{D}$ change almost linearly with $\theta$. And at $\theta=90^{\circ}$, the airfoil with flap achieves maximum mean lift coefficient, maximum mean lift-drag ratio and minimum mean drag coefficient. Figure $7 \mathrm{~b}$ shows the normalized maximum lift fluctuation $C_{L}^{\prime}$ and maximum drag fluctuation $C_{D}^{\prime}$ of airfoil together with rigid flap. This figure shows that when the deployment angle $\theta$ is small $\left(10^{\circ} \leqslant \theta \leqslant 30^{\circ}\right)$, the airfoil with flap has a higher maximum lift fluctuation than the clean airfoil. When the deployment angle $\theta$ increases, the maximum lift fluctuation $C_{L}^{\prime}$ increases first and then decreases, at $\theta=20^{\circ}$ with a maximum value of $C_{L}^{\prime}=1.0781$. Analogously, the maximum drag fluctuation $C_{D}^{\prime}$ decreases first and then increases, at $\theta=50^{\circ}$ with a minimum value of $C_{D}^{\prime}=0.3844$.

Based on the discussion above, it can be concluded that at $\theta=90^{\circ}$, the airfoil with a rigid flap achieves its optimal aerodynamic performance: highest mean lift coefficient, smallest mean drag coefficient and smallest maximum lift fluctuation.

(a)

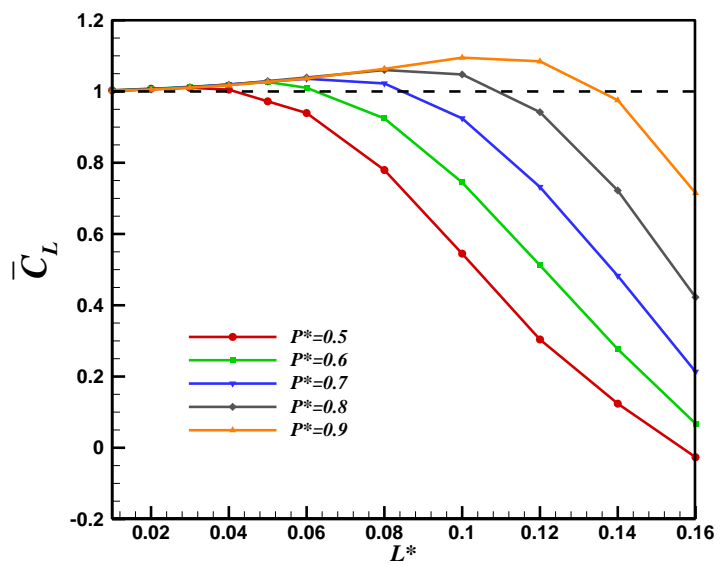

(b)

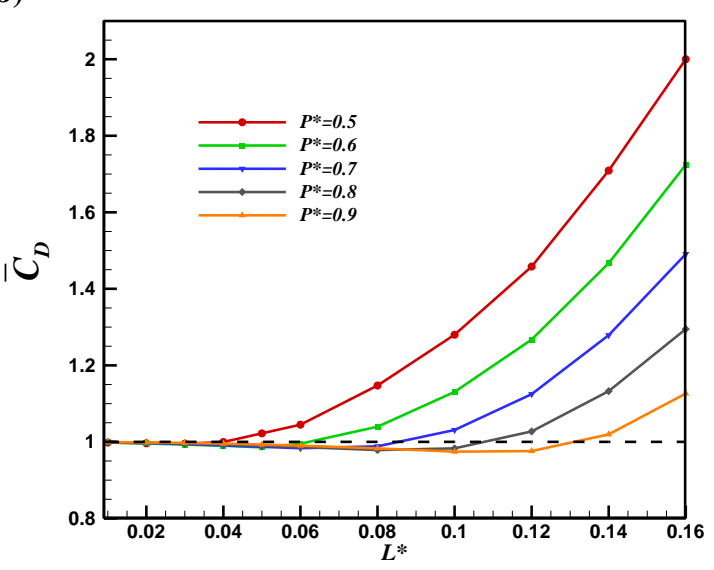


(c)

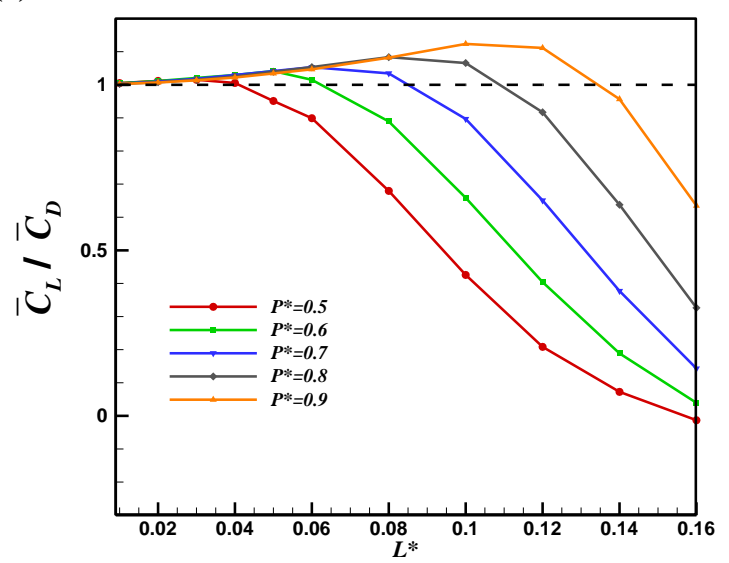

(d)

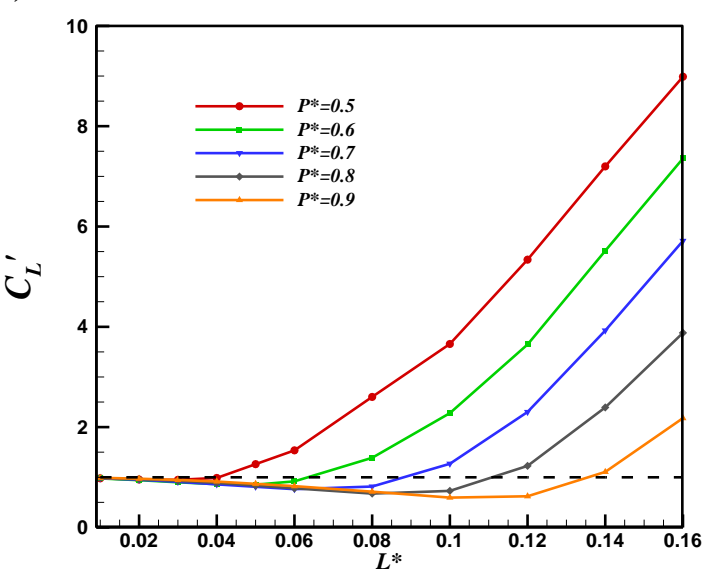

(e)

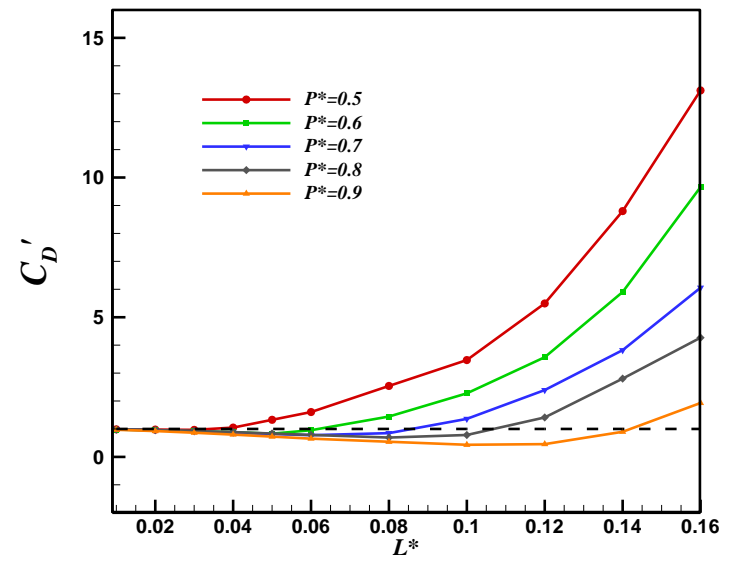

Figure 6. Results for airfoil with rigid flap (a): The mean lift, (b) mean drag and (c) mean lift-drag ratio. (d): maximum lift and (e) maximum drag fluctuation.

(a)

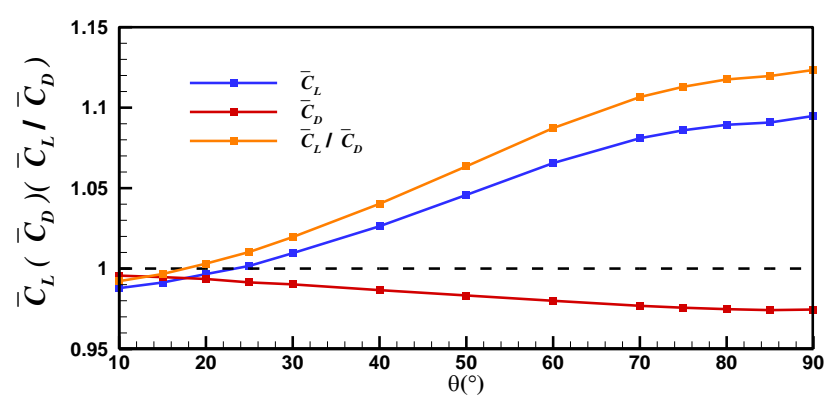

(b)

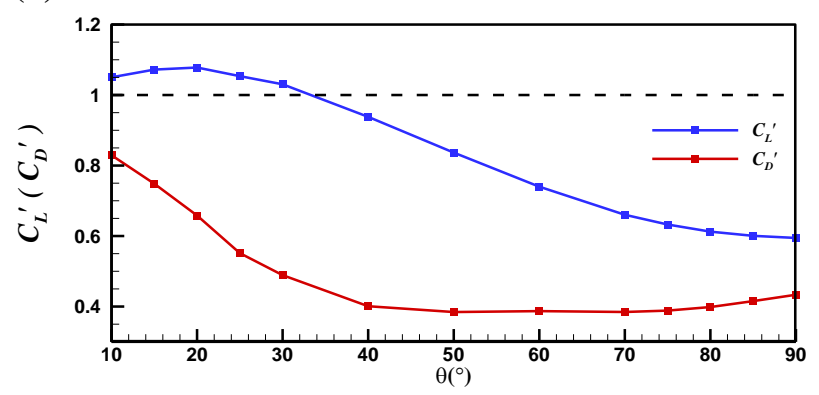

Figure 7. Results for airfoil with rigid flap at different deployment angles for the max $L^{*}=0.1, P^{*}=0.9$ (a): mean lift, drag and liftdrag ratio (b): maximum lift and drag fluctuations.

\subsection{Flow around a NACA0012 airfoil with a flexible flap}

Until now we have explored the effects of a rigid flap on the aerodynamic performance of NACA0012 airfoil, and obtained a partially-optimized flap configuration $\left(L^{*}=1.0, P^{*}=0.9\right.$ and $\left.\theta=90^{\circ}\right)$. In this section we use this configuration as a benchmark and replace the rigid flap with a flexible flap. The root of flexible flap is clamped on airfoil surface in order to keep the deployment angle $\theta$ constant. To describe the material properties of flexible flap, we define two dimensionless parameters: the bending coefficient $K^{*}=E I /\left(\rho_{f} U_{\infty}^{2} L^{3}\right)$ and the mass ratio $M^{*}=\rho_{s} h / \rho_{f} L$, where $h$ is the flap thickness, $\rho_{s}$ is the flap density and $\rho_{f}$ is the fluid density chosen as $\rho_{f}=1.225 \mathrm{~kg} / \mathrm{m}^{3}$.

To describe the flap deformation clearly, a new coordinate system $x^{\prime} y^{\prime}$ is established in which two axes are normal and tangential to the flap equilibrium position, respectively, as shown in Figure 2. Figure 8a and 8b show the mean $x$ 'displacement $\bar{D}_{x^{\prime}}$ and $x^{\prime}$-displacement vibration amplitude $\Delta D_{x^{\prime}}$ of flap tip normalised by the flap length $L$. The flap bending coefficient $K^{*}$ varies from 0.01 to 2 and the flap mass ratio $M^{*}$ varies from 5 to 50 . It can be seen from these two Figures that as the flap tends to becoming rigid ( $K^{*}$ increases), the mean $x$-displacement $\bar{D}_{x^{\prime}}$ of its tip increases while the 
$x^{\prime}$-displacement vibration amplitude $\Delta D_{x^{\prime}}$ decreases. When $K^{*} \geqslant 1.5$, both $\bar{D}_{x^{\prime}}$ and $\Delta D_{x^{\prime}}$ tend to zero. When the flap becomes heavier ( $M^{*}$ increases), its tip $x^{\prime}$-displacement has a smaller mean value but bigger vibration amplitude. As the mean $x^{\prime}$-displacement of flap tip $\bar{D}_{x^{\prime}}$ is always negative, the mean value of $\bar{D}_{x^{\prime}}$ may therefore be bigger than $\Delta D_{x^{\prime}}$, this indicates the asymmetry of the oscillations with the flap oscillating always on one side of its starting position. When the value of $\bar{D}_{x^{\prime}}$ is smaller than $\Delta D_{x^{\prime}}$, this indicates that the flapping motion is more symmetrical. These two different flapping patterns will be discussed in next section.
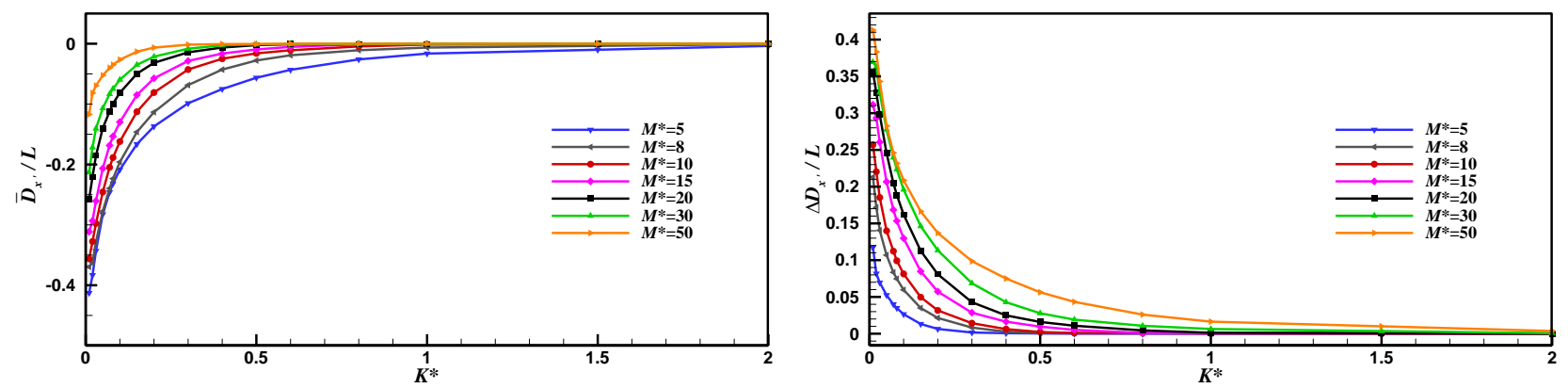

Figure 8. (a): The mean $x$ '-displacement and (b): The $x$ '-displacement vibration amplitude of flap tip for different cases.

Figure 9a, 9b, 9c, 9d and 9e show the overall mean lift coefficient $\bar{C}_{L}$, mean drag coefficient $\bar{C}_{D}$, mean lift-drag ratio $\bar{C}_{L} / \bar{C}_{D}$, maximum lift fluctuation $C_{L}^{\prime}$ and maximum drag fluctuation $C_{D}^{\prime}$ of the NACA0012 airfoil together with a flexible flap in different test cases. For comparison, the results are normalized by the corresponding results of airfoil with a rigid flap case obtained in previous section. For $0.5 \leqslant \mathrm{~K}^{*} \leqslant 2$, because the differences in the results of these cases are very small, we do not include them in the figures. Figure 9 shows that the mean lift coefficient $\bar{C}_{L}$, the mean drag coefficient $\bar{C}_{D}$, the mean lift-drag ratio $\bar{C}_{L} / \bar{C}_{D}$, the maximum lift fluctuation $C_{L}^{\prime}$ and the maximum $\operatorname{drag}$ fluctuation $C_{D}^{\prime}$ have variations similar to the rigid cases shown in Figure 6. So we discuss the results shown in Figure 9a in detail as with earlier results, analogous conclusions can be drawn from Figure 9b, 9c, 9d and 9e.

From Figure 9a, it can be seen that when the flap stiffness $K^{*}$ is very small, the airfoil with a lighter flap ( $M^{*}$ is small) has a higher mean lift coefficient. For the flap with $M^{*}=5$, the mean lift coefficient is higher than that in rigid flap case, while for the flap with $M^{*}=50$, the mean lift coefficient is much smaller than that in rigid flap case, even smaller than that in the clean airfoil case. For a fixed mass ratio $M^{*}$, as $K^{*}$ increases, the mean lift coefficient of the airfoil with the flexible flap increases first and then reaches a peak (except for $M^{*}=5$ ). For smaller mass ratios, this peak is reached more quickly. For $M^{*}=15$, the mean lift coefficient has the highest peak value of $\bar{C}_{L}=1.0368$. As $K^{*}$ continues to increase, the mean lift coefficient decreases from the peak tending to the results of rigid flap case. The heavier flaps ( $M *$ is bigger) generally have higher values of lift during this descent.

From the above discussion, it can be concluded that when $K^{*}=0.08$ and $M^{*}=15$, the airfoil with a flexible flap achieves its optimal aerodynamic performance: highest mean lift coefficient, smallest mean drag coefficient, highest mean liftdrag ratio, smallest lift fluctuation and smallest drag fluctuation. Compared to the rigid flap case, the mean lift coefficient of the present case is improved by $3.68 \%$, the mean drag coefficient is decreased by $1.15 \%$, the mean lift-drag ratio is improved by $4.89 \%$, while the maximum lift and drag fluctuation are both decreased by $0.60 \%$. Compared to the clean airfoil case, the mean lift coefficient of present case is improved by $13.51 \%$, the mean drag coefficient is decreased by $3.67 \%$, the mean lift-drag ratio is improved by $17.84 \%$, the maximum lift fluctuation is decreased by $40.90 \%$ and the maximum drag fluctuation is decreased by $56.90 \%$.

(a)

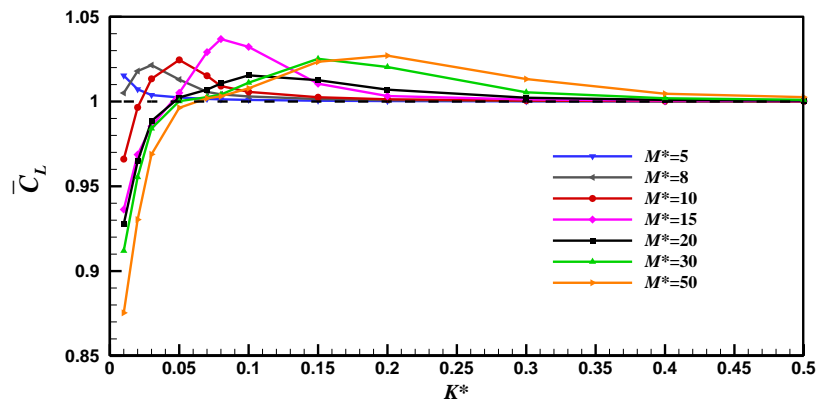

(b)

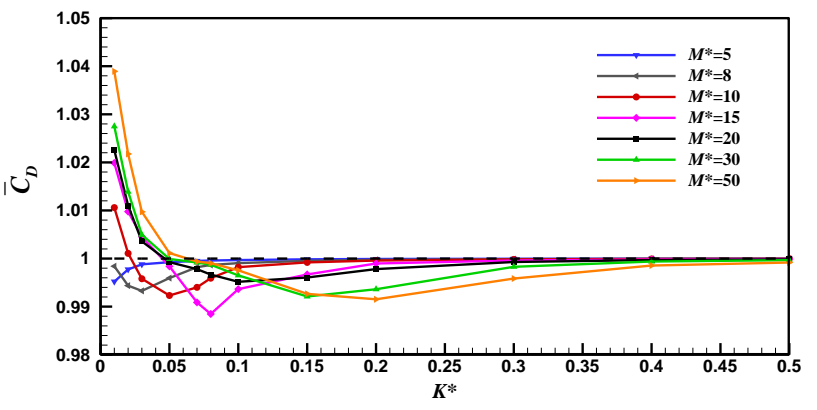


(c)

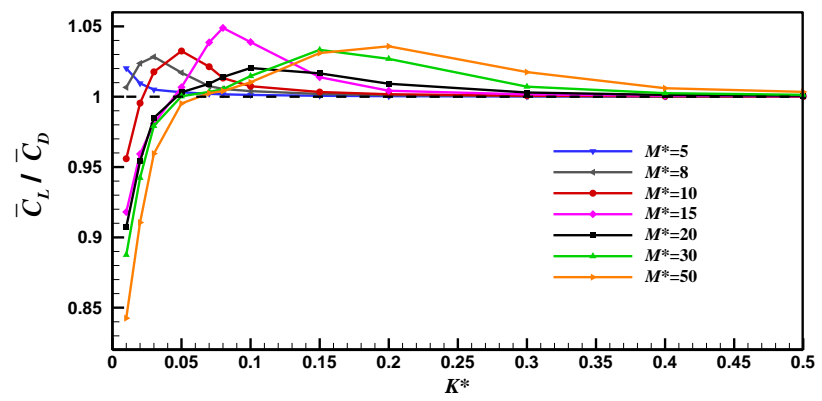

(d)

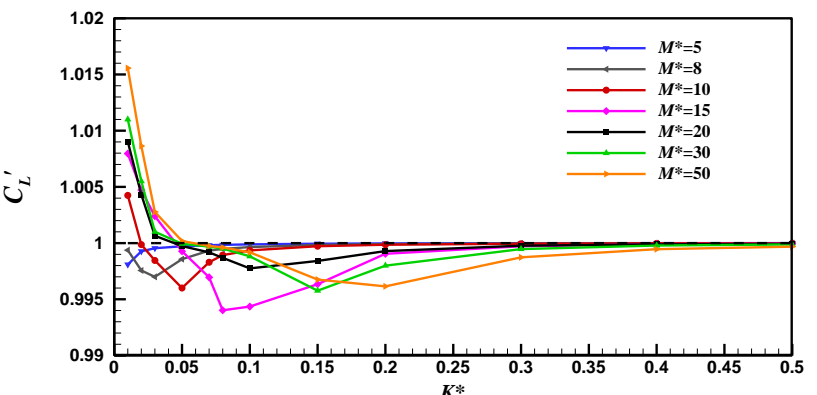

(e)

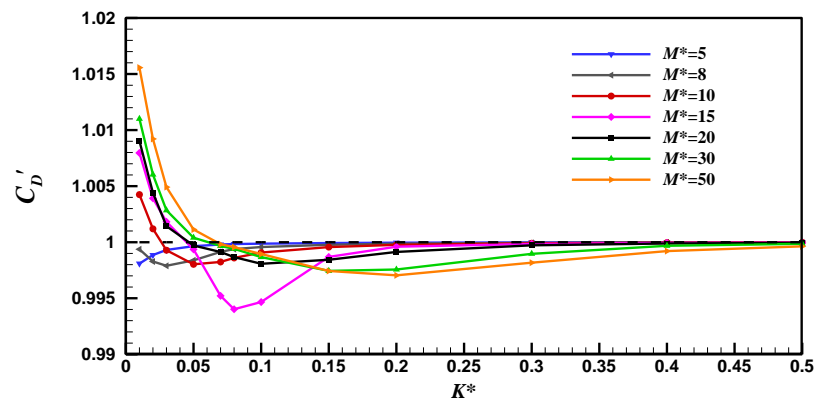

Figure 9. Results for airfoil with flexible flap (a): mean lift, (b): mean drag (c): mean lift-drag ratio (d): maximum lift fluctuation (e): maximum drag fluctuation.

\section{Mechanism of separation control via flap}

In this section we further analyse the results we obtained in previous sections and explore the physical separation control mechanism. We first present the flow mechanism of a clean NACA0012 airfoil. Figure 10 shows the velocity contour of the fluid and streamlines of the flow around a NACA0012 airfoil with $\mathrm{AoA}=10^{\circ}$ and $R e=1000$. As shown in Figure 10a, there is a long clockwise vortex on the airfoil upper surface as reported elsewhere [44]. This clockwise vortex is termed a "bubble" in the rest of the present paper. The separation point is near the leading edge and the reattachment point is near the trailing edge. In this situation, the airfoil has the highest lift and drag (point A in Figure 5a). Then the long bubble moves towards the trailing edge and induces a small anticlockwise vortex there (Figure 10b), this induced vortex weakens the strength of bubble and causes the airfoil lift and drag to decrease (point B in Figure 5a). In Figure 10c, the induced vortex grows in size and strength at the airfoil trailing edge, and collapses the bubble further, causing the airfoil lift and drag to achieve their minimum values (point $C$ in Figure 5a). At point $D$ in Figure 5a, the airfoil lift and drag increase due to formation of new bubble with a single clockwise vortex trapped inside (Figure 10d). The cycle then repeats.

\subsection{Mechanism of separation control via a rigid flap}

Considering the optimal rigid flap case obtained in the previous section, we now explore the separation control mechanism behind it. Figure 11a and 11b show the velocity contours of the fluid and streamlines of flow around the airfoil with a rigid flap with position $P^{*}=0.9$, length $L^{*}=0.1$ and deployment angle $\theta=90^{\circ}$. It can be observed that, due to the obstruction of the flap, the separation point of the airfoil moves backward and the reattachment point moves forward, causing the present case to have a higher lift and smaller drag than the clean airfoil. The bubble on the airfoil upper surface is split into two parts by flap, one big bubble on the upstream side and one small bubble on the downstream side. When the induced vortex at the trailing edge grows, it only influences the downstream bubble, with the upstream bubble remaining unchanged. Hence, lift and drag fluctuations of this case are smaller than those of the clean airfoil. 


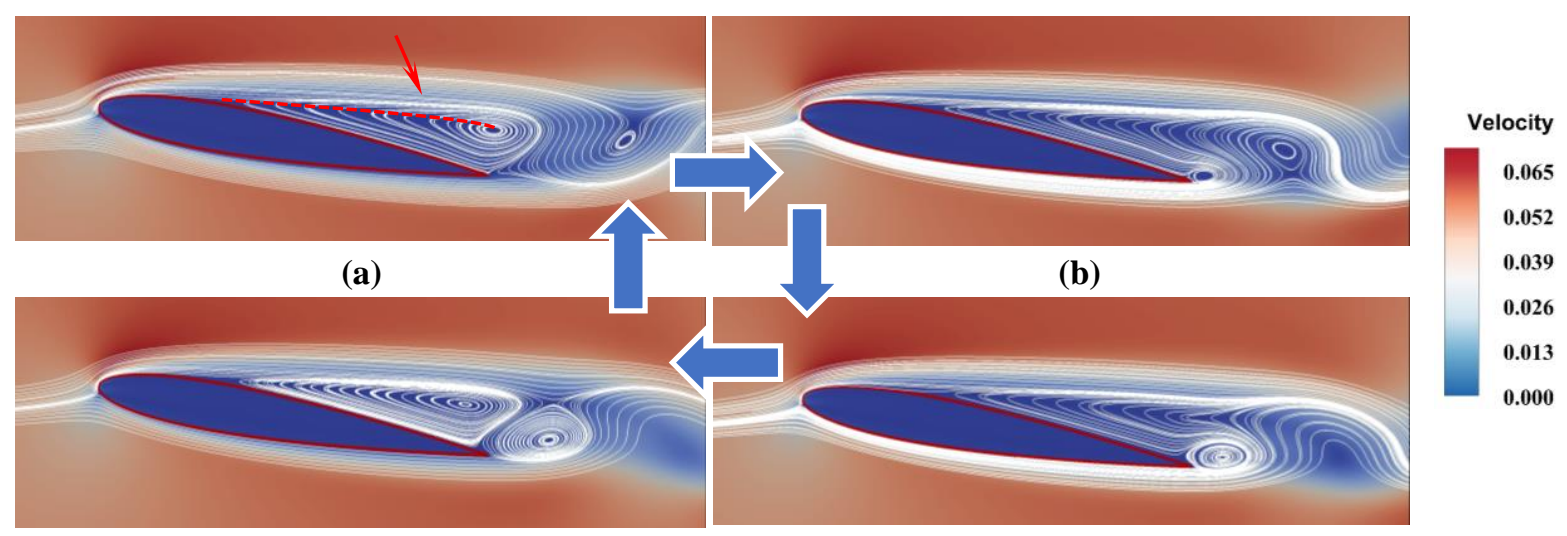

(d)

(c)

Figure 10: The fluid field velocity contour and streamline of flow around NACA0012 airfoil.

Figure $11 \mathrm{c}$ and $11 \mathrm{~d}$ show the time evolution of the mean lift and drag coefficients of the airfoil with flap, respectively. For comparison, the separate lift and drag coefficients of airfoil and the flap are also included. From these two figures, it can be seen that the lift coefficients of the airfoil and flap are in almost phase whereas their drag coefficients are almost completely out of phase. However, it should be noted that the drag coefficients of the flap are always negative, meaning that when the airfoil has maximum lift and drag coefficients, the flap also has maximum lift and drag coefficients but the drag acts in the opposite direction to the freestream.

Similar conclusions can also be obtained by analysing the streamlines in Figure 11a and 11b. As shown in Figure 11a, the small downstream bubble has little contact with the airfoil upper surface, while the rear part (from the flap position to the trailing edge) of airfoil is mainly affected by the induced vortex. From Figure 11b it can be seen that even though the induced vortex is anticlockwise, the vortex acting on the rear part of the airfoil is actually in a clockwise sense (as the red arrow pointed in Figure 11b) due to the obstructing flap. When the induced vortex is strongest, this local effect is most pronounced, at which point the lift and drag achieve maximum values (point A in Figure 11c and 11d). However, when the small downstream bubble exists, as shown in Figure 11a, it weakens the strength of induced vortex due to their opposing directions. When the small downstream bubble has highest strength, the rear vortex becomes anticlockwise (at the location indicated by the red arrow in Figure 11a), and the lift and drag of the airfoil achieve their minimum values (point B in Figure 11c and 11d).

As for the flap, because the strength of larger upstream bubble is much higher than the small downstream one, the flap is sucked forward by the big bubble, causing the negative drag values of the flap. In Figure 11a, the small bubble will counteract the suction of big bubble because they are on opposite sides of flap and have the same rotation directions. This causes the flap lift and drag to decrease. When the small bubble has highest strength, the flap lift and drag have minimum values (point D in Figure 11c and 11d). However, when the small bubble disappears (or it is too weak to be considered), as shown in Figure 11b, the flap is almost exclusively affected by the big bubble and its drag has a maximum value in the opposite direction of the freestream (point $\mathrm{C}$ in Figure 11c and 11d).

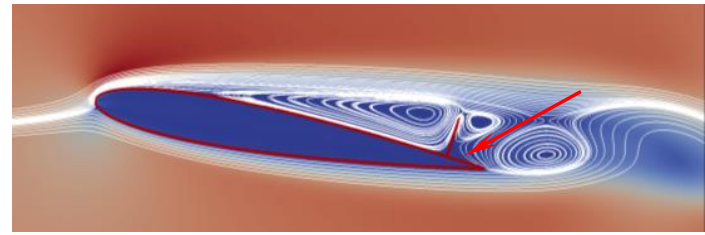

(a)

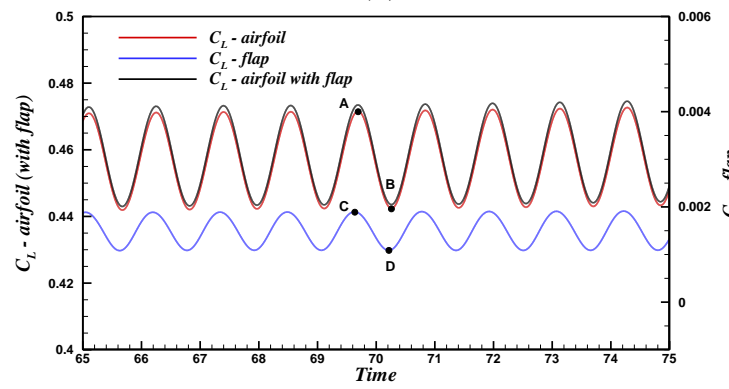

(c)

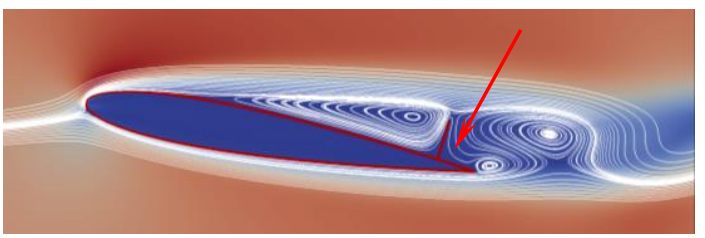

(b)

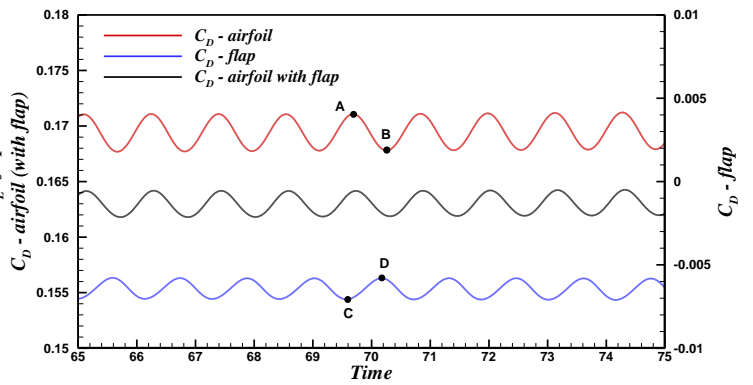

(d)

Figure 11: (a) and (b): Fluid field velocity contours and streamline of flow around airfoil with a rigid flap $\left(P^{*}=0.9 L^{*}=0.10 \theta=90^{\circ}\right)$. (c): Time evolution of lift coefficient of airfoil and (with) flap. (d): Time evolution of drag coefficient of airfoil and (with) flap. 


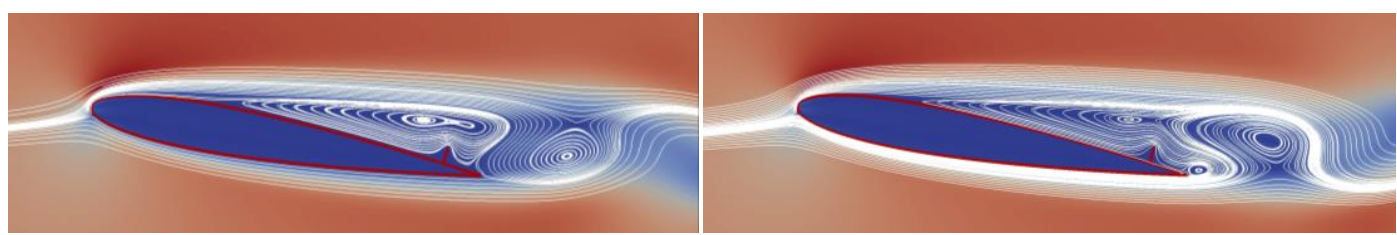

(a)

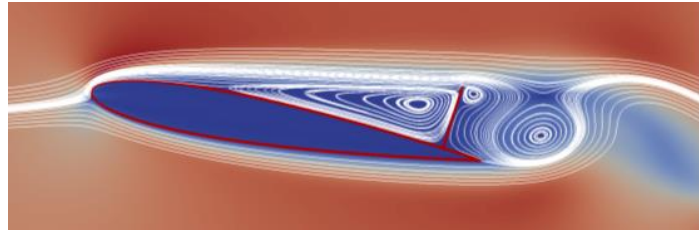

(c)

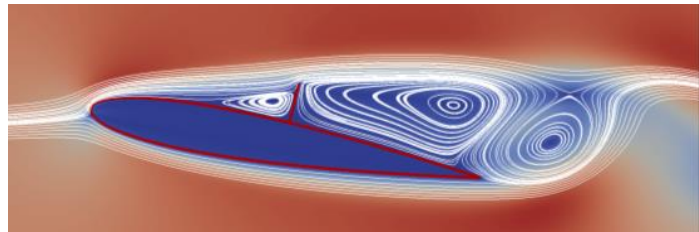

(e)

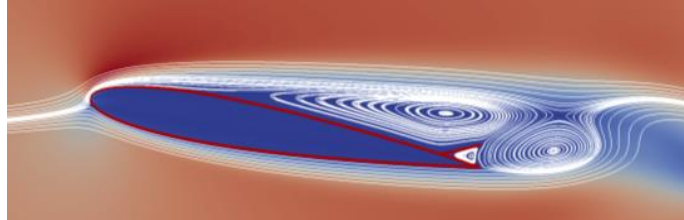

(g)

(b)

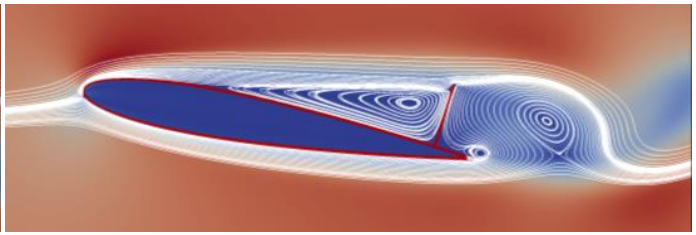

(d)

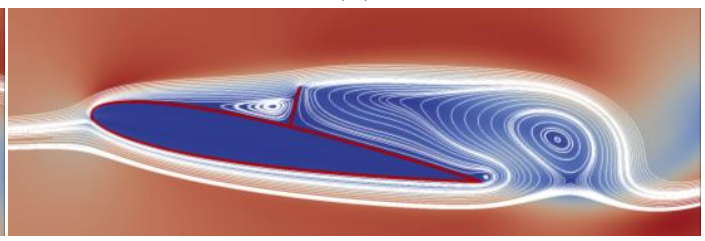

(f)
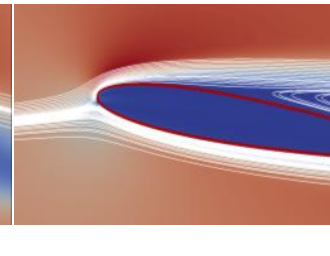

(h)

Figure 12: The fluid field velocity contour and streamline of flow around airfoil with a rigid flap for $(\mathbf{a}-\mathbf{b}): P^{*}=0.9 L *=0.04 \theta=90^{\circ}$. (c-d): $P^{*}=0.9 L^{*}=0.16 \theta=90^{\circ}$. (e-f): $P^{*}=0.5 L^{*}=0.1 \theta=90^{\circ}$. (g-h): $P^{*}=0.9 L^{*}=0.1 \theta=30^{\circ}$.

When the length of the flap is small, the corresponding velocity contours of the fluid and streamlines of flow around the airfoil are shown in Figure $12 \mathrm{a}$ and $12 \mathrm{~b}$ where the rigid flap has position ratio $P^{*}=0.9$, length ratio $L^{*}=0.04$ and deployment angle $\theta=90^{\circ}$. Similar to the previous case, the separation point of airfoil moves backward and the reattachment point moves forward because of the obstructing flap. This causes the present case to have higher lift and smaller drag than the clean airfoil. Again, it can be seen in Figure 12a and 12b that the bubble on the airfoil upper surface is split into two parts, one big upstream bubble and one small downstream bubble. However, because the flap is too short, these two bubbles do not separate entirely and still influence each other. When the induced vortex grows, it affects both of these two bubbles but mainly the small bubble. As the upstream bubble is only marginally affected, the lift and drag fluctuations is still smaller than the clean airfoil. It should be noted that because the flap has a smaller length than the previous case, its obstruction function is weaker and its effect on the aerodynamic performance of the airfoil is subsequently smaller.

When the flap has a longer length, the corresponding results are shown in Figure $12 \mathrm{c}$ and $12 \mathrm{~d}$ where the rigid flap has position ratio $P^{*}=0.9$, length ratio $L^{*}=0.16$ and deployment angle $\theta=90^{\circ}$. It can be observed from these two figures that a longer flap not only blocks the whole bubble on airfoil upper surface, but also blocks some non-recirculating flow from the airfoil leading edge. On the tip of flap, a small bubble is shed and this small bubble interacts with the induced vortex. As with Figure 11, this small downstream bubble has not direct action on the airfoil upper surface (Figure 12c). The rear part (from flap position to trailing edge) of the airfoil is mainly affected by the induced vortex. Even though the induced vortex is anticlockwise, because of the obstruction of flap, the vortex acting on the rear part of the airfoil is actually clockwise (Figure 12d). When the induced vortex becomes strongest, the rear part vortex is also strongest, making the lift and drag of the airfoil achieve its maximum values. As shown in Figure 12c, when the small bubble exists, it weakens the strength of induced vortex because they have different rotation directions, making the rear part vortex weak. When the small bubble is at its highest strength, the rear part vortex becomes anticlockwise。

When the flap moves forward to the airfoil leading edge, a new phenomenon is observed. Figure $12 \mathrm{e}$ and $12 \mathrm{f}$ show the results of the airfoil with the rigid flap which has position ratio $P^{*}=0.5$, length ratio $L^{*}=0.1$ and deployment angle $\theta=90^{\circ}$. It can be observed from these two figures that because the flap is close to the airfoil leading edge, even though it is not very long, it still blocks some flow from leading edge. A new bubble is generated on the airfoil rear part (from flap positon to trailing edge) and it is much bigger than the upstream bubble, this new bubble still acts on the rear part of the airfoil and is affected by the induced vortex. As shown in Figure 12e, when the upstream bubble and new downstream bubble cover the entire airfoil upper surface, the airfoil has a maximum lift and drag like the clean airfoil. When the induced vortex grows, as shown in Figure 12f, it weakens the strength of downstream bubble, and the airfoil lift and drag decreases. 
When the induced vortex becomes strongest, the airfoil has a minimum lift and drag.

Table 3: The corresponding values of distance ratio $S^{*}$ for different position ratio $P^{*}$

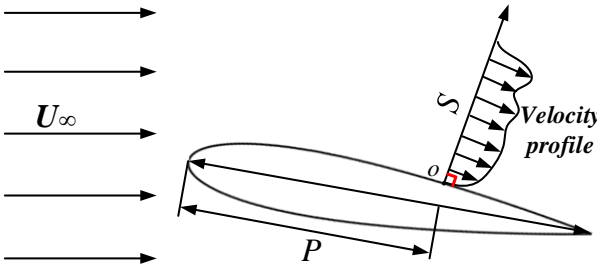

Figure 13: Velocity profile coordinate system

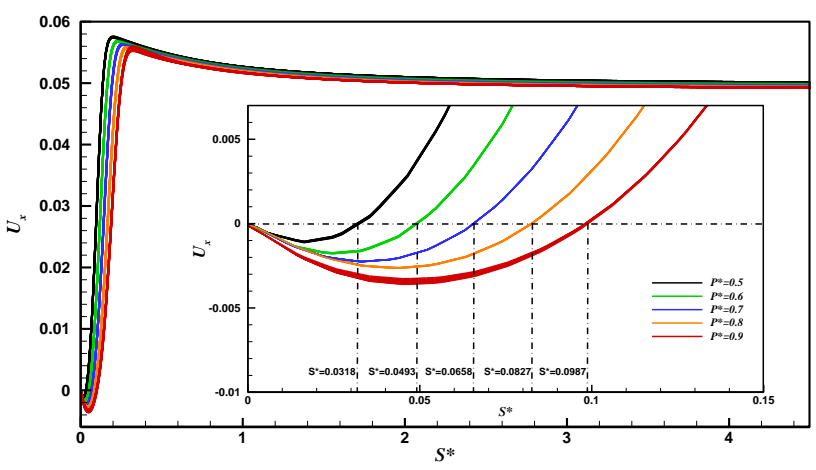

Figure 14: Distribution of velocity $U x$ in direction normal to airfoil upper surface in one period at different positions, with inset zoom.

When the flap has a smaller deployment angle, the corresponding results are shown in Figure $12 \mathrm{~g}$ and $12 \mathrm{~h}$ where the rigid flap has position ratio $P^{*}=0.9$, length ratio $L^{*}=0.1$ and deployment angle $\theta=30^{\circ}$. It can be observed in these two figures that the fluid velocity contours and streamlines are very similar to the results of the clean airfoil case, except for one more small bubble on the corner between the flap and the airfoil upper surface. Because the rear part of the airfoil is always covered by the corner bubble, we concentrate on its front part. As shown in Figure 12g, when the long bubble covers the whole front part, the airfoil has a maximum lift and drag coefficients. In Figure $12 \mathrm{~h}$, when the induced vortex grows, it weakens the strength of long bubble, causing the airfoil lift and drag to decrease. When the induced vortex becomes strongest, the airfoil has a minimum lift and drag.

Based on the above discussion, it is easy to see that when the flap tip is at the red dashed line shown in Figure 10a, it bisects the bubble on the airfoil upper surface but does not block the non-recirculating flow from airfoil leading edge. To obtain the exact position of the red dashed line in Figure 10a, we extract the velocity profile along the normal direction of airfoil upper surface. As shown in Figure 13, the distance from the flap root along the normal direction is $S$, and it is scaled by the airfoil chord $c$ as: $S^{*}=S / c$, where $S^{*}$ is called the distance ratio.

Figure 14 shows the distribution of velocity $U_{x}$ along normal direction of airfoil upper surface in one period at five different positions ( $P^{*}$ is from 0.5 to 0.9 ), it should be noted that each line in this figure actually represents a group of lines because the velocity profile always changes with time. From this figure, it can be seen that when the distance ratio $S^{*}$ equals zero, all five lines equal zero because of the no-slip boundary condition. As $S^{*}$ increases, these five lines decrease first to negative values, and then increase rapidly to a peak. As $S^{*}$ continues to increase, these lines begin decrease tending to a $x$ - lattice velocity of 0.05 , which is also the $x$ - lattice velocity of the freestream.

It can be seen that the red dashed line in Figure 10a is actually a line where velocity in the $x$ direction equals zero, so we draw a line $U_{x}=0$ in Figure 14 and obtain corresponding values of $S^{*}$, as shown in Table 3. Again, it should be noted that these values are approximations (or mean values) because the velocity profiles always change with time. The obtained distance ratio values are actually the optimal lengths of flap at five different positions, from Figure 6a and 6b, it can be seen that these values agree very well with the results we obtained in previous section.

\subsection{Mechanism of separation control via a flexible flap}

When the flap becomes flexible, as discussed in the Section 3.3, the flap has two flapping patterns: flapping on one side and flapping on both sides of its initial position. To analyse the first flapping pattern, we choose the optimal flexible flap case obtained in previous section and explore the separation control mechanism behind it. Figure 15 shows the results of the airfoil with a flexible flap which has position ratio $P^{*}=0.9$, length ratio $L^{*}=0.1$, deployment angle $\theta=90^{\circ}$, bending coefficient $K^{*}=0.08$ and mass ratio $M^{*}=15$.

As discussed in Figure 11, when the flap is rigid, the drag of the flap is always negative because of the suction of upstream bubble. Now the flap becomes flexible and its tip is sucked by the upstream bubble and moves forward, as shown in Figure 15a.This motion suppresses the upstream bubble and make the separation point move backward further, so the present case has better aerodynamic performance than the rigid case. On the other hand, this forward motion causes 


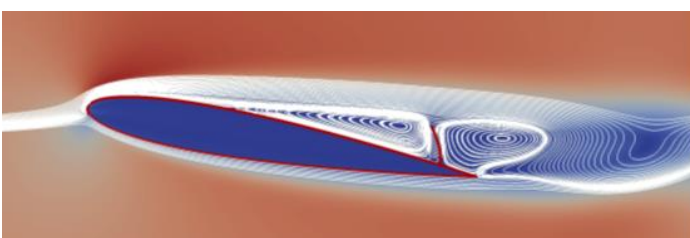

(a)

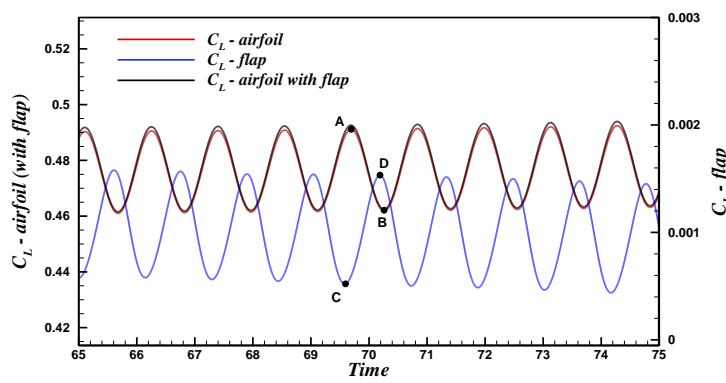

(c)

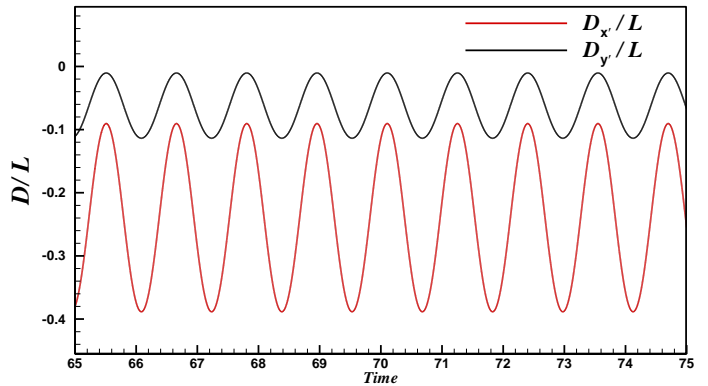

(e)

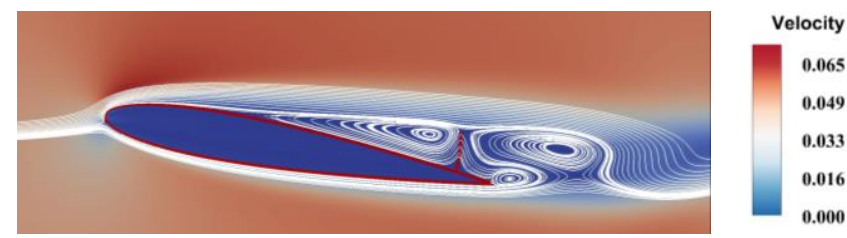

(b)

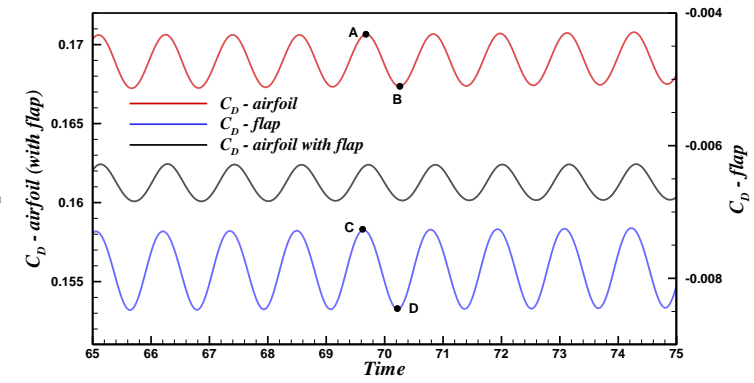

(d)

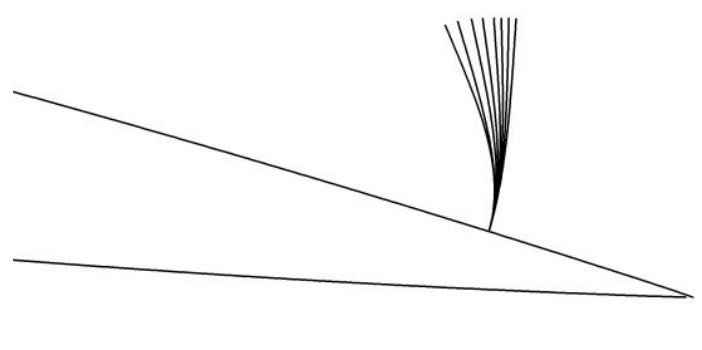

(f)

Figure 15: (a) and (b): The fluid field velocity contour and streamline of flow around airfoil with a flexible flap $\left(P^{*}=0.9 L^{*}=0.1\right.$ $\left.\theta=90^{\circ} K^{*}=0.08 M^{*}=15\right)$. (c): Time evolution of lift and (d): drag coefficient of airfoil and (with) flap. (e): Time evolution of $x^{\prime}-$ and $y$ '-displacements of flap tip. (f): Superposition of the flap positions.

a small gap between the upstream bubble and flap, decreasing the suction of the upstream bubble. When the flap tip reaches its left limit position, the suction of the upstream bubble becomes weakest while the suction of downstream bubble becomes strongest, causing the flap tip to flap back. As shown in Figure 15b, because of the effect of induced vortex, the strength of the downstream bubble is weakened, reducing the suction action. When the flap tip reaches its flap limit position, the flap is sucked by the upstream bubble and flaps back again. The time evolution of $x$ '- and $y$ '-displacements of the flap tip and the superposition of flap positions are shown in Figure 15e and 15f, respectively. It should be noted that in Figure 15a and 15b some streamlines cross through the flap, it is a normal phenomenon associated with the soft interface of the immersed boundary method.

In Figure 15c and 15d, it can be seen that the lift coefficients of the airfoil and flap are out of phase while the drag coefficients of are in phase. However, it should be noted that the flap drag coefficients are always negative, which means when the airfoil has a maximum drag coefficient, the flap actually has a minimum drag coefficient in the opposite direction to the freestream.

For the airfoil, as shown in Figure 15a, when the upstream bubble and downstream bubble both cover the upper surface, the airfoil has a maximum lift and drag coefficient (point A in Figure 15c and 15d). In Figure 15b, when the induced vortex grows, it weakens the strength of the long bubble, causing the airfoil lift and drag to decrease. When the induced vortex becomes strongest, the airfoil has a minimum lift and drag (point B in Figure 15c and 15d).

As for the flap, because the strength of the upstream bubble is always higher than the downstream bubble, as shown in Figure 15a, at its left flap limit position, the downstream bubble has its maximum strength and will counteract the suction of the upstream bubble. This causes the flap drag to decrease. When the downstream bubble has its highest strength, the flap has its minimum drag (point $\mathrm{C}$ in Figure 15d). However, when the downstream is weakened by the induced vortex, as shown in Figure 15b, the flap is mainly affected by the upstream bubble, and its drag has its maximum value but in the opposite direction to the freestream (point D in Figure 15d). 


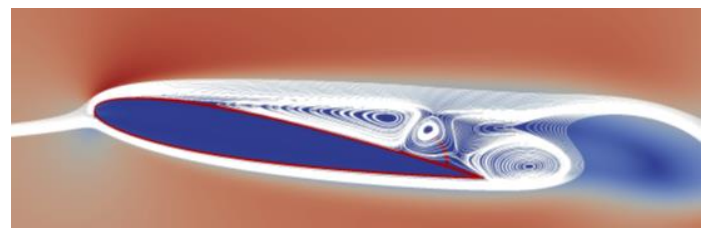

(a)

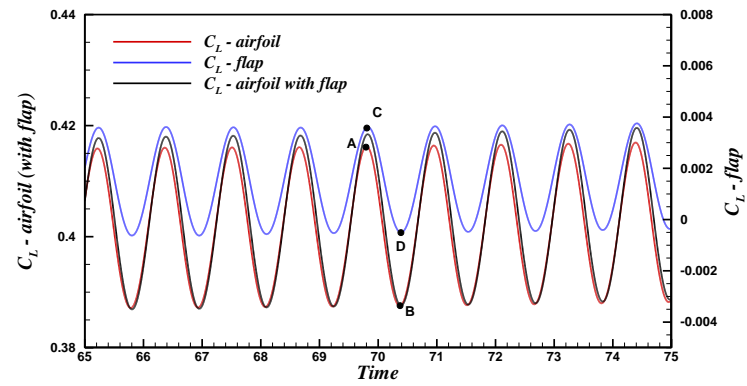

(c)

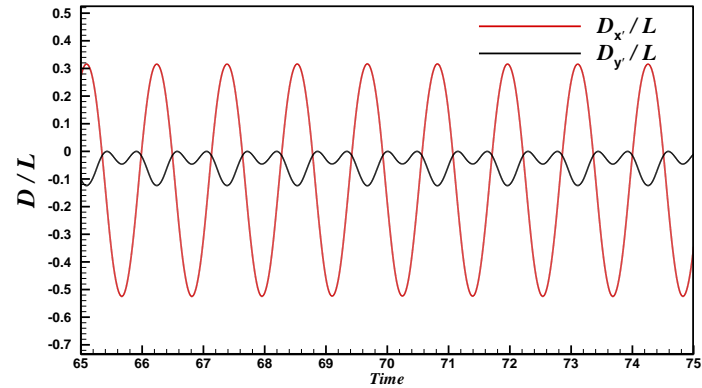

(e)

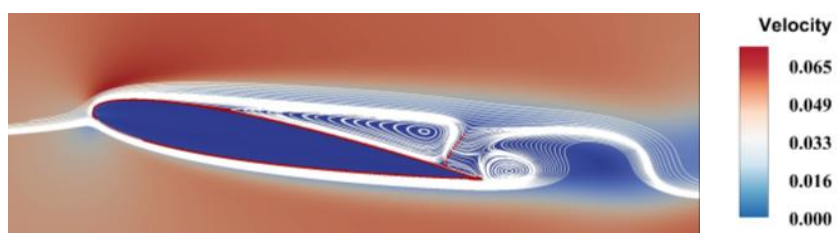

(b)

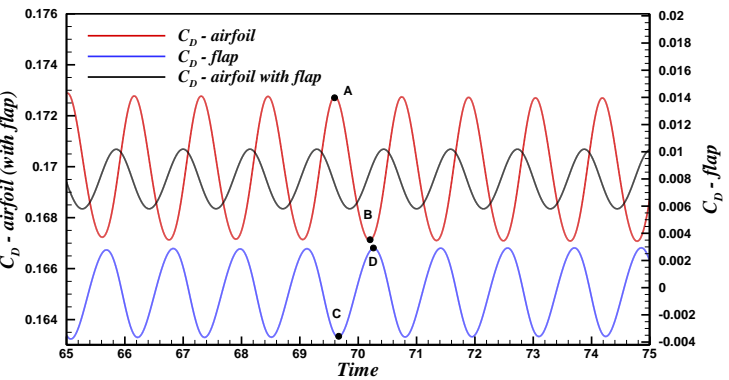

(d)

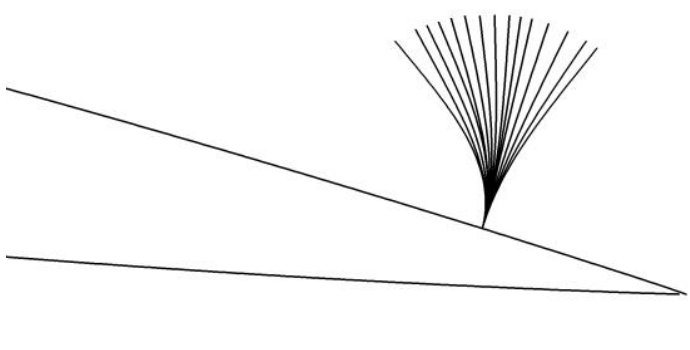

(f)

Figure 16: (a) and (b): The fluid field velocity contour and streamline of flow around airfoil with a flexible flap $\left(P^{*}=0.9 L^{*}=0.1\right.$ $\left.\theta=90^{\circ} K^{*}=0.01 M^{*}=50\right)$. (c): Time evolution of lift and (d): drag coefficient of airfoil and (with) flap. (e): Time evolution of $x^{\prime}-$ and $y^{\prime}$-displacements of flap tip. (f): Superposition of the flap positions.

When the flap becomes more rigid, it will have smaller deformations. The fluid field velocity contour and streamlines are similar to the previous case. However, when the flap is more flexible, it transitions to a different flapping pattern. Now we choose the least optimal flexible flap case obtained in the previous section and explore the separation control mechanism behind it. Figure 16 shows the results of the airfoil with the flexible flap which has position ratio $P^{*}=0.9$, length ratio $L^{*}=0.1$, deployment angle $\theta=90^{\circ}$, bending coefficient $K^{*}=0.01$ and mass ratio $M^{*}=50$. As shown in Figure 16a, when the flap is sucked by the upstream bubble and flaps forward, there is an anticlockwise vortex generated between the upstream bubble and flap. This new vortex pushes the flap tip back. In Figure 16b, when the vortex sheds from the airfoil trailing edge, the flap is sucked by the upstream bubble and flaps back again. The time evolution of $x$ '- and $y$ 'displacements of the flap tip and the superposition of flap positions are shown in Figure 16e and 16f, respectively.

In Figure 16c and 16d, it can be seen that the lift coefficients of the airfoil and the flap are in phase while the drag coefficients are out of phase. However, it should be noted that when the airfoil has maximum drag coefficients, the flap drag coefficients are negative, which means the flap drag acts in the opposite direction to the freestream. For the airfoil, as shown in Figure 16a, when the new generated vortex acts on the airfoil upper surface, the airfoil has minimum lift and drag coefficients (point B in Figure 16c and 16d). As the new vortex moves forward, the upstream bubble extends its region of influence and the airfoil lift and drag increases. In Figure 16b, when a new vortex is shed from airfoil trailing edge, the airfoil has its maximum lift and drag coefficients (point A in Figure 16c and 16d).

As for the flap, as shown in Figure 16a, when the new generated vortex exists, the flap is pushed backward by it because it is anticlockwise, and the flap drag acts in the freestream direction. When the new vortex becomes strongest, the flap has its maximum lift and drag coefficients (point D in Figure 16c and 16d). In Figure 16b, when the new vortex moves to the airfoil trailing edge, the flap drag is decreased. Finally, when the new vortex is shed from airfoil trailing edge, the flap is sucked by the upstream bubble, and it has its maximum drag coefficient in the opposite direction to the freestream (point $\mathrm{C}$ in Figure 16d).

\section{Conclusions}

We have combined the lattice Boltzmann, immersed boundary and finite element methods to investigate the separation control of a NACA0012 airfoil via a rigid/flexible flap at $R e=1000$ and $\mathrm{AoA}=10^{\circ}$. For the rigid flap, the aerodynamic 
performance of airfoil together with flap which has various lengths, attachment positions and deployment angles are investigated, and the positive and negative effects of the flap under different configurations are observed. The results show that when the rigid flap has a length ratio $L^{*}=0.1$, position ratio $P^{*}=0.9$ and deployment angle $\theta=90^{\circ}$, the airfoil with flap achieves optimal aerodynamic performance. Compared to the clean airfoil, the mean lift coefficient of this optimal case is improved by $9.48 \%$, the mean drag coefficient is decreased by $2.55 \%$, the mean lift-drag ratio is increased by $12.34 \%$, the maximum lift fluctuation is decreased by $40.54 \%$ and the maximum drag fluctuation is decreased by $56.65 \%$.

Under this optimal configuration, the aerodynamic performance of the airfoil together with flexible flap which has various bending coefficients and mass ratios are investigated, and the positive and negative effects of the flap with different material properties are obtained. Two flapping patterns of flap are observed: flapping on one side of its initial position and flapping on both sides of its initial position. The results show that when the flexible flap has a bending coefficient $K^{*}=0.08$ and a mass ratio $M^{*}=15$, the airfoil with flap achieves optimal aerodynamic performance. Compared to the clean airfoil case, the mean lift coefficient of this optimal case is improved by $13.51 \%$, the mean drag coefficient is decreased by $3.67 \%$, the mean lift-drag ratio is improved by $17.84 \%$, the maximum lift fluctuation is decreased by $40.90 \%$ and the maximum drag fluctuation is decreased by $56.90 \%$.

Analysis of the physical mechanism shows that under certain configurations, the flap can divide the bubble on the airfoil upper surface and make the separation point of airfoil move backward, which has a positive effect on aerodynamic performance. However, when the flap is too long or too close to the airfoil leading edge, it will also block non-recirculating flow from the from airfoil leading edge causing a negative effect on aerodynamic performance. Based on this conclusion, the optimal length of flap at different positions along the upper surface are obtained and reported. For the flexible flap, when its deformation is not large, the forward motion of its tip suppresses the upstream bubble and makes the separation point move backward further, which has a positive effect on performance. However, if the flap has large deformation, a new anticlockwise vortex on the airfoil upper surface is generated. This new vortex has a negative effect on performance compared to the clean airfoil case.

The obtained results in this paper can provide meaningful references for engineering problems, and this biologically inspired passive separation control device has a real potential to be applied to realistic MAV designs due to its simplicity. Owing to the universality of the algorithm used in this paper, in the future, the present work will be easily to be extended to three-dimensional simulations such as a wing with a rigid/flexible plate attached or an array of flaps.

\section{Acknowledgements}

The present work was partially supported by the Fundamental Research Funds for the Central Universities of Northwestern Polytechnical University, NSFC Foundation (Grant No.1167225, No.11511130053) and the Natural Science Foundation of Shaanxi Provence (No.2016JM1007).

\section{References}

1. Schluter, Jorg U. "Lift Enhancement at Low Reynolds Numbers Using Self-Activated Movable Flaps." Journal of Aircraft 47.1(2012):348-351.

2. Pines, Darryll J., and F. Bohorquez. "Challenges Facing Future Micro-Air-Vehicle Development." Journal of Aircraft 43.2(2006):290-305.

3. Bechert D, Bruse M, Hage W, et al. Biological surfaces and their technological application - Laboratory and flight experiments on drag reduction and separation control[C] AIAA Shear Flow Conference, 29.6.1997-2.7.1997, Snowmass Village, Co, Usa. 1997.

4. Liebe, W. "Der auftrieb am tragflügel: Entstehung und zusammenbruch." Aerokurier 12.1520 (1979): 54.

5. Carruthers, Anna C., Adrian LR Thomas, and Graham K. Taylor. "Automatic aeroelastic devices in the wings of a steppe eagle Aquila nipalensis." Journal of Experimental Biology 210.23 (2007): 4136-4149.

6. $\quad$ Meyer, Robert, et al. "Separation Control by Self-Activated Movable Flaps." Aiaa Journal Vol. 45.1(2007):191-199.

7. Inaoka K, Mori T, Yamaguchi M , et al. Feedback flow control of a low-Re airfoil by flap actuators[J]. Journal of Fluids \& Structures, 2015, 58:319-330.

8. Bechert, D. W., W. Hage, and R. Meyer. Self-actuating flaps on bird- and aircraft-wings. Flow Phenomena in Nature Volume 2: Inspiration, Learning and Application. DLR, 2006.

9. C H John Wang, Schlüter J. Stall control with feathers: Self-activated flaps on finite wings at low Reynolds numbers[J]. Comptes rendus - Mécanique, 2012, 340(1):57-66.

10. Gograve, T. Bramesfeld, and M. D. Maughmer. "Experimental Investigation of Self-Actuating, Upper-Surface, High-LiftEnhancing Effectors." Journal of Aircraft 39.1(2002):120-124.

11. Johnston, Joe, Ashok Gopalarathnam, and Jack Edwards. "Experimental investigation of bio-inspired high lift effectors on a $2-$ D airfoil." 29th AIAA Applied Aerodynamics Conference. (2011): 255-265 
12. Johnston, Joe, and Ashok Gopalarathnam. "Investigation of a bio-inspired lift-enhancing effector on a 2D airfoil." Bioinspiration \& biomimetics 7.3 (2012): 036003.

13. Brücker, Christoph, and Christoph Weidner. "Influence of self-adaptive hairy flaps on the stall delay of an airfoil in ramp-up motion." Journal of Fluids and Structures 47 (2014): 31-40.

14. Favier, Julien, et al. "Passive separation control using a self-adaptive hairy coating." Journal of Fluid Mechanics 627 (2009): 451-483.

15. Venkataraman, Divya, and Alessandro Bottaro. "Numerical modeling of flow control on a symmetric airfoil via a porous, compliant coating." Physics of Fluids 24.9 (2012): 093601.

16. Rosti M E, Omidyeganeh M, Pinelli A. Passive control of the flow around unsteady airfoils using a self-activated deployable flap[J]. Journal of Turbulence, 2017:1-25.

17. Rosti M E, Kamps L, Bruecker C , et al. The PELskin project-part V: towards the control of the flow around aerofoils at high angle of attack using a self-activated deployable flap[J]. Meccanica, 2017, 52(8):1811-1824.

18. Rosti, Marco Edoardo, M. Omidyeganeh, and A. Pinelli. "Study of flow around NACA0020 airfoil with hairy flaps during ramp-up motion." European Drag Reduction and Flow Control Meeting - Edrfcm 2015.

19. Harwood, Adrian R. G., et al. "LUMA: A many-core, Fluid-Structure Interaction solver based on the Lattice-Boltzmann Method." Softwarex 7(2018):88-94.

20. Zhu L, He G, Wang S, et al. An immersed boundary method based on the lattice Boltzmann approach in three dimensions, with application [J]. Computers and Mathematics with Applications, 2011, 61(12):3506-3518.

21. Cheng Y, Zhang H. Immersed boundary method and lattice Boltzmann method coupled FSI simulation of mitral leaflet flow [J]. Computers and Fluids, 2010, 39(5):871-881.

22. Fang-Bao T. Deformation of a Capsule in a Power-Law Shear Flow [J]. Computational and Mathematical Methods in Medicine, 2016, 2016:1-9.

23. Sui Y, Chew Y T, Roy P, et al. A hybrid method to study flow-induced deformation of three-dimensional capsules [J]. Journal of Computational Physics, 2008, 227(12):6351-6371.

24. Favier J , Revell A, Pinelli A . A Lattice Boltzmann-Immersed Boundary method to simulate the fluid interaction with moving and slender flexible objects[J]. Journal of Computational Physics, 2014, 261:145-161.

25. Uddin E , Huang W X, Sung H J . Actively flapping tandem flexible flags in a viscous flow [J]. Journal of Fluid Mechanics, 2015, 780:120-142.

26. Lincheng X, Fang-Bao T , John Y , et al. A novel geometry-adaptive Cartesian grid based immersed boundary-lattice Boltzmann method for fluid-structure interactions at moderate and high Reynolds numbers $[\mathrm{J}]$. Journal of Computational Physics, 2018:S0021999118305473.

27. Tullio D, Palma P D , Iaccarino G, et al. An immersed boundary method for compressible flows using local grid refinement [J]. Journal of Computational Physics, 2007, 225(2):2098-2117.

28. Felippa, C. A., \& Haugen, B. A unified formulation of small-strain corotational finite elements: i. theory. Computer Methods in Applied Mechanics \& Engineering, (2005), 194(21-24), 2285-2335.

29. Gong C, Fang Z, Chen G. A Lattice Boltzmann-Immersed Boundary-Finite Element Method for Nonlinear Fluid-Solid Interaction Simulation with Moving Objects [J]. International Journal of Computational Methods, 2018, 15(07): 1850063.

30. De Rosis A , Falcucci G , Ubertini S , et al. Aeroelastic study of flexible flapping wings by a coupled lattice Boltzmann-finite element approach with immersed boundary method[J]. Journal of Fluids and Structures, 2014, 49:516-533.

31. Succi S. The lattice Boltzmann equation: for fluid dynamics and beyond[M]. Oxford university press, 2001.

32. Bhatnagar, P. L., E. P. Gross, and M. Krook. "A Model for Collision Processes in Gases. I. Small Amplitude Processes in Charged and Neutral One-Component Systems." Phys Rev 94.3(1954):511--525.

33. Qian, Y. H., D. D'Humières, and P. Lallemand. "Lattice BGK Models for Navier-Stokes Equation." Epl 17.6BIS(2007):479.

34. Guo, Z., C. Zheng, and B. Shi. "Discrete lattice effects on the forcing term in the lattice Boltzmann method. " Physical Review E Statistical Nonlinear \& Soft Matter Physics 65.4 Pt 2B(2002):046308.

35. Doyle J F. Nonlinear analysis of thin-walled structures: statics, dynamics, and stability[M]. Springer Science \& Business Media, 2013.

36. Newmark, N. M. "A Method of Computation for Structural Dynamics." Proc Asce 85.1(1959):67-94.

37. Peskin, Charles S. "Flow patterns around heart valves: A numerical method." Journal of Computational Physics 10.2(1972):252-271.

38. Peskin, Charles S. The immersed boundary method[J]. Acta Numerica, 2002, 11:479-517.

39. Roma A M , Peskin C S , Berger M J . An Adaptive Version of the Immersed Boundary Method[J]. Journal of Computational Physics, 1999, 153(2):509-534.

40. Li Z, Favier J , D'Ortona U , et al. An immersed boundary-lattice Boltzmann method for single- and multi-component fluid flows[J]. Journal of Computational Physics, 2016, 304(C):424-440.

41. Wu J , Shu C . Implicit velocity correction-based immersed boundary-lattice Boltzmann method and its applications[J]. Journal of Computational Physics, 2009, 228(6):1963-1979.

42. Falagkaris, E. J., et al. "PROTEUS: A coupled iterative force-correction immersed-boundary multi-domain cascaded lattice Boltzmann solver." Computers \& Mathematics with Applications 74.10 (2017): 2348-2368.

43. Mittal S, Tezduyar T E. Massively parallel finite element computation of incompressible flows involving fluid-body interactions [J]. Computer Methods in Applied Mechanics \& Engineering, 1994, 112(1 - 4):253-282. 
44. Murthy P S, Holla V S, Kamath H. Unsteady Navier-Stokes solutions for a NACA 0012 airfoil[J]. Computer Methods in Applied Mechanics \& Engineering, 2000, 186(1):85-99.

45. Rechenberg, I., Bannasch, R., Patone, G. \& Müller, W., Aeroflexible Oberflächenklappen als 'Rückstrombremsen' nach dem Vorbild der Deckfedern des Vogelflügels, Statusbericht 1995 für das BMBF-Vorhaben 13N6536, Inst. f. Bionik u. Evolutionstechnik, TU Berlin, 1995. 


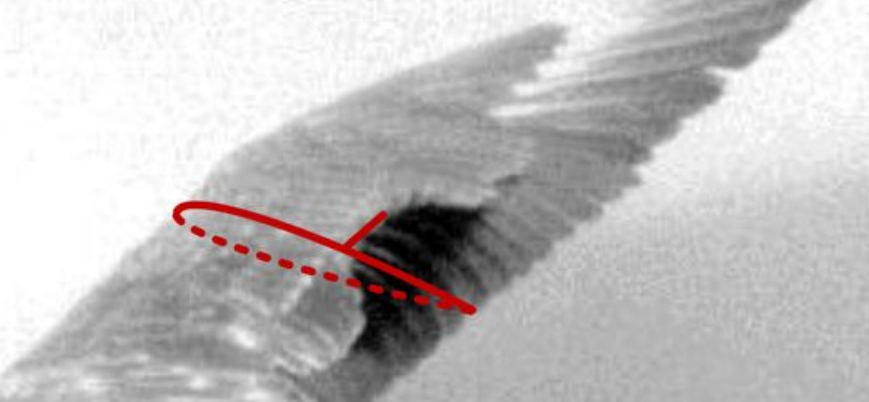
-

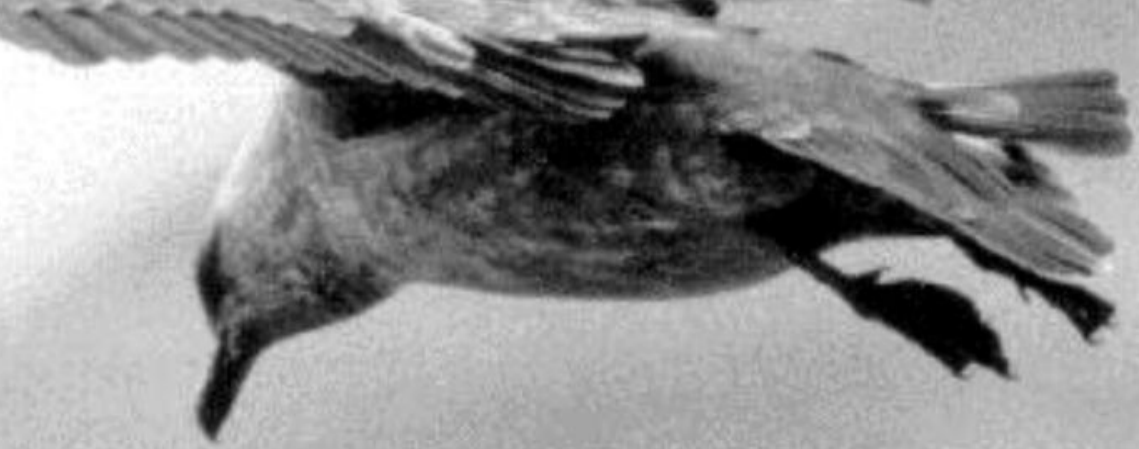




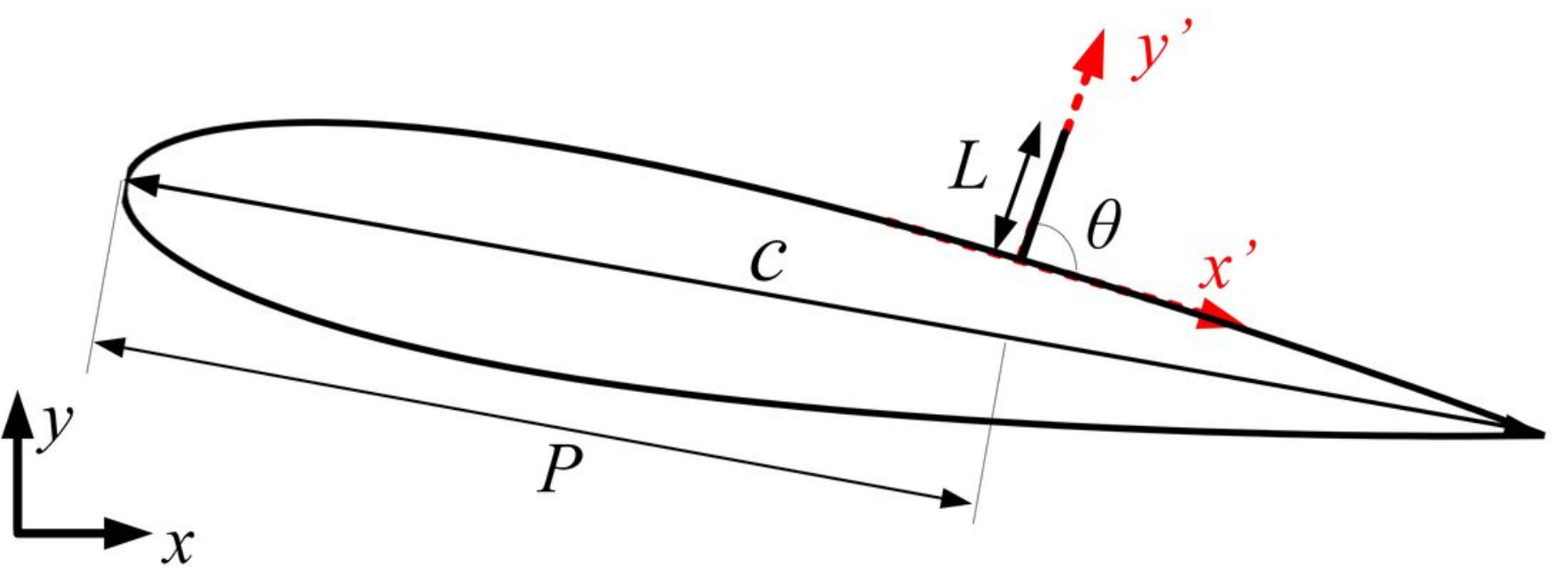




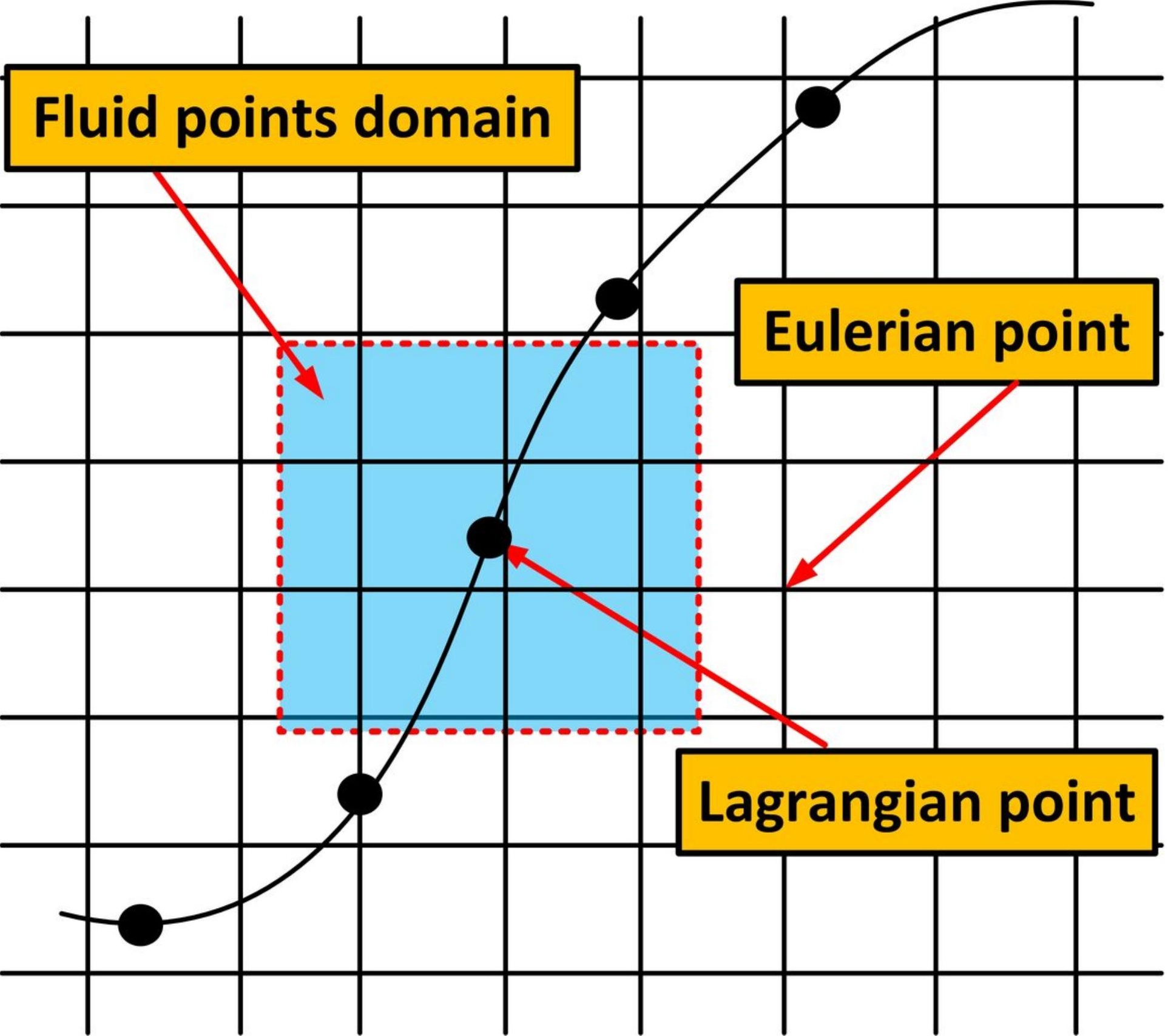




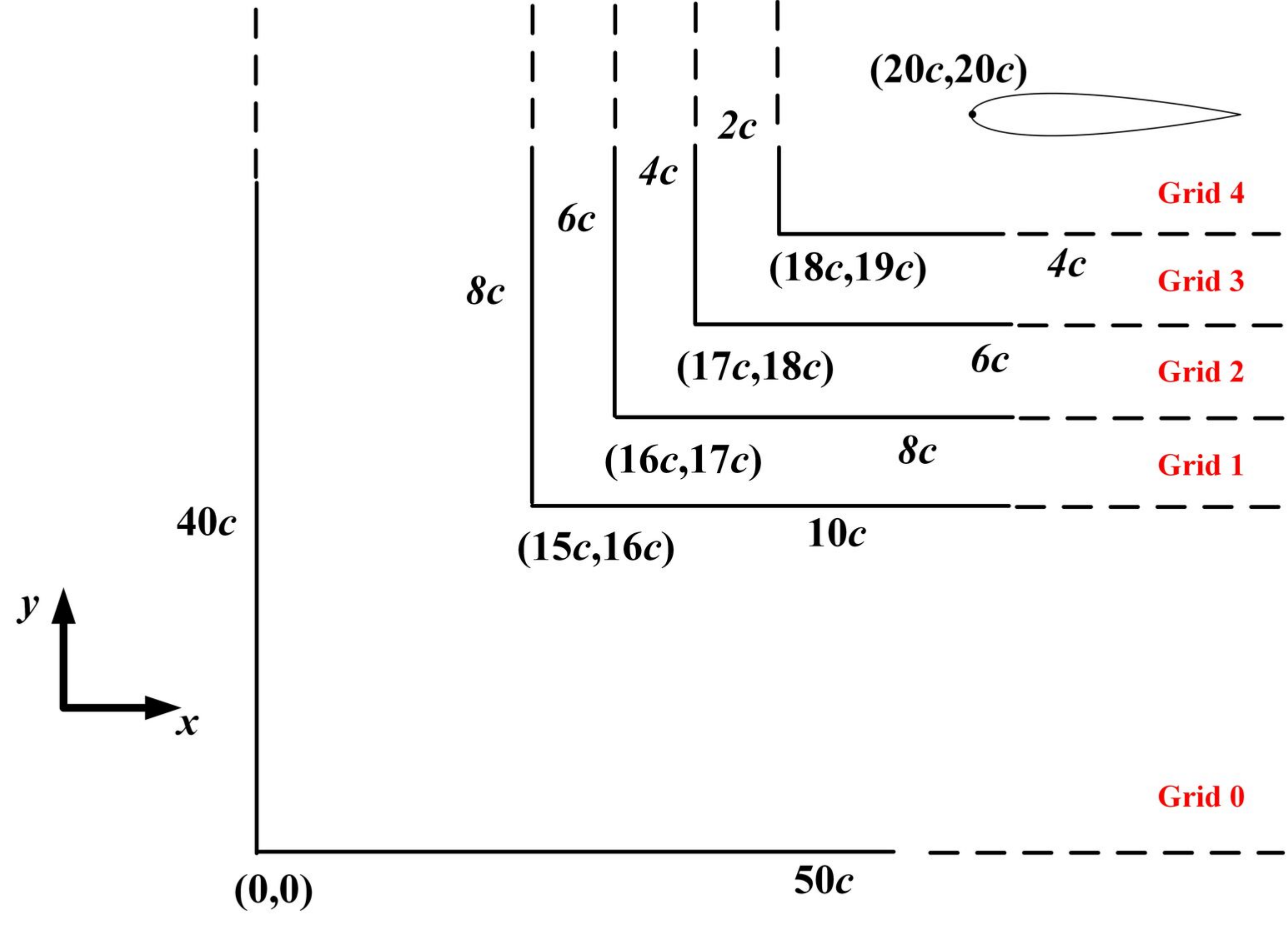




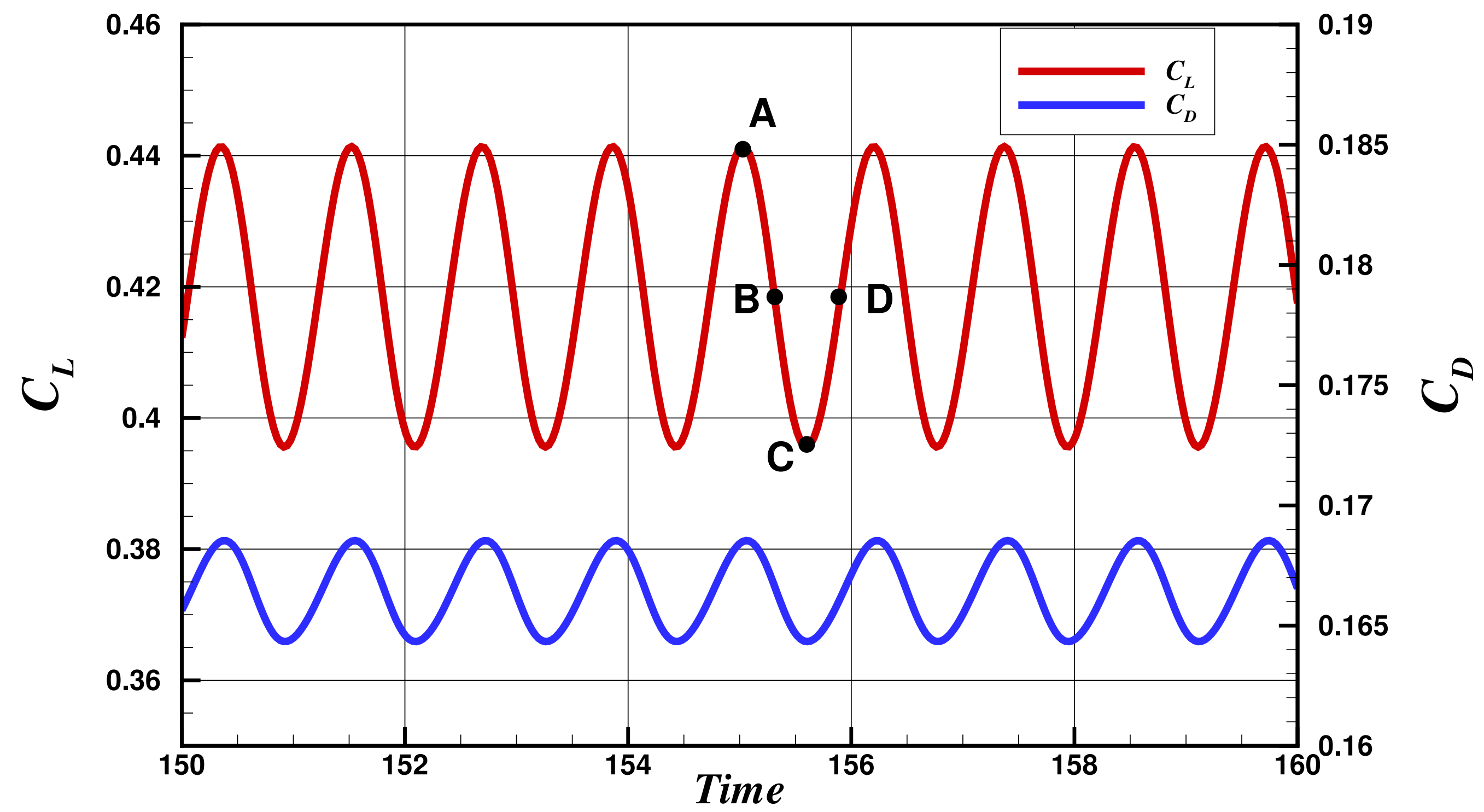




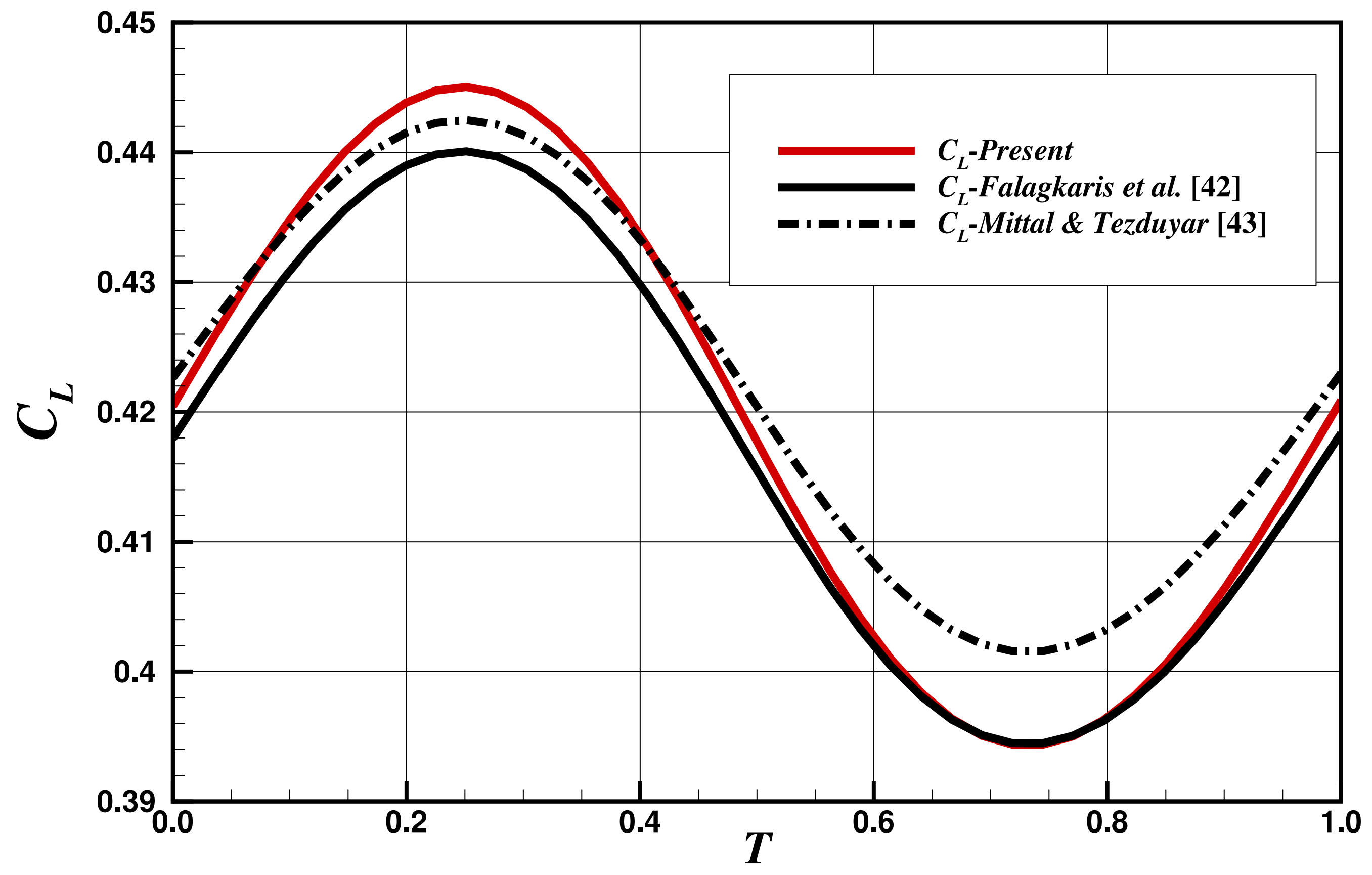




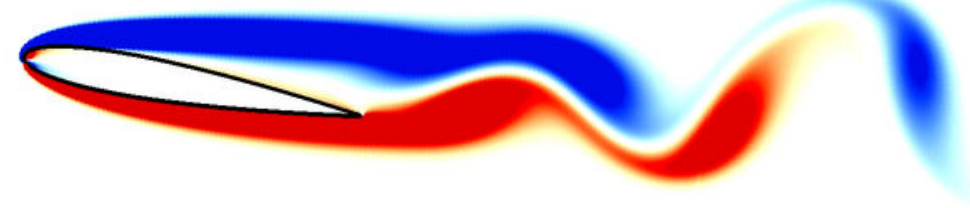


Vorticity

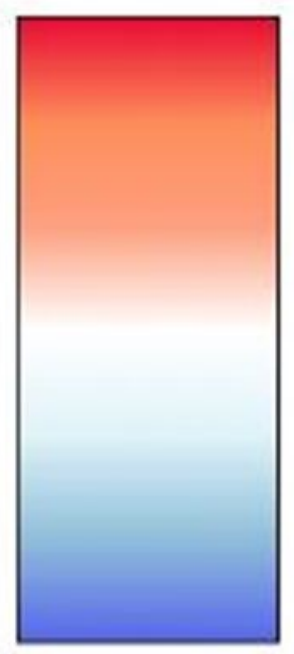

0.30

0.10

$-0.10$

$-0.30$ 


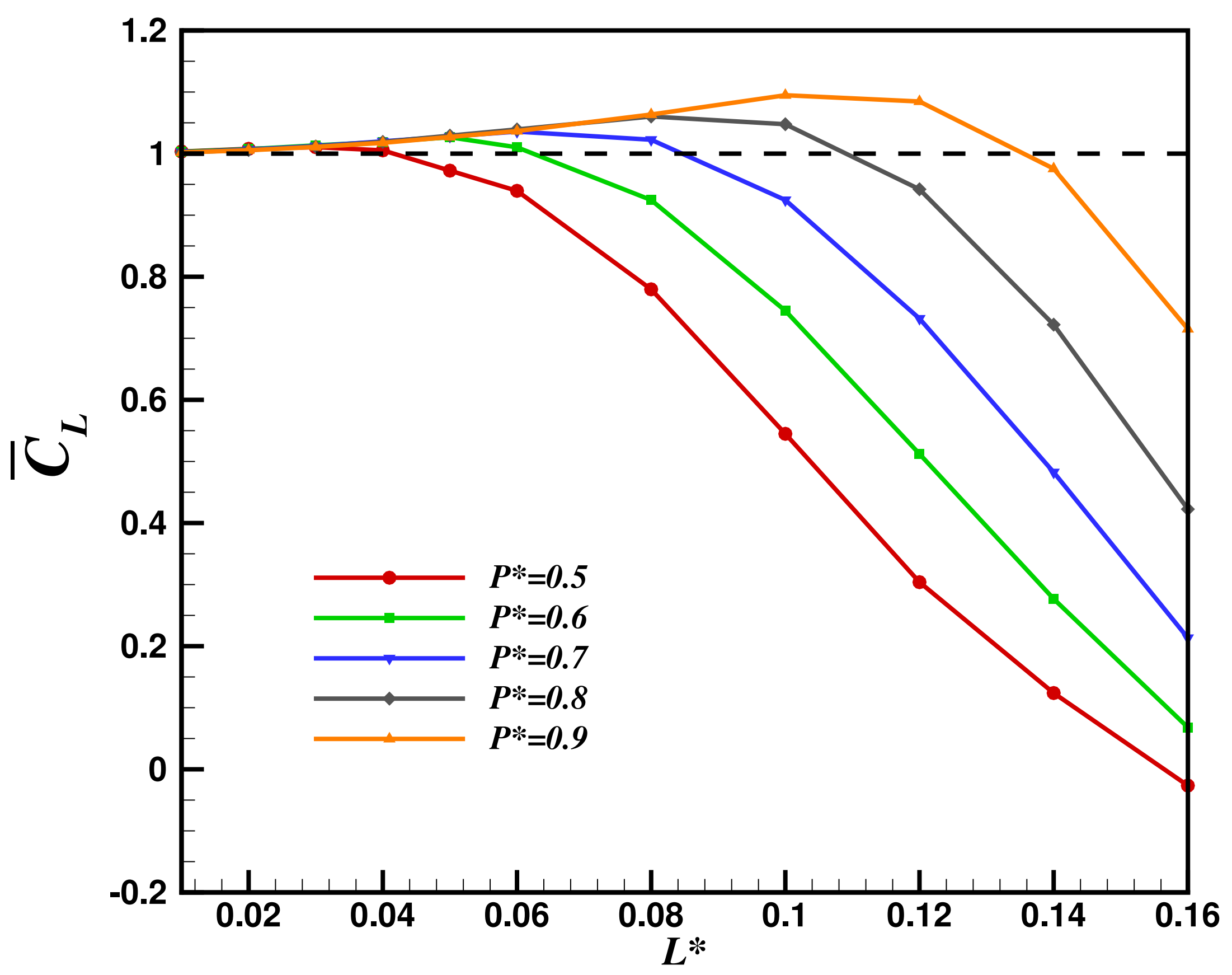




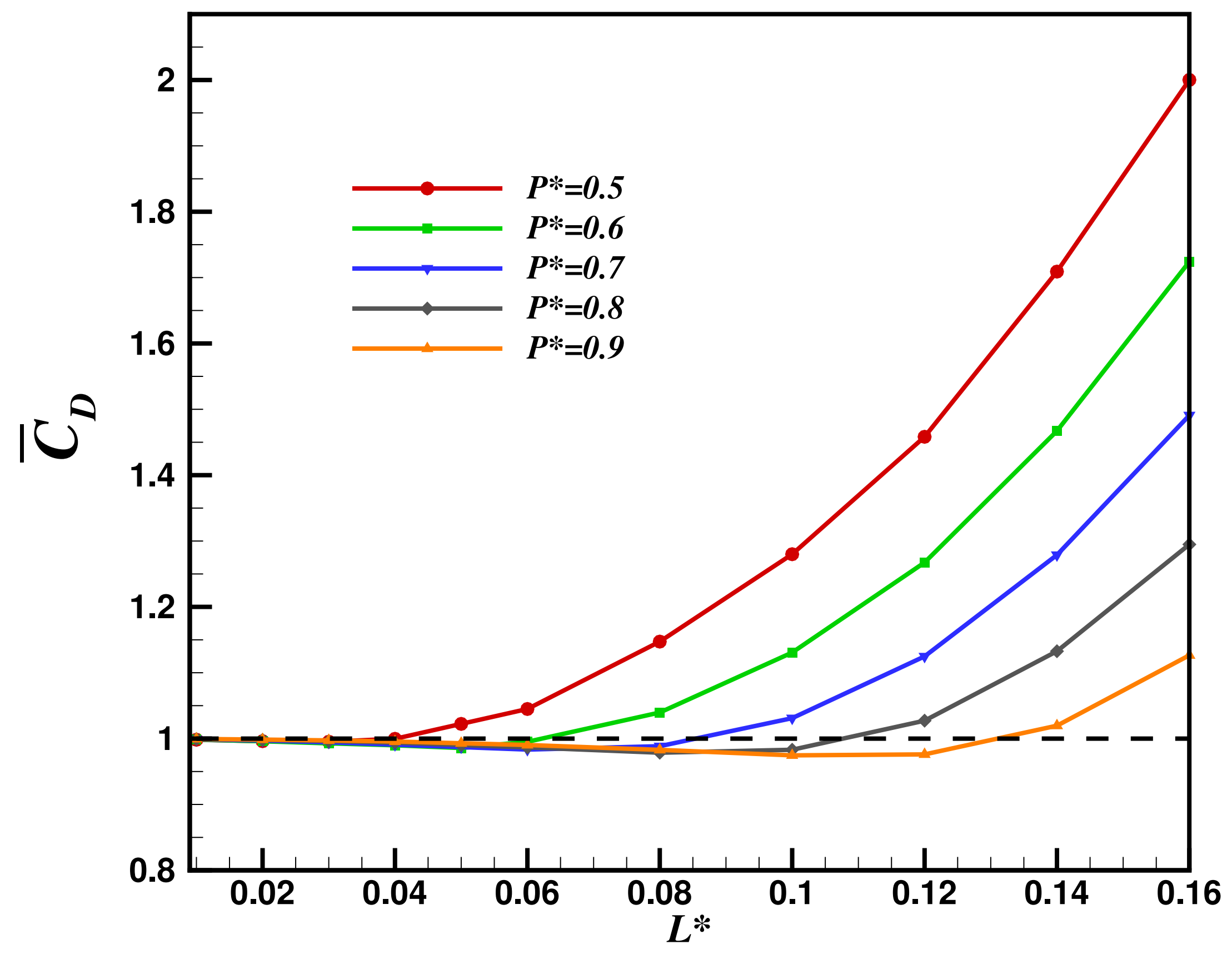




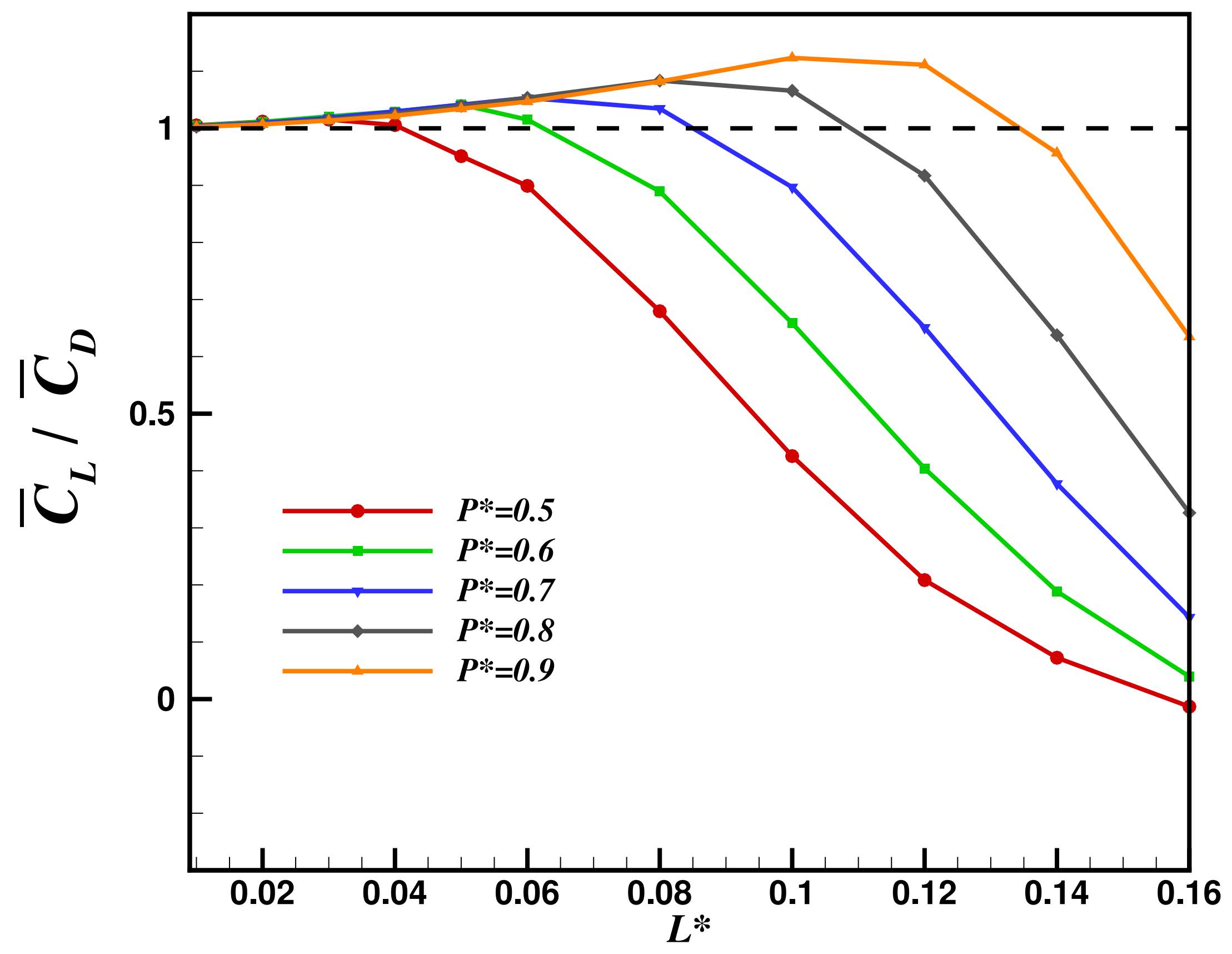




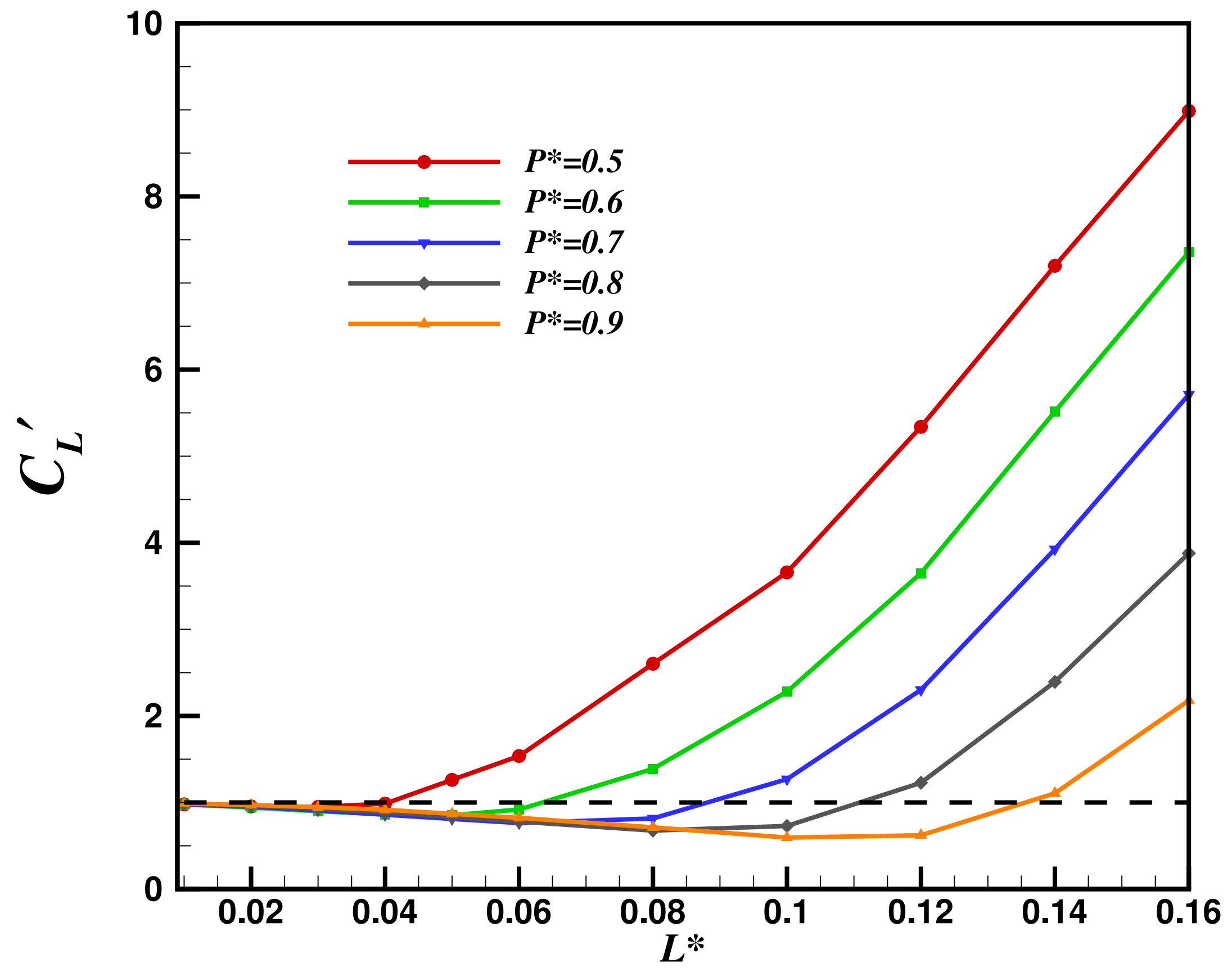




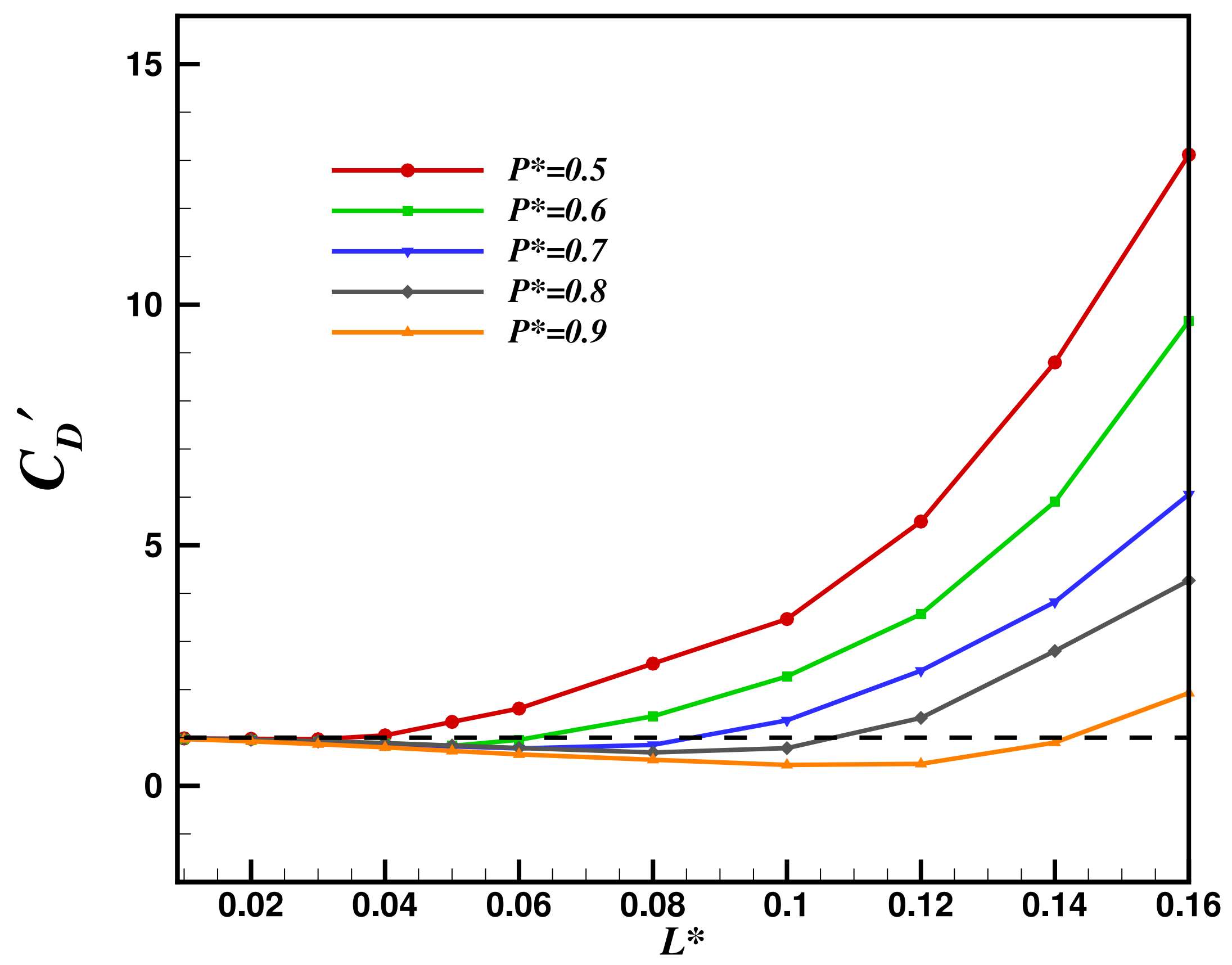




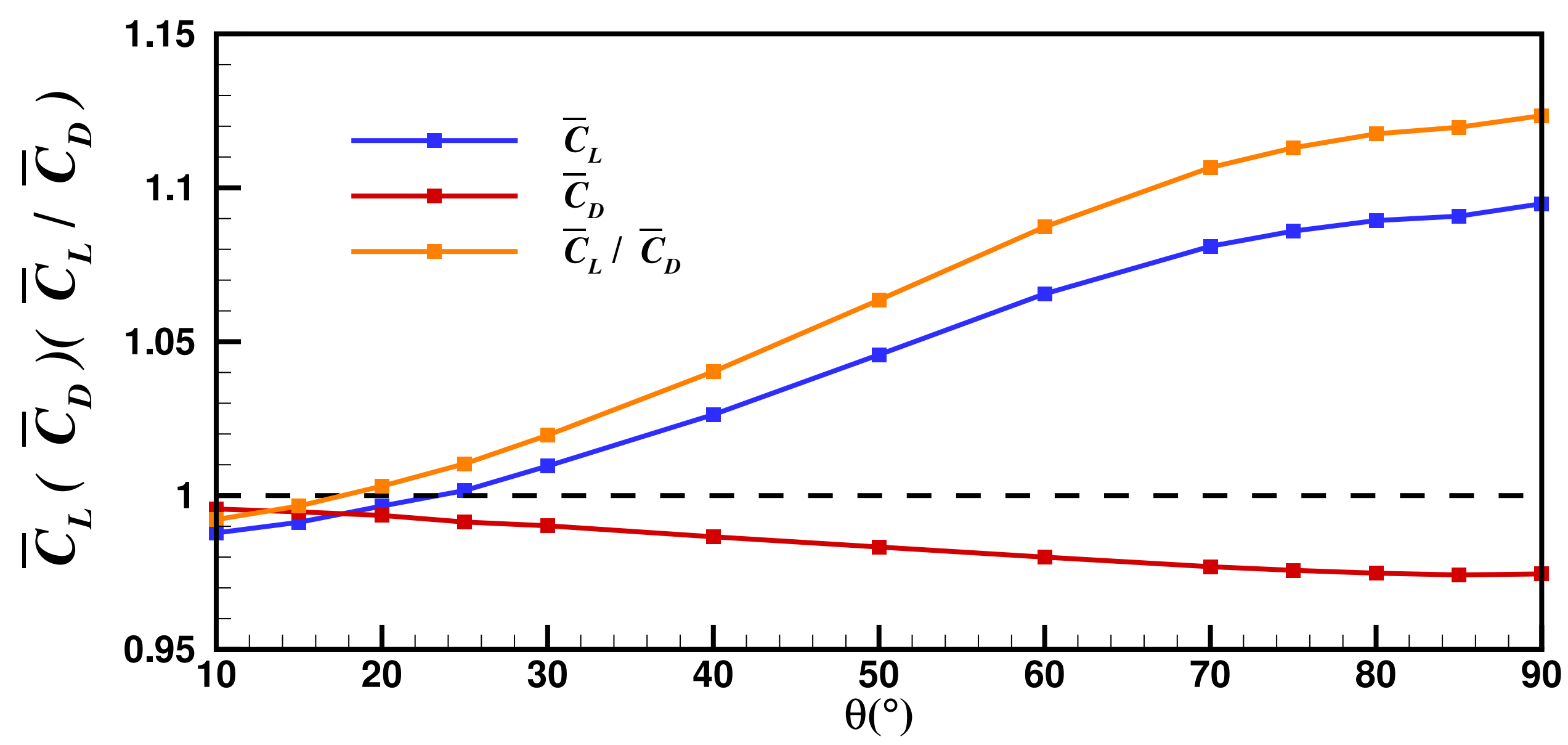




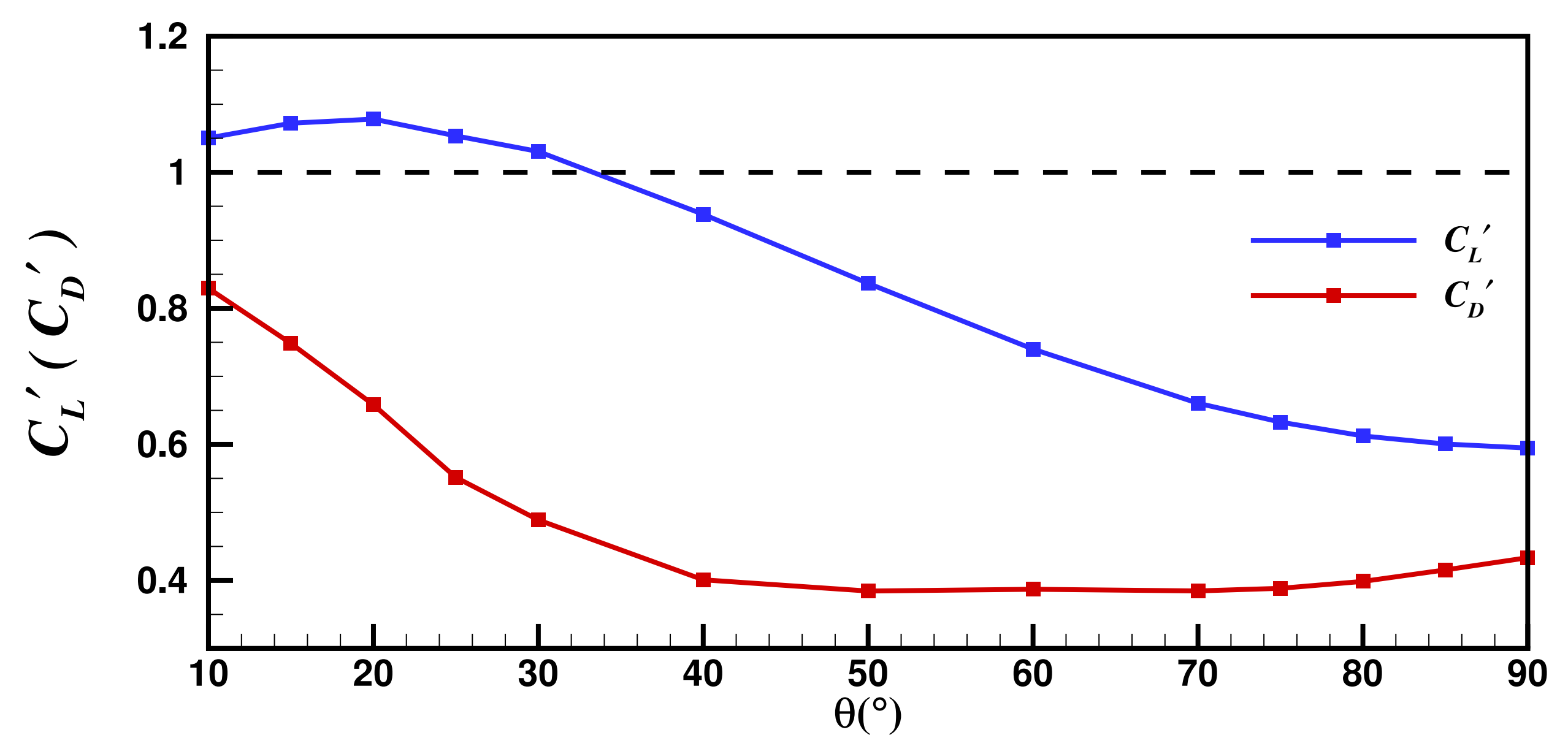




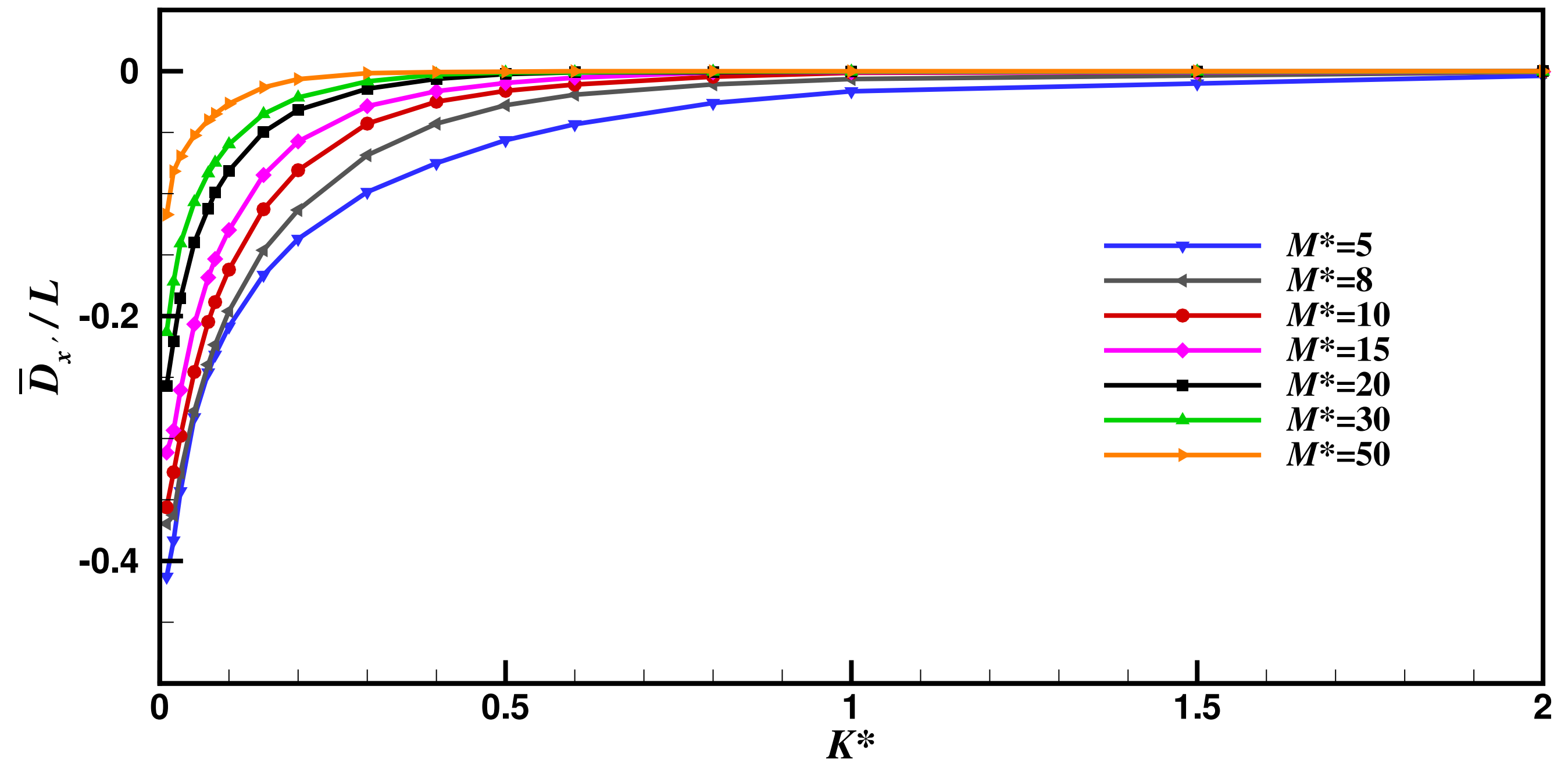




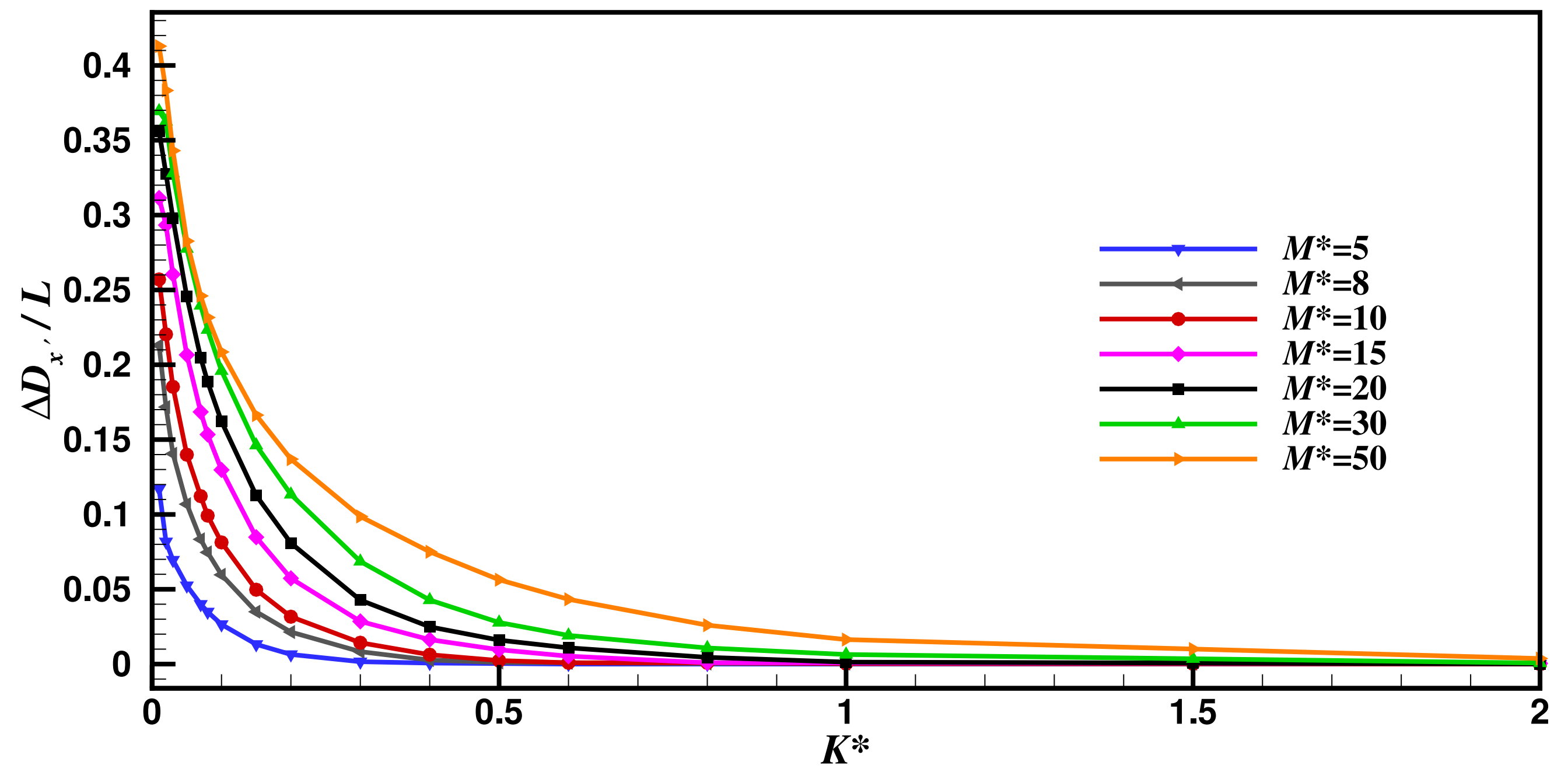




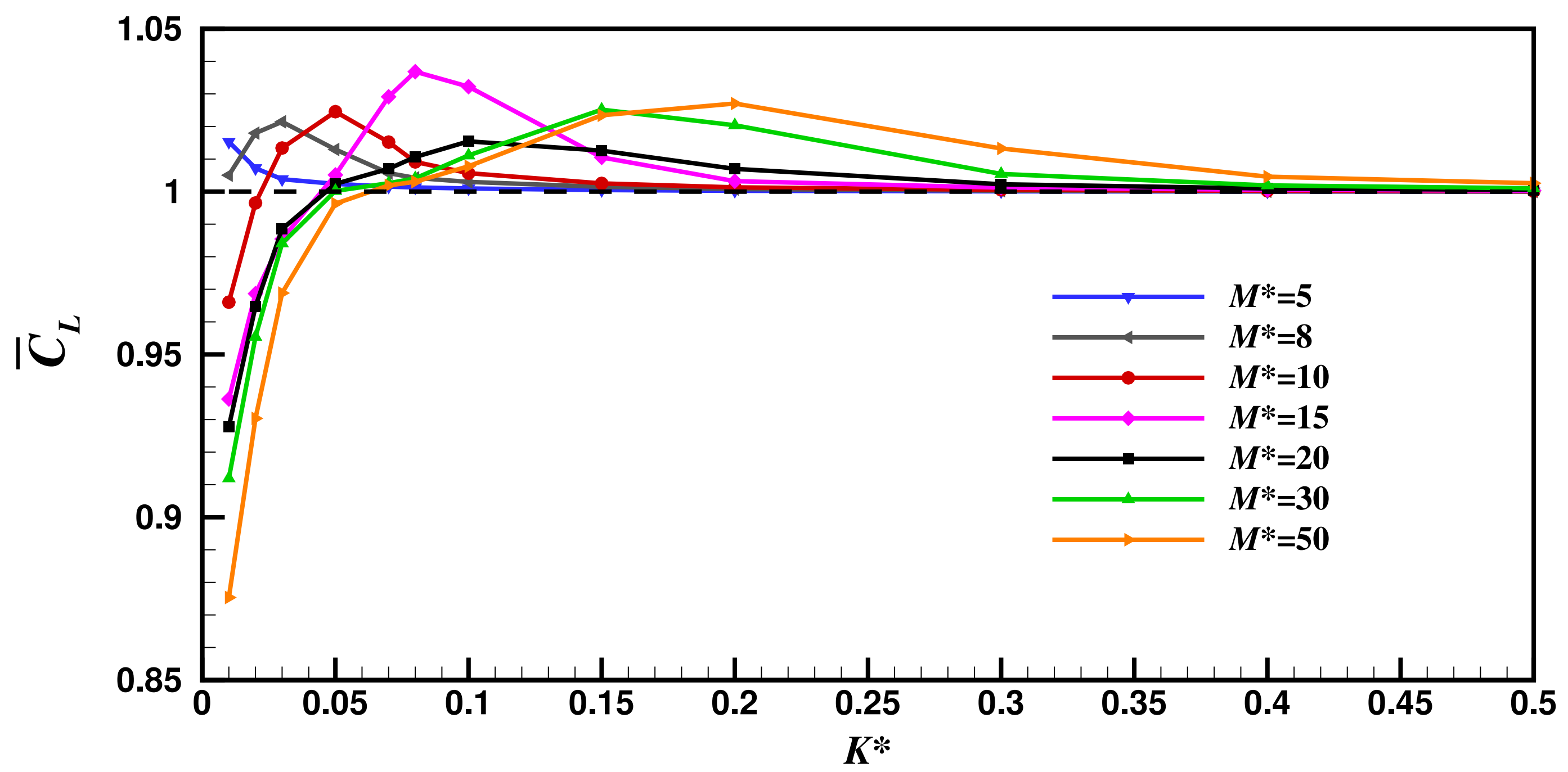




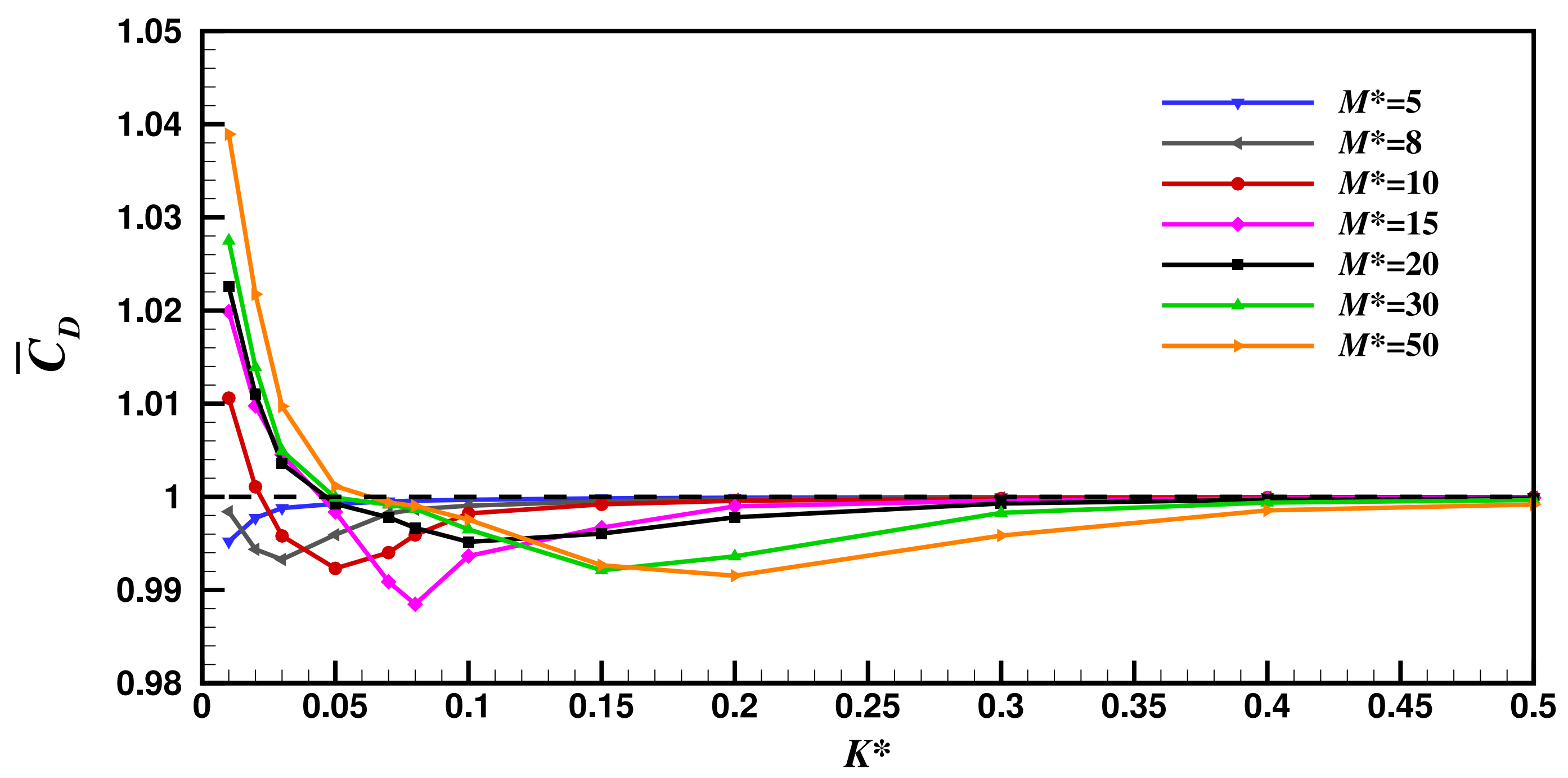




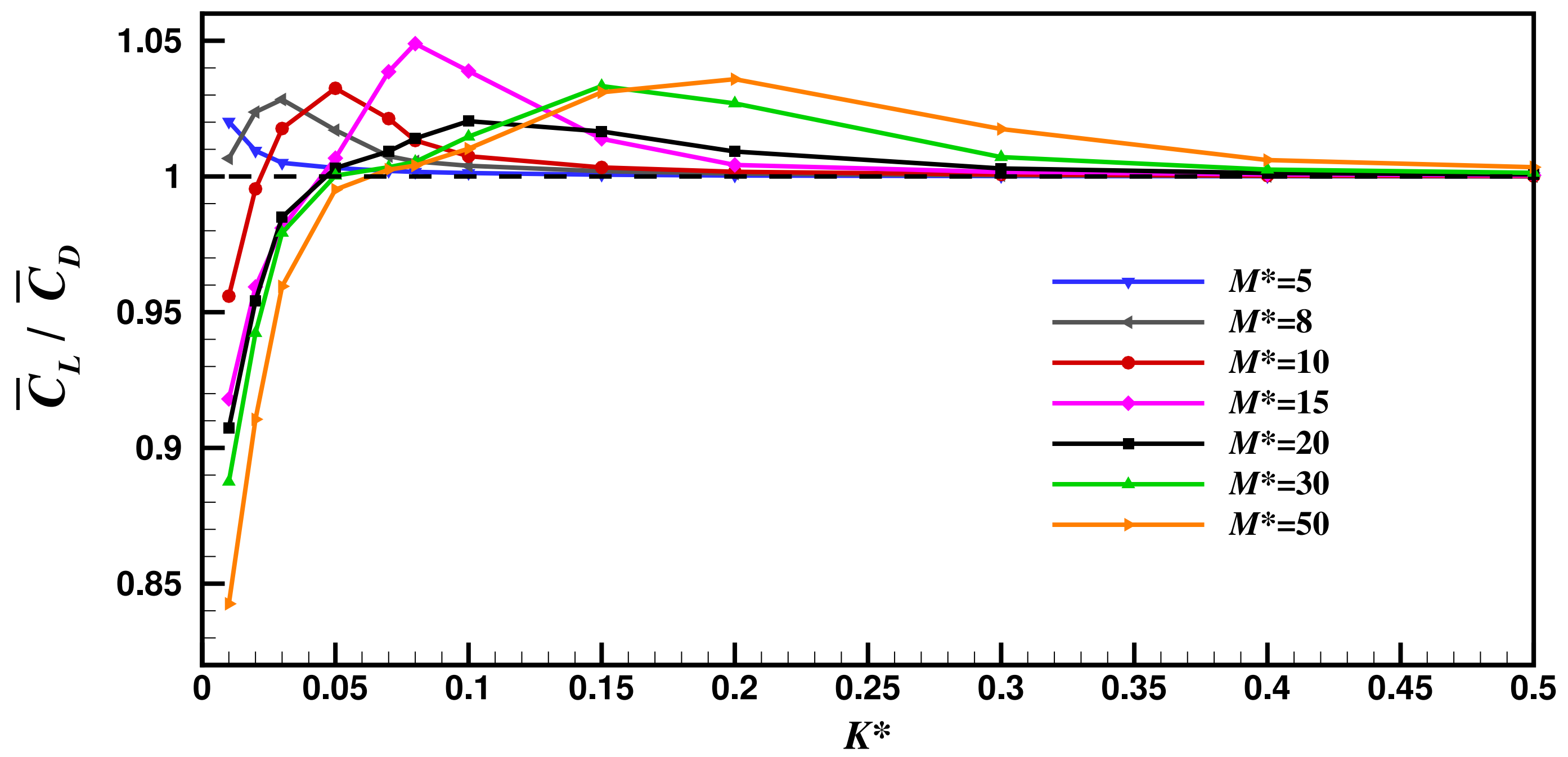




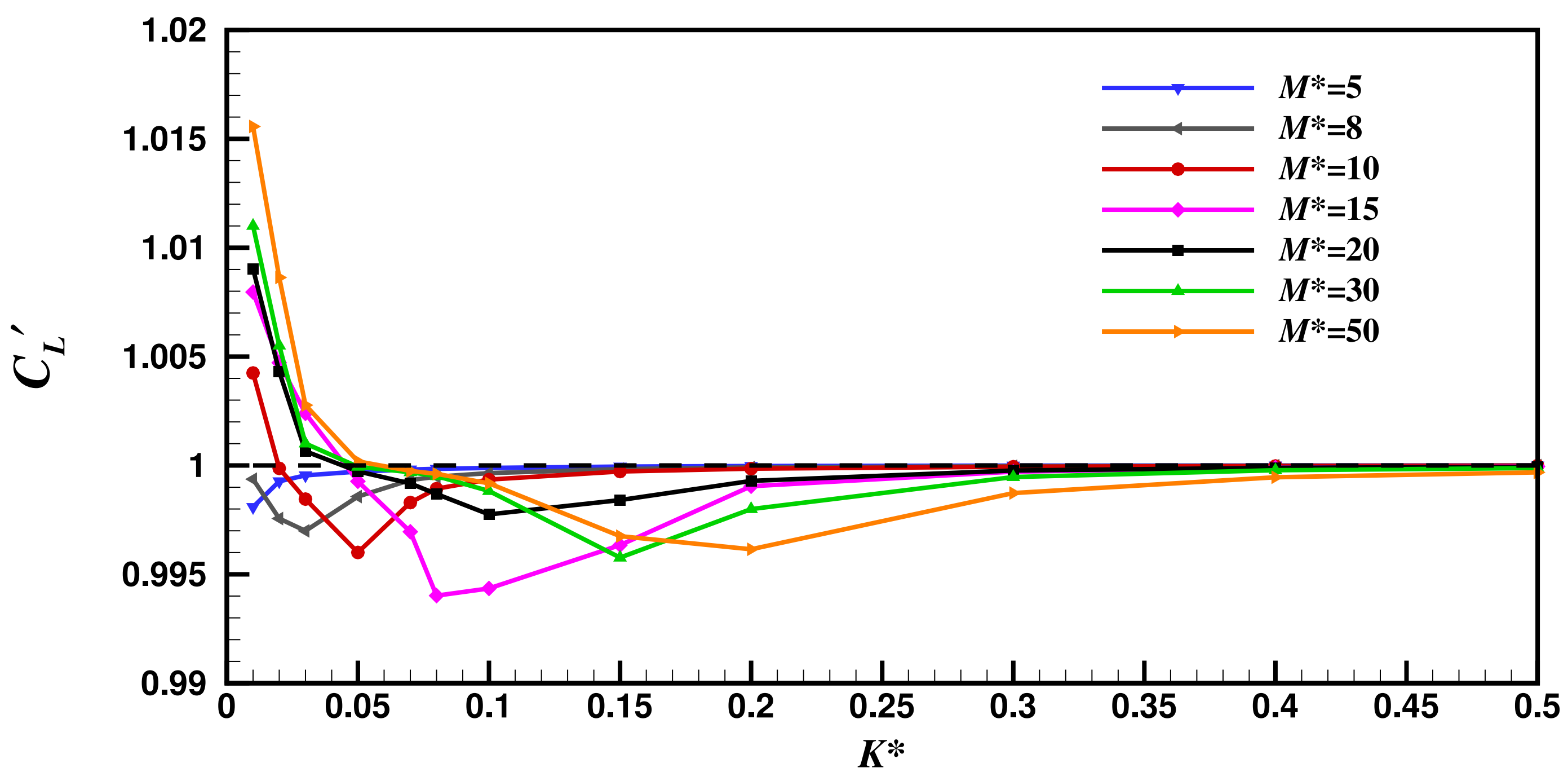




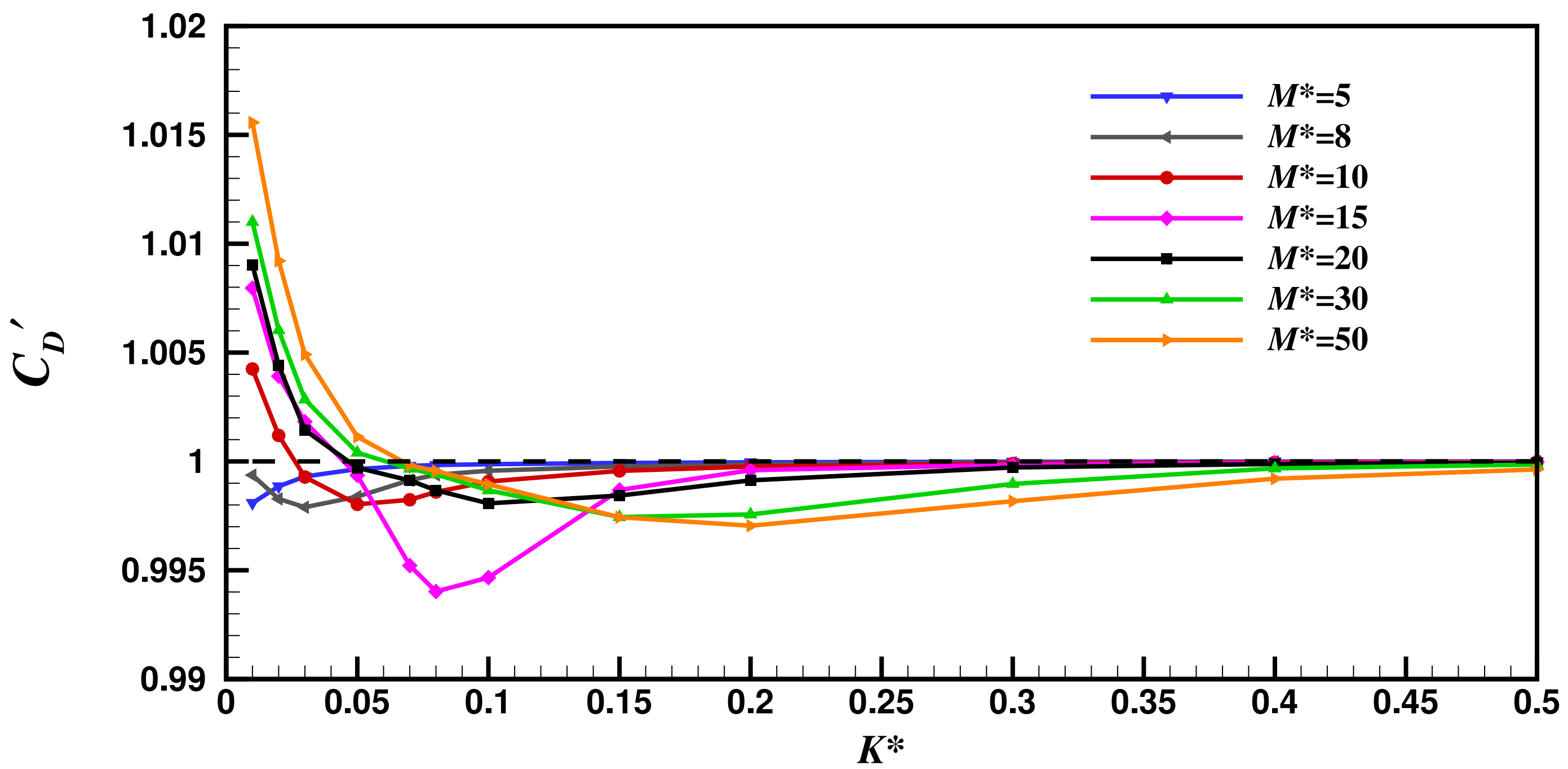




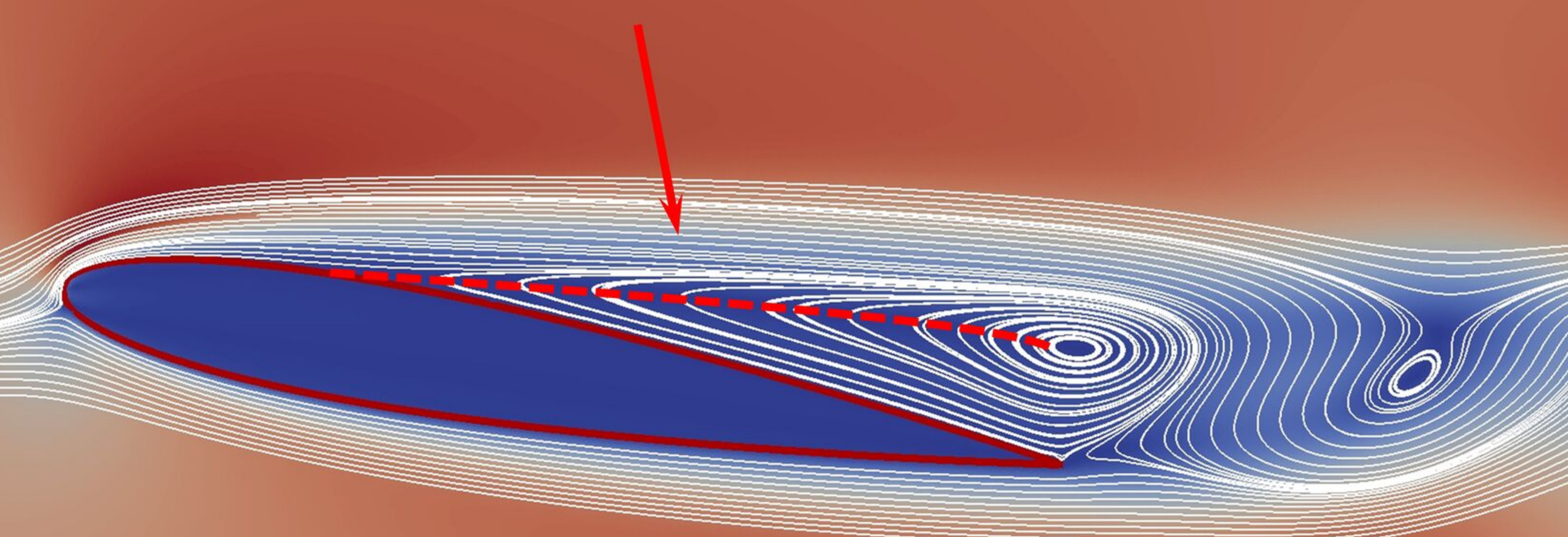




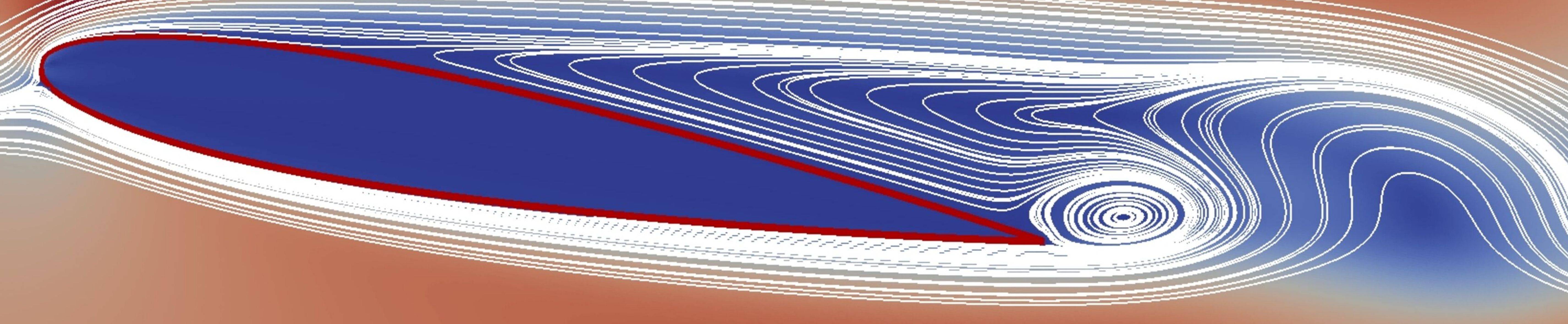




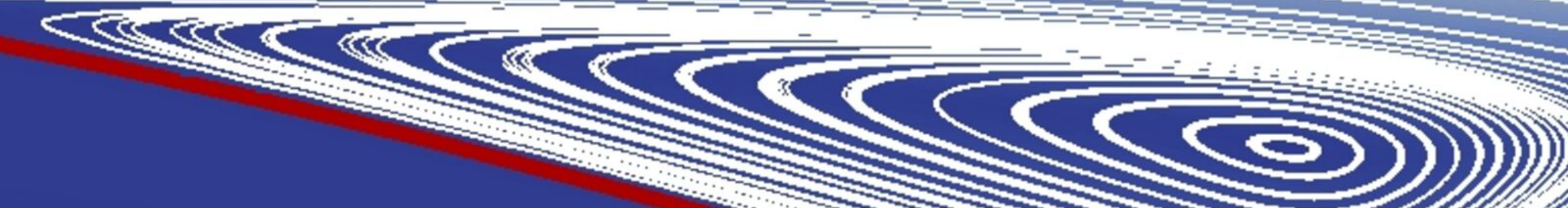

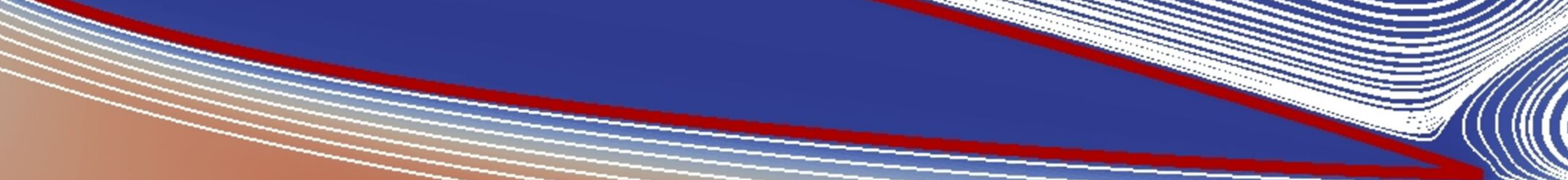

mon 


\title{
Velocity
}

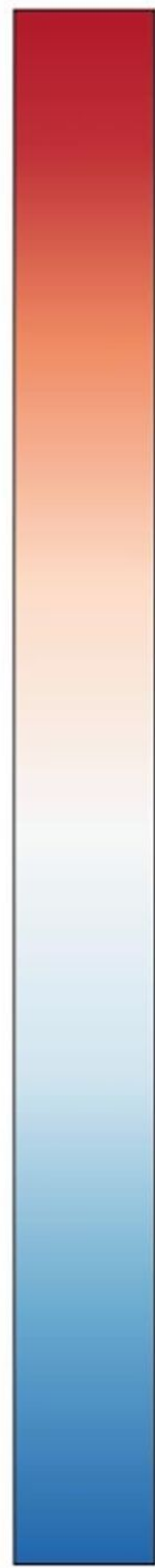

\author{
0.065
}

0.052

0.039

0.026

0.013

0.000 


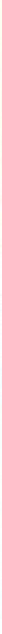




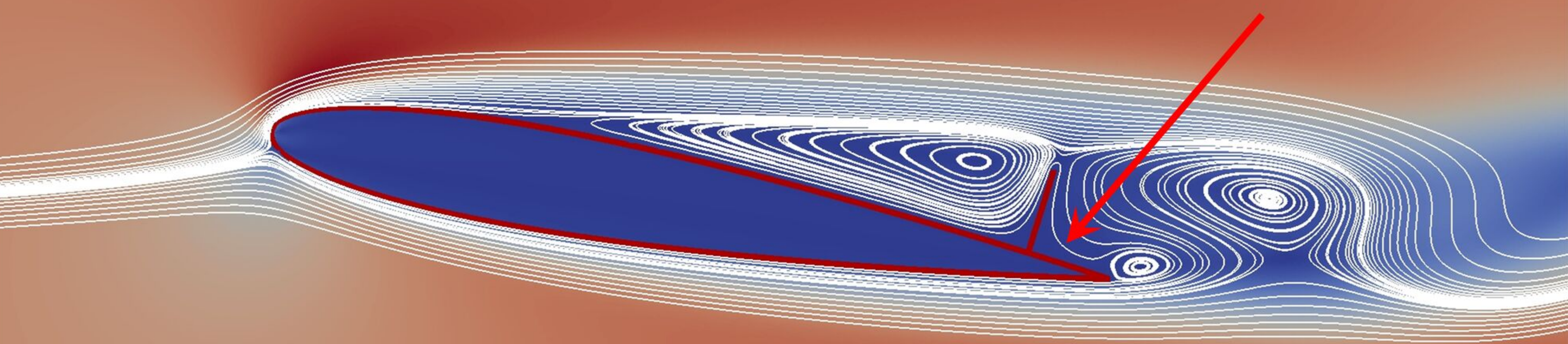




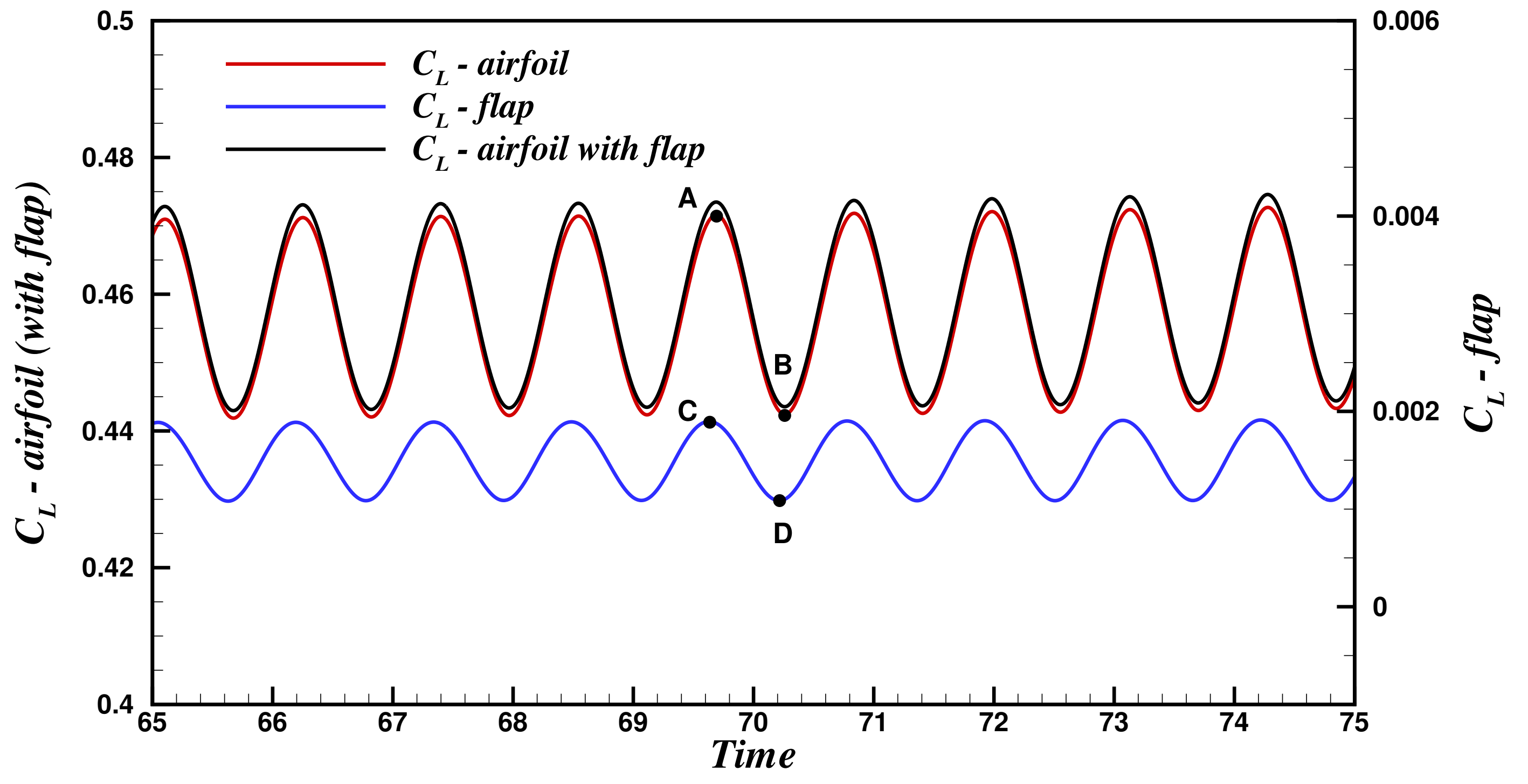




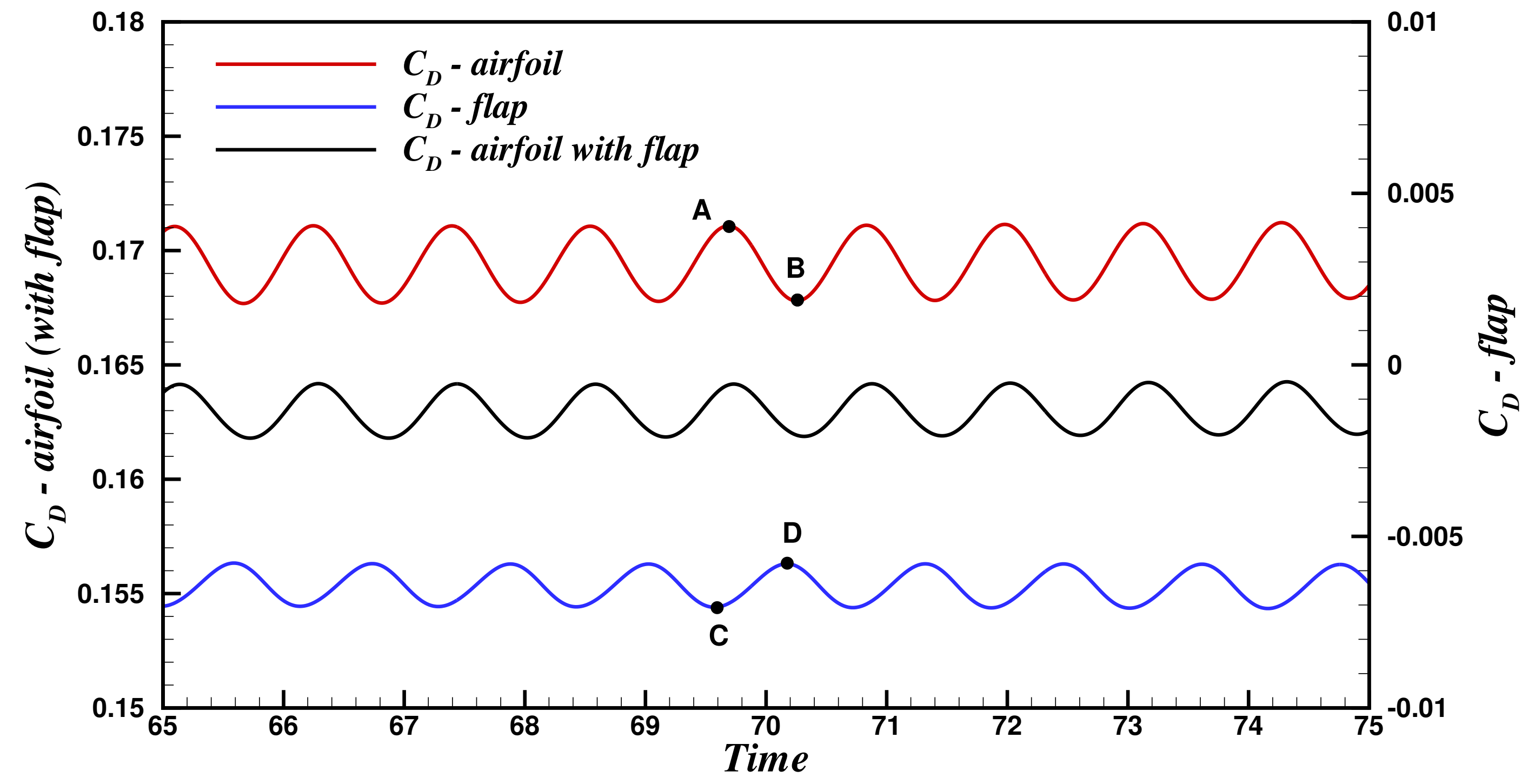


Velocity

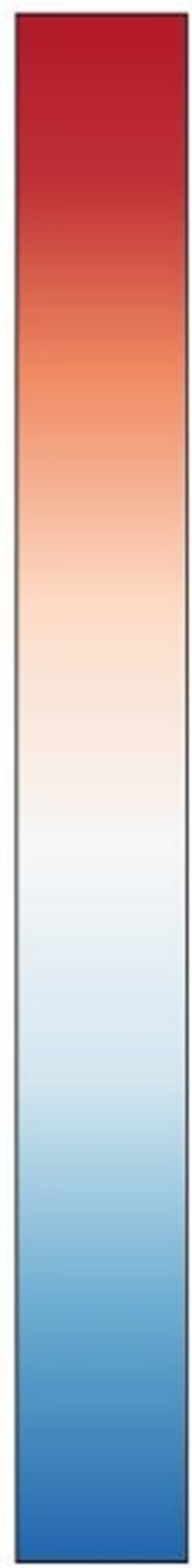

0.065

0.049

0.033

0.016

0.000 


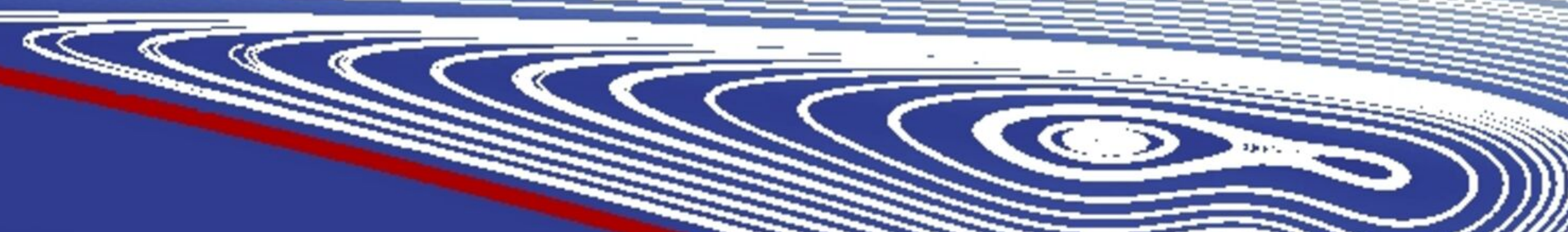

a

U 


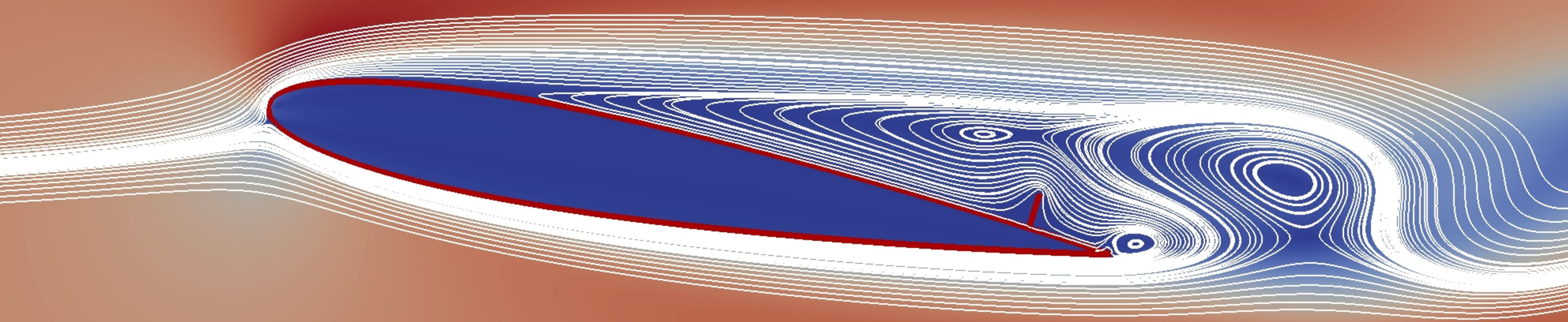




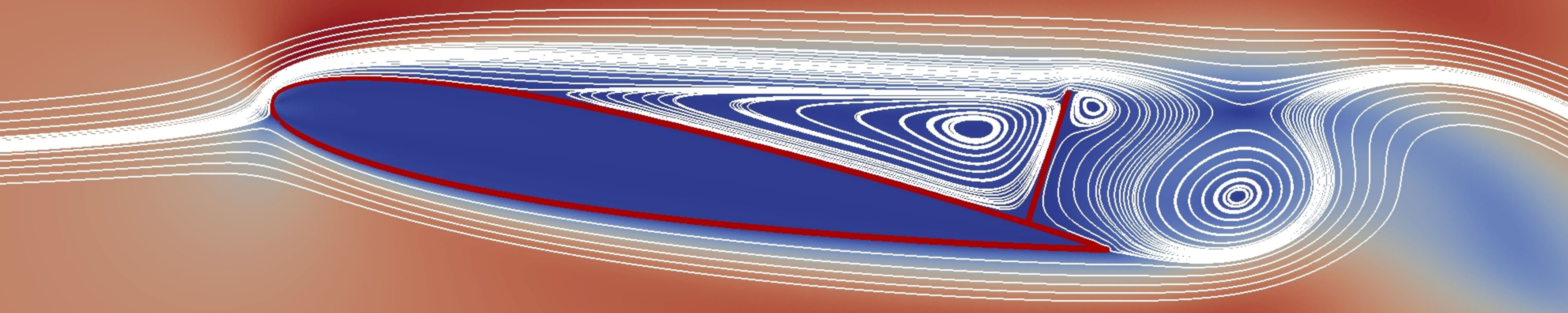




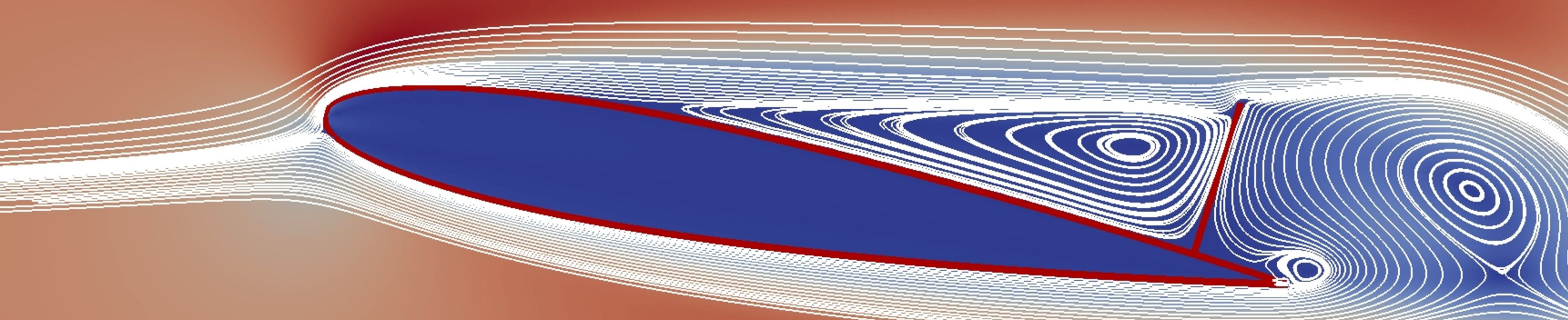




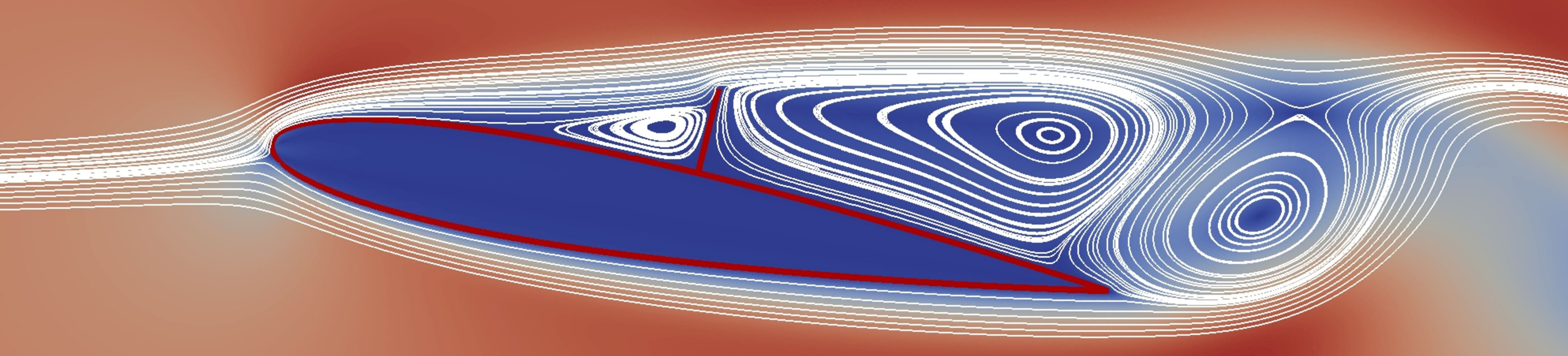




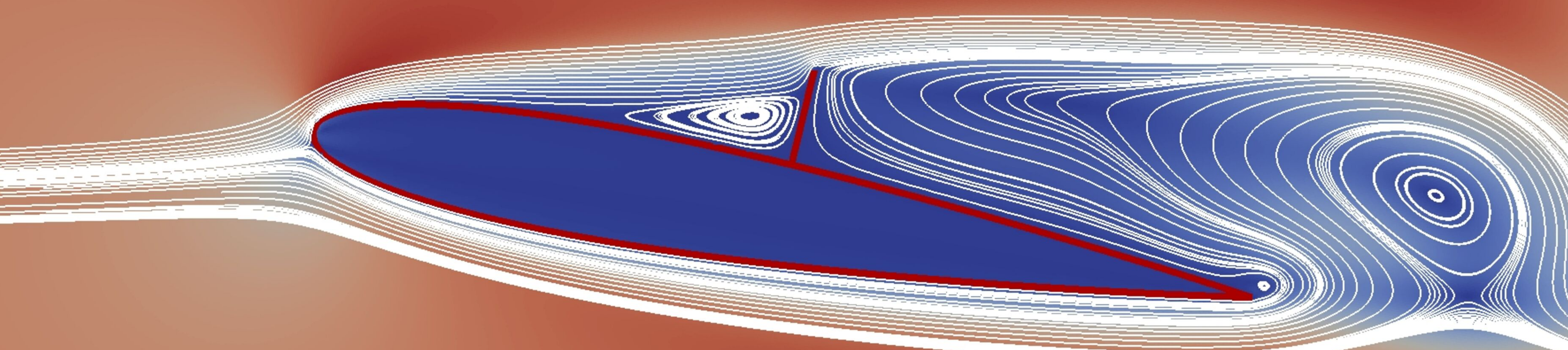


Velocity

0.065

0.056

0.046

0.037

0.028

0.019

0.009

0.000 
$U_{\infty}$ 



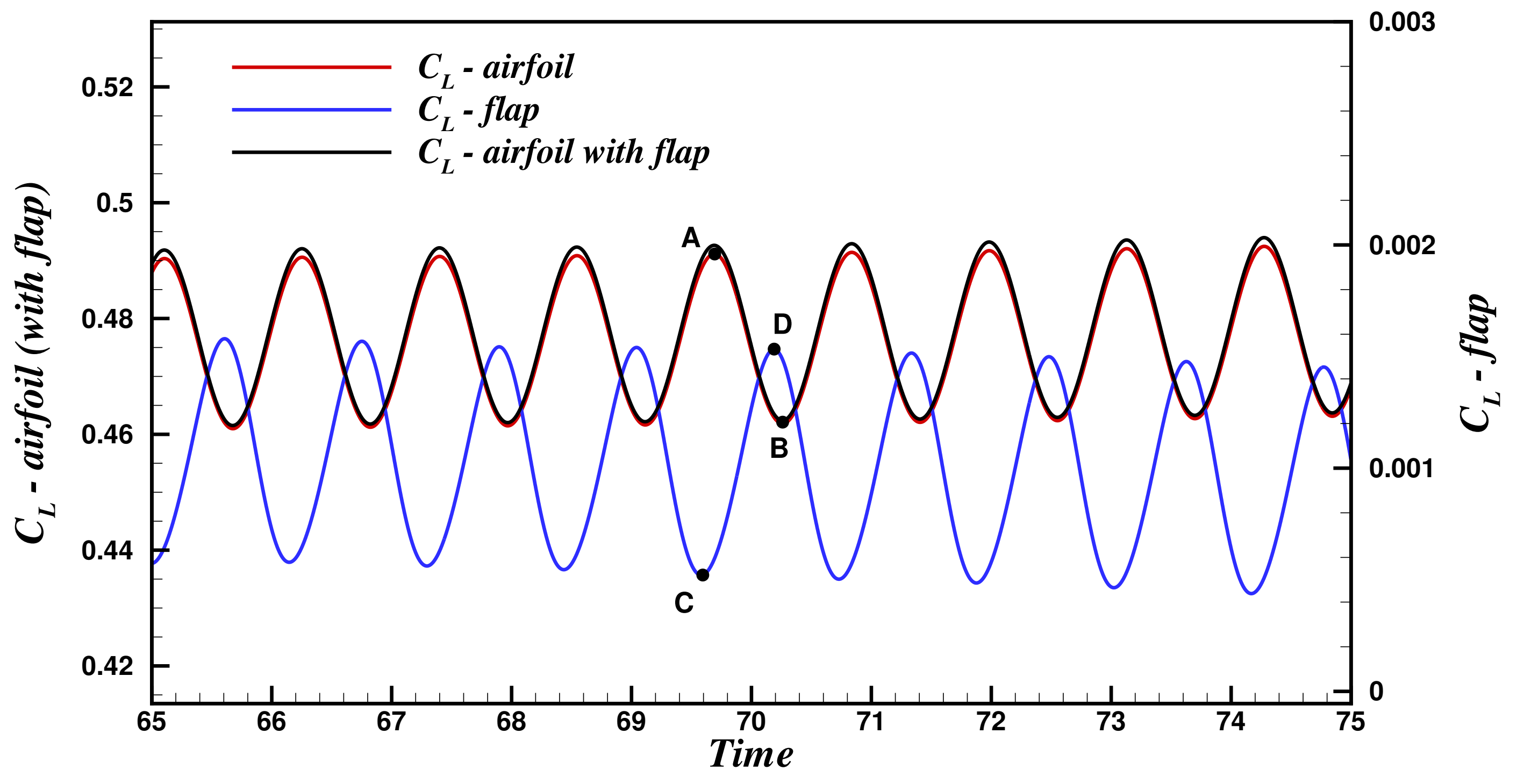




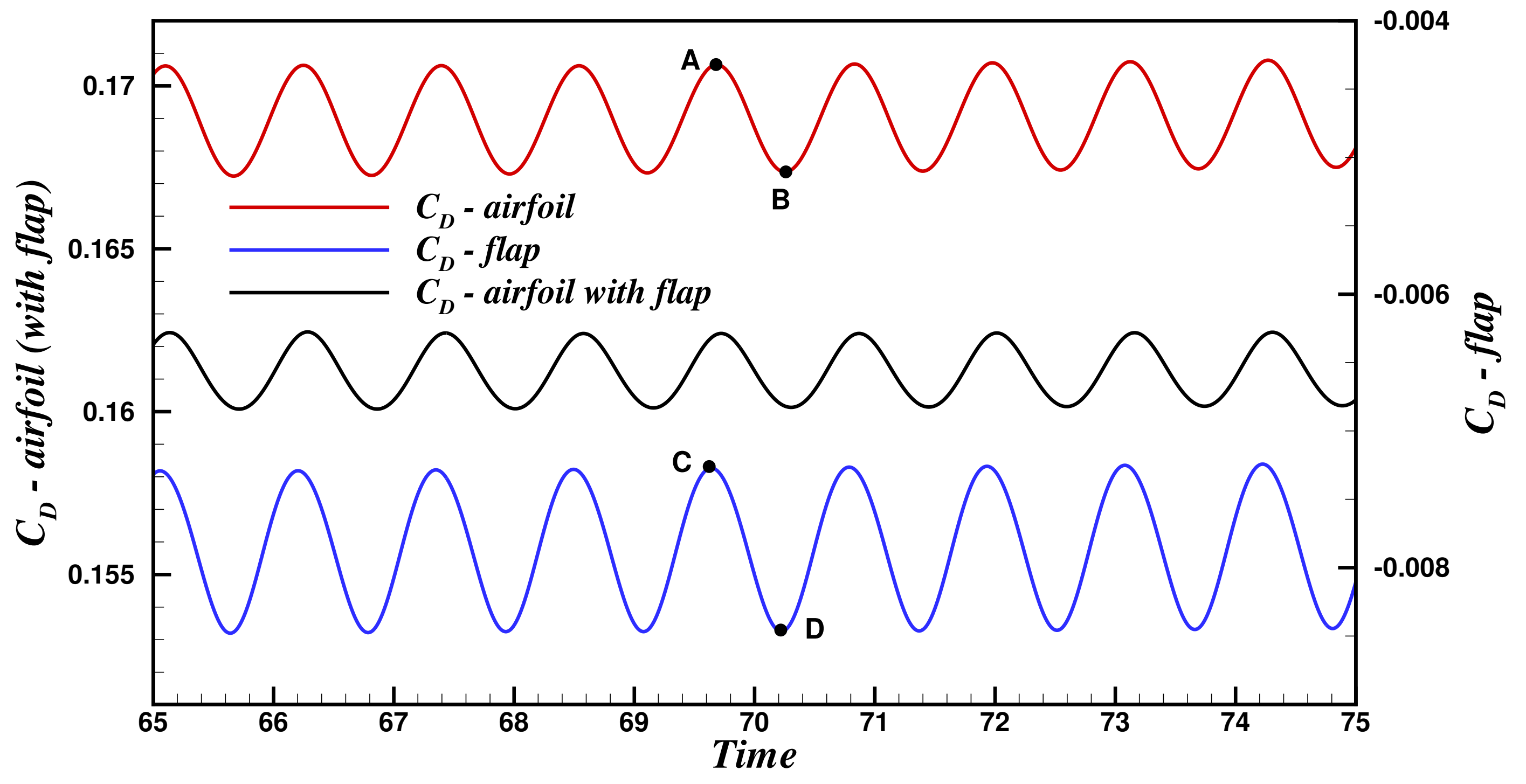




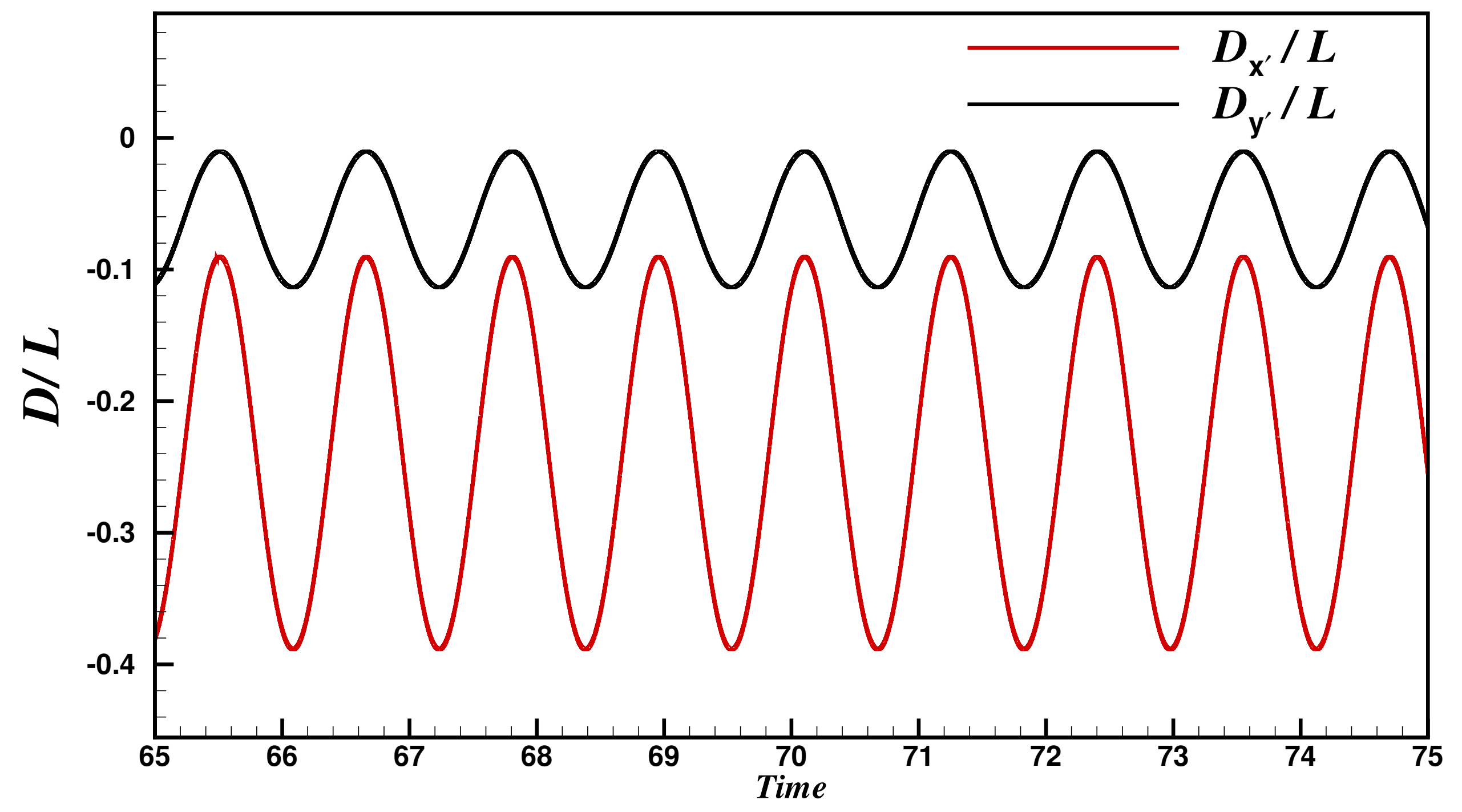


<smiles>C#CC(C)C</smiles> 
Velocity

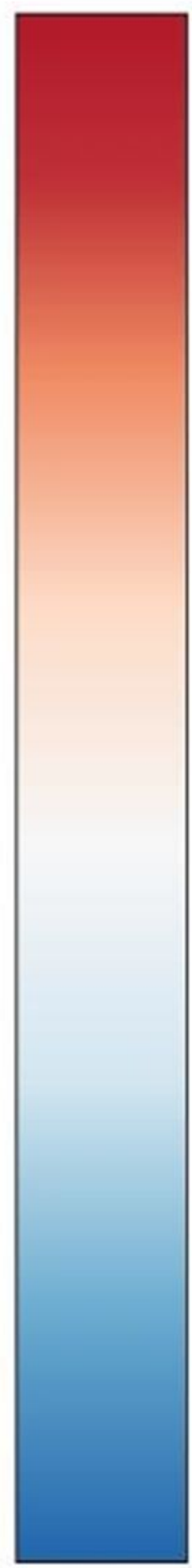

0.065

0.049

0.033

0.016

0.000 
( 


$$
\sim
$$




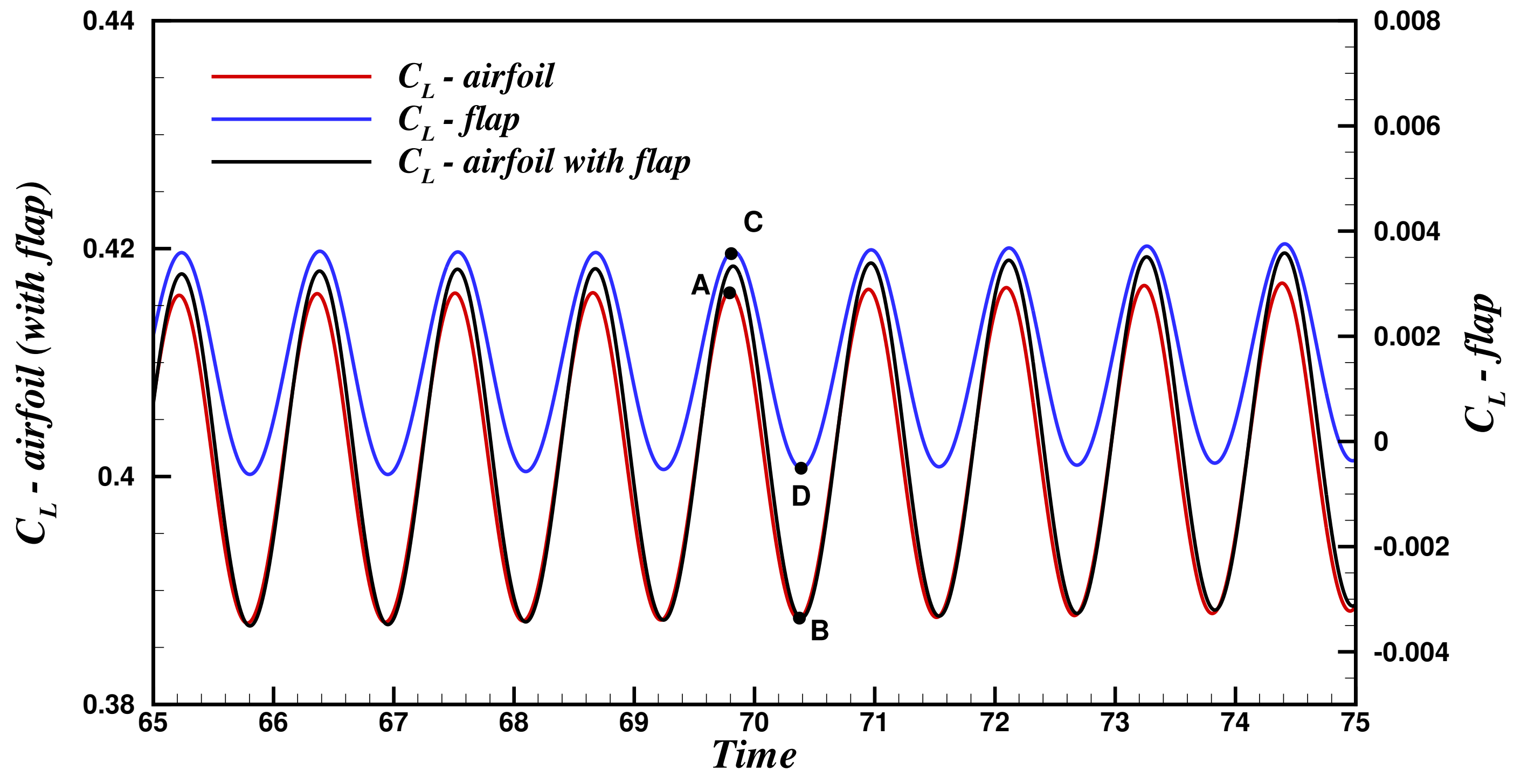




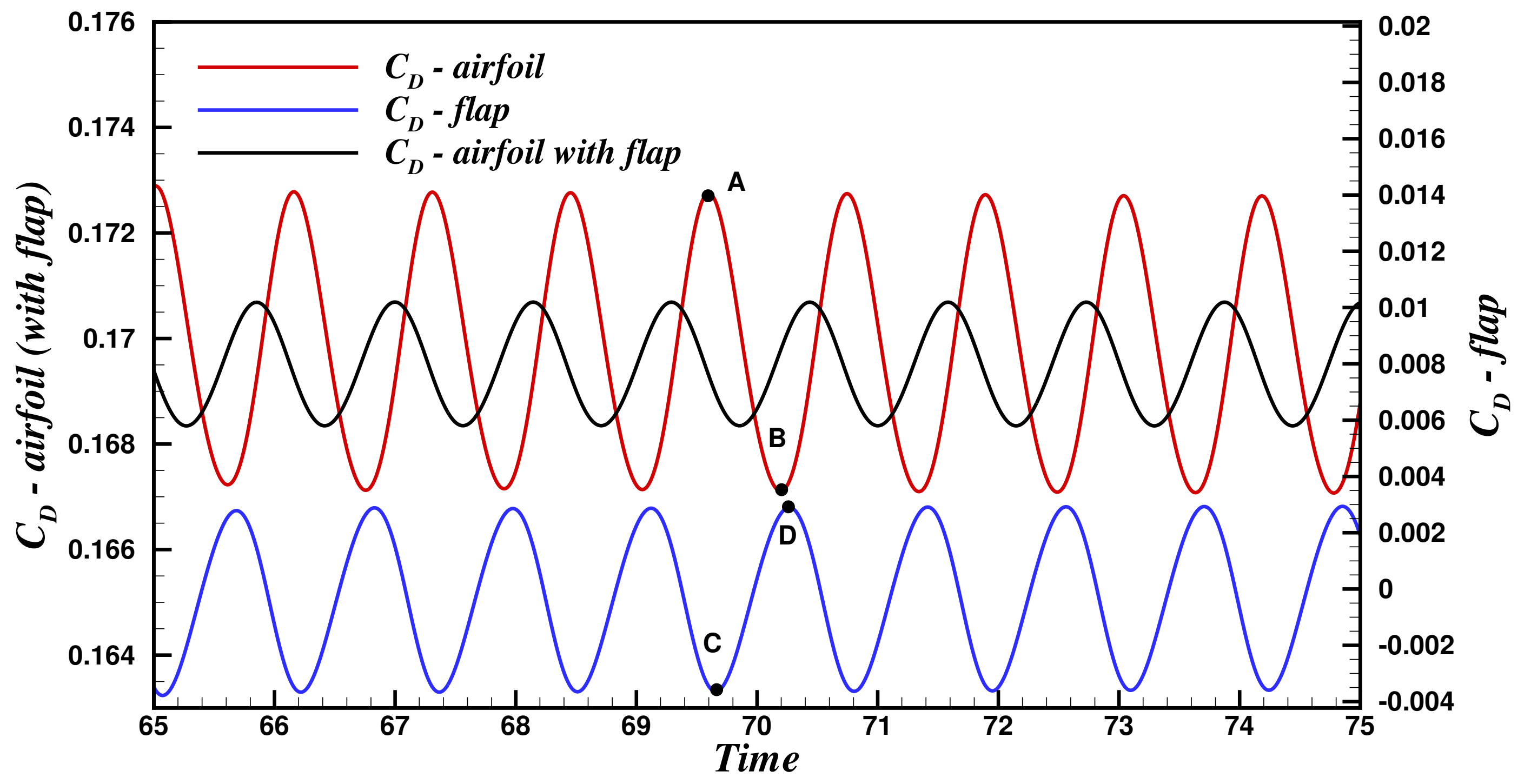




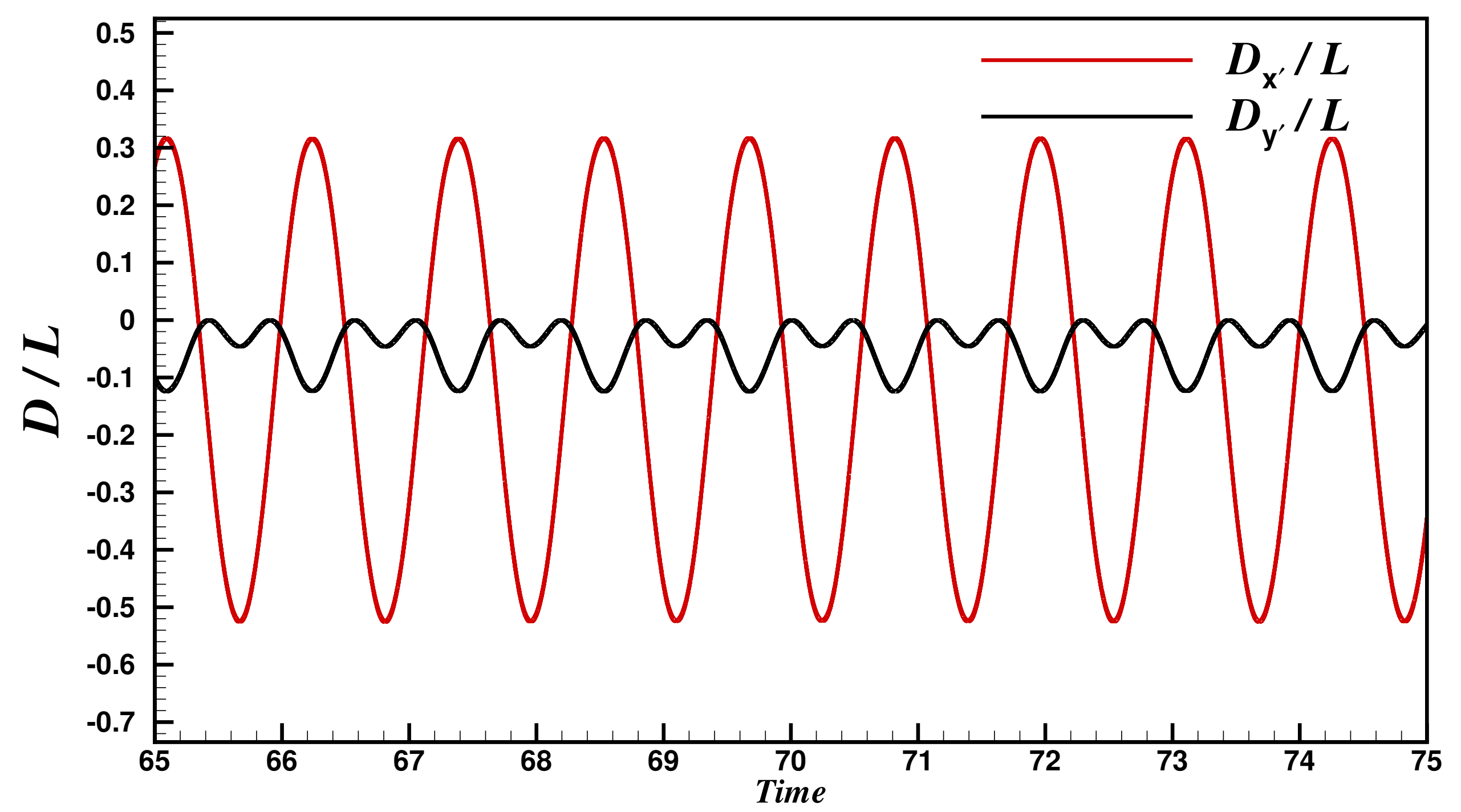



Velocity

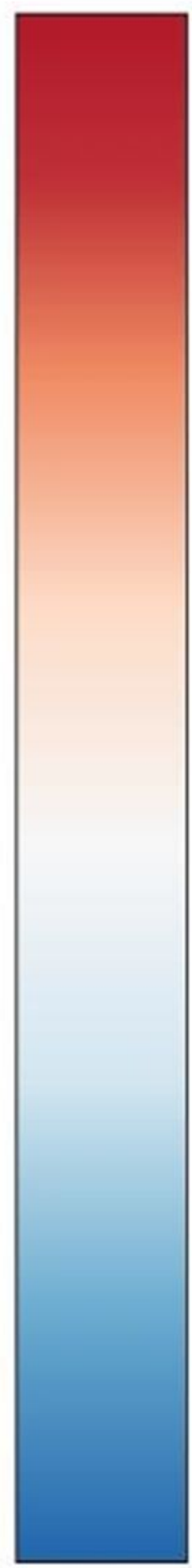

0.065

0.049

0.033

0.016

0.000 